GLASNIK MATEMATIČKI

Vol. 40(60)(2005), $347-384$

\title{
A SUBSHAPE SPECTRUM FOR COMPACTA
}

\author{
NikICA UGLEŠIĆ AND BRANKo ČERVAR \\ University of Split, Croatia
}

\begin{abstract}
A sequence of categories and functors between them are constructed. They form a subshape spectrum for compacta in the following sense: Each of these categories classifies compact ANR's just as the homotopy category does; the classification of compacta by the "finest" of these categories coincides with the shape type classification; moreover, the finest category contains a subcategory which is isomorphic to the shape category; there exists a functor of the shape category to each of these categories, as well as of a "finer" category to a "coarser" one; the functors commute according to the indices.

Further, a few applications of the "subshape spectrum theory" are demonstrated. It is shown that the $S^{*}$-equivalence (a uniformization of the Mardešić $S$-equivalence) and the $q^{*}$-equivalence (a uniformization of the Borsuk quasi-equivalence) admit the category characterizations within the subshape spectrum, and that the $q^{*}$-equivalence implies (but is not equivalent to) the $S^{*}$-equivalence.
\end{abstract}

\section{INTRODUCTION}

In the year 1968 the shape theory of (metrizable) compacta was founded by K. Borsuk [1]. The corresponding classification of compacta is generally coarser than the homotopy type classification, while on the subclass of locally nice spaces (compact ANR's) it coincides with the homotopy type classification. Since 1976 a few new classifications of compacta have been considered. For instance, K. Borsuk [2] introduced the relations of quasi-affinity and quasi-equivalence, while S. Mardešić [6] introduced the $S$-equivalence relation between compacta. All of them are shape type invariant relations. These classifications are strictly coarser than the shape type classification

2000 Mathematics Subject Classification. 55P55, 18A32.

Key words and phrases. Compactum, ANR, inverse sequence, limit, shape type, quasiequivalence, S-equivalence. 
$([2,5,3])$. Moreover, the quasi-equivalence and $S$-equivalence classifications coincide with the homotopy type classification on compact ANR's. However, the mentioned relations, being defined only on the class of objects, were not supported by appropriate with them associated theories. In other words, it was not clear whether these relations are categorical. Furthermore, if such an equivalence relation admits a category characterization, there should exist a functor relating the shape category and the new category.

On this line the first named author studied in [9] the Borsuk quasiequivalence $\stackrel{q}{\simeq}$ and quasi-affinity $\stackrel{q}{\leftrightarrow}$. He constructed a certain category and an appropriate "quasi-homotopy" relation $\stackrel{q}{\simeq}$ on its morphism sets such that

$$
\begin{aligned}
& X \stackrel{q}{\simeq} Y \Leftrightarrow(\exists u: X \rightarrow Y)(\exists v: Y \rightarrow X) v u \stackrel{q}{\simeq} 1_{X} \wedge u v \stackrel{q}{\simeq} 1_{Y} ; \\
& X \stackrel{q}{\leftrightarrow} Y \Leftrightarrow\left(\exists u, u^{\prime}: X \rightarrow Y\right)\left(\exists v, v^{\prime}: Y \rightarrow X\right) v u \stackrel{q}{\simeq} 1_{X} \wedge u^{\prime} v^{\prime} \stackrel{q}{\simeq} 1_{Y} ;
\end{aligned}
$$

However, the morphism equivalence relation $\stackrel{q}{\simeq}$ is not compatible (from the left) with the category composition, so there is no corresponding quotient category. Nevertheless, one can slightly strengthen the Borsuk relation $\stackrel{q}{\simeq}$ $(\stackrel{q}{\leftrightarrow})$ up to the new equivalence relation $\stackrel{\bar{q}}{\simeq}, \bar{q}$-equivalence $(\stackrel{\bar{q}}{\leftrightarrow}, \bar{q}$-affinity) on compacta, which admits a characterization in terms of a category isomorphism (domination), i.e. there exists a (quotient) category $\mathcal{Q}$ such that

$$
\begin{aligned}
& X \stackrel{\bar{q}}{\simeq} Y \Leftrightarrow X \cong Y \text { in } \mathcal{Q} ; \\
& X \stackrel{\bar{q}}{\leftrightarrow} Y \Leftrightarrow X \leftrightarrow Y \text { in } \mathcal{Q} \text { (i.e. } X \leq Y \wedge Y \leq X \text { in } \mathcal{Q} \text { ). }
\end{aligned}
$$

There also exist two functors $Q: H c \mathcal{M} \rightarrow \mathcal{Q}$ and $\Gamma: \mathcal{S} h \rightarrow \mathcal{Q}$ such that $\Gamma S=Q$, where $S: H c \mathcal{M} \rightarrow \mathcal{S} h$ is the ordinary shape functor. (HcM $\mathcal{M}$ and $\mathcal{S} h$ are the homotopy category of compacta and the shape category of compacta, respectively.) Furthermore, there exists a "uniformization" $\stackrel{q^{*}}{\simeq}$ ( $q^{*}$-equivalence) of $\stackrel{\bar{q}}{\simeq}$ which admits a quite similar category characterization.

With a similar purpose Mardešić and Uglešić [7] studied the $S^{*}$-equivalence, which is a "uniformization" of the Mardešić $S$-equivalence. They constructed a certain category $\mathcal{S}^{*}$ on compacta such that

$$
S^{*}(X)=S^{*}(Y) \Leftrightarrow X \cong Y \text { in } \mathcal{S}^{*} .
$$

They also obtained the appropriate functor $S^{*}: \mathcal{S} h \rightarrow \mathcal{S}^{*}$. We should mention that the $S$-equivalence as well as the $S^{*}$-equivalence of compacta is a rather useful notion. For instance, some important shape invariant classes of compacta (continua, movable compacta, $n$-shape connected compacta, FANR's, compacta having $F d \leq n, \ldots)$ are also $S$ - and $S^{*}$-invariant classes. Furthermore, it is a well known fact that the fibres of a shape fibration over a continuum may have different shape. However, all of them are mutually $S$ and $S^{*}$-equivalent. 
In this paper we have first constructed a certain "graded" family of categories on compacta $\mathcal{S}(n), n \in \mathbb{N} \cup\{\omega\}$ (as well as the corresponding sequence category $\mathcal{S}(\mathbb{N})$ ), endowed with an appropriate family of functors, which commute according to the indices, such that they may represent a subshape spectrum for compacta in the following sense:

Each of these categories classifies compact ANR's as the homotopy category does. The "finest" of these categories classifies compacta as the shape category does. Moreover, it contains a subcategory which is isomorphic to the shape category. Further, there exists a functor of the shape category to each of these categories, as well as of a "finer" category to a "coarser" one; these functors commute according to the indices. (The category theory preliminaries are taken from [4].)

After the theoretical part, we demonstrate a few applications of the obtained "subshape spectrum theory". We have proved that the $S^{*}$-equivalence and $q^{*}$-equivalence admit category characterizations within the subshape spectrum, and that the $q^{*}$-equivalence strictly implies the $S^{*}$-equivalence. Further, the homotopy type classification of compact ANR's coincides with the isomorphism classification in the category $\mathcal{S}(1)$, while the shape type classification of FANR's reduces to the isomorphism classification in a subcategory of $\mathcal{S}(2)$.

Since we consider the relations which classify compacta, our main objects are the inverse sequences of compact ANR's [8]. The smallest building material for a morphism is a 1-ladder, which imitates a mapping of inverse sequences restricted to a finite piece. By fitting together finitely many of them one obtains an $n$-ladder, $n \in \mathbb{N}$. The infinite case $n=\omega$ is also allowed. A countable collection of such $n$-ladders, subjected to some conditions, is called an $n$-hyperladder. The $n$-hyperladders are organized into a category $\underline{\mathcal{L}}(n)$ (on the inverse sequences). Such a category admits a natural equivalence (homotopy) relation $\simeq$, which yields the quotient category $\underline{\mathcal{S}}(n)$. These categories $\underline{\mathcal{S}}(n), n \in \mathbb{N} \cup\{\omega\}$, and the sequence category $\underline{\mathcal{S}}(\mathbb{N})$ form the mentioned "graded" family of categories having inverse sequences as objects. Clearly, there also exists the corresponding "graded" family of categories $\mathcal{S}(n), \mathcal{S}(\mathbb{N})$ on compacta. The appropriate functors arise almost naturally. We also consider some of the useful subcategories and the corresponding functors.

\section{The SUbShaPe CATEGORIES}

First of all, recall the shape category of compacta (compact metrizable spaces $X, Y, \ldots),[8]$. Let $\underline{\mathcal{S}}$ denote the category having all compact ANR inverse sequences $\left.\boldsymbol{X}=\left(X_{i},\left[p_{i i^{\prime}}\right], \mathbb{N}\right]\right), \boldsymbol{Y}=\left(Y_{j},\left[q_{j j^{\prime}}\right], \mathbb{N}\right), \ldots$ as objects (bonding maps are the homotopy classes of mappings), while

$$
\underline{\mathcal{S}}(\boldsymbol{X}, \boldsymbol{Y})=\left\{\boldsymbol{f} \mid \boldsymbol{f}=\left(f,\left[f_{j}\right]\right): \boldsymbol{X} \rightarrow \boldsymbol{Y}\right\},
$$


where $f: \mathbb{N} \rightarrow \mathbb{N}$ is a function (the index function), and $f_{j}: X_{f(j)} \rightarrow Y_{j}$, $j \in \mathbb{N}$, are mappings satisfying the following condition:

$$
\left(\forall j \leq j^{\prime}\right)\left(\exists i \geq f(j), f\left(j^{\prime}\right)\right)\left[f_{j}\right]\left[p_{f(j) i}\right]=\left[q_{j j^{\prime}}\right]\left[f_{j^{\prime}}\right]\left[p_{f\left(j^{\prime}\right) i}\right] .
$$

The identity morphism $\mathbf{1}_{\boldsymbol{X}}=\left(1_{\mathbb{N}},\left[1_{X_{i}}\right]\right)$, and the composition of an $\boldsymbol{f}: \boldsymbol{X} \rightarrow$ $\boldsymbol{Y}$ with a $\boldsymbol{g}=\left(g,\left[g_{k}\right]\right): \boldsymbol{Y} \rightarrow \boldsymbol{Z}$ is defined by

$$
\boldsymbol{g} \boldsymbol{f}=\left(f g,\left[g_{k} f_{g(k)}\right]\right): \boldsymbol{X} \rightarrow \boldsymbol{Z} .
$$

A morphism $\boldsymbol{f}: \boldsymbol{X} \rightarrow \boldsymbol{Y}$ is said to be special provided $f$ is increasing and

$$
\left(\forall j \leq j^{\prime}\right)\left[f_{j}\right]\left[p_{f(j) f\left(j^{\prime}\right)}\right]=\left[q_{j j^{\prime}}\right]\left[f_{j^{\prime}}\right] .
$$

An $\boldsymbol{f}: \boldsymbol{X} \rightarrow \boldsymbol{Y}$ is said to be homotopic to a morphism $\boldsymbol{f}^{\prime}=\left(f^{\prime},\left[f_{j}^{\prime}\right]\right): \boldsymbol{X} \rightarrow$ $\boldsymbol{Y}$, denoted by $\boldsymbol{f} \simeq \boldsymbol{f}^{\prime}$, provided

$$
(\forall j \in \mathbb{N})\left(\exists i \geq f(j), f^{\prime}(j)\right)\left[f_{j}\right]\left[p_{f(j) i}\right]=\left[f_{j}^{\prime}\right]\left[p_{f^{\prime}(j) i}\right] .
$$

The homotopy relation $\simeq$ is a natural equivalence relation on the category $\underline{\mathcal{S}}$. The homotopv class of an $\boldsymbol{f}$ is denoted by $[\boldsymbol{f}]$ or by $\boldsymbol{f}$. The corresponding quotient category $\underline{\mathcal{S}} \simeq \simeq$ is denoted by $\underline{\mathcal{S}} h$. It realizes the shape category $\mathcal{S} h$ of compacta, i.e.

$$
\mathcal{S} h(X, Y) \approx \underline{\mathcal{S}} h(\boldsymbol{X}, \boldsymbol{Y})=\underline{\mathcal{S}}(\boldsymbol{X}, \boldsymbol{Y}) / \simeq,
$$

where $\boldsymbol{X}, \boldsymbol{Y}$ are associated with $X, Y$ respectively, such that $\lim \boldsymbol{X}=X$ and $\lim \boldsymbol{Y}=Y$. Notice that every shape morphism (homotopy class) $\underline{\boldsymbol{f}}$ admits a special representative.

Finally, there is a functor $S: H c \mathcal{M} \rightarrow \mathcal{S} h$ (the shape functor), which keeps the objects fixed, where $H c \mathcal{M}$ is the standard homotopy category of compacta. (By choosing a unique $\boldsymbol{X}$ for a given $X$, one also obtains a functor $\underline{S}: H c \mathcal{M} \rightarrow \underline{\mathcal{S}} h$.

Let $\boldsymbol{X}, \underline{\boldsymbol{Y}} \in \mathrm{Ob} \underline{\mathcal{S}}$. Let $\boldsymbol{J}(1)$ be the set of all pairs $\boldsymbol{j}^{1}=\left(j_{1}, j_{2}\right)$, where $j_{1}, j_{2} \in \mathbb{N}, j_{1}<j_{2}$. A 1-ladder $\boldsymbol{f}_{\boldsymbol{j}^{1}}=\left(f,\left[f_{j}\right]\right)$ of $\boldsymbol{X}$ to $\boldsymbol{Y}$ over $\boldsymbol{j}^{1} \in \boldsymbol{J}(1)$, denoted by $\boldsymbol{f}_{\boldsymbol{j}^{1}}: \boldsymbol{X} \rightarrow \boldsymbol{Y}$, consists of an increasing function $f$ whose domain is either empty or an initial segment $\left[j_{1}, \alpha_{1}\right]_{\mathbb{N}} \subseteq\left[j_{1}, j_{2}-1\right]_{\mathbb{N}}, j_{1} \leq \alpha_{1}<j_{2}$,

$$
f:\left[j_{1}, \alpha_{1}\right]_{\mathbb{N}} \rightarrow\left[j_{1}, j_{2}-1\right]_{\mathbb{N}},
$$

and, in the later case, of homotopy classes $\left[f_{j}\right]$ of mappings

$$
f_{j}: X_{f(j)} \rightarrow Y_{j}, \quad j=1, \ldots, \alpha_{1},
$$

such that

whenever $j \leq j^{\prime}$.

$$
\left[f_{j}\right]\left[p_{f(j) f\left(j^{\prime}\right)}\right]=\left[q_{j j^{\prime}}\right]\left[f_{j^{\prime}}\right]
$$

A generalization is the notion of an $n$-ladder which will be obtained by fitting together $n 1$-ladders. First, given an $n \in \mathbb{N}$ and any $j_{1}, \ldots, j_{n+1} \in \mathbb{N}$ such that $j_{1}<\cdots<j_{n+1}$, the corresponding ordered $(n+1)$-tuple $\left(j_{1}, \ldots, j_{n+1}\right)$ is denoted by $\boldsymbol{j}^{n}$. The set of all such $(n+1)$-tuples $\boldsymbol{j}^{n}$ is denoted by $\boldsymbol{J}(n)$. 
There is no obstruction to consider the limit case $n \rightarrow \infty$. In this case, an $\omega$-tuple $\boldsymbol{j}^{\omega} \in \boldsymbol{J}(\omega)$ becomes a strictly increasing sequence $\boldsymbol{j}^{\omega}=\left(j_{\lambda}\right)$ in $\mathbb{N}$.

Definition 2.1. Let $\boldsymbol{X}, \boldsymbol{Y} \in \mathrm{Ob} \underline{\mathcal{S}}$, an $n \in \mathbb{N} \cup\{\omega\}$ and a $\boldsymbol{j}^{n} \in \boldsymbol{J}(n)$ be given. An ordered pair $\left(f,\left[f_{j}\right]\right)$ consisting of an increasing (index) function

$$
f: \bigcup_{\lambda=1}^{n}\left[j_{\lambda}, \alpha_{\lambda}\right]_{\mathbb{N}} \rightarrow\left[j_{1}, j_{n+1}-1\right]_{\mathbb{N}}, j_{\lambda} \leq \alpha_{\lambda}<j_{\lambda+1},
$$

and of a set of the homotopy classes of mappings

$$
f_{j}: X_{f(j)} \rightarrow Y_{j}, j \in \bigcup_{\lambda=1}^{n}\left[j_{\lambda}, \alpha_{\lambda}\right]_{\mathbb{N}},
$$

is said to be an n-ladder of $\boldsymbol{X}$ to $\boldsymbol{Y}$ over $\boldsymbol{j}^{n}$, denoted by $\boldsymbol{f}_{\boldsymbol{j}^{n}}: \boldsymbol{X} \rightarrow \boldsymbol{Y}$, provided the following two conditions are satisfied:

$$
\begin{array}{ll}
\left(L(n)_{1}\right) & \left(\forall \lambda \in[1, n]_{\mathbb{N}}\right) f\left(j_{\lambda}\right) \geq j_{\lambda} \wedge f\left(\alpha_{\lambda}\right)<j_{\lambda+1} ; \\
\left(L(n)_{2}\right) & \left(\forall j, j^{\prime} \in \bigcup_{\lambda=1}^{\cup}\left[j_{\lambda}, \alpha_{\lambda}\right]_{\mathbb{N}}\right) j \leq j^{\prime} \Rightarrow\left[f_{j}\right]\left[p_{f(j) f\left(j^{\prime}\right)}\right]=\left[q_{j j^{\prime}}\right]\left[f_{j^{\prime}}\right] . \quad A n
\end{array}
$$

$n$-ladder $\boldsymbol{f}_{j^{n}}$ having an empty $\lambda$-block, i.e. with no mapping for any $j \in\left[j_{\lambda}, j_{\lambda+1}-1\right]_{\mathbb{N}}$, is allowed. In that case, we say that $\alpha_{\lambda}$ is empty. We also admit the empty $n$-ladder of $\boldsymbol{X}$ to $\boldsymbol{Y}$ over $\boldsymbol{j}^{n}$, i.e. the empty set of homotopy classes of mappings for a given $\boldsymbol{j}^{n}$.

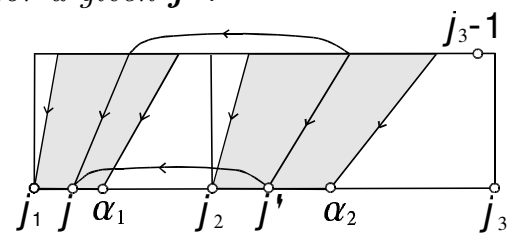

EXAMPLE 2.2. Let $\boldsymbol{f}=\left(f,\left[f_{j}\right]\right): \boldsymbol{X} \rightarrow \boldsymbol{Y}$ be a special morphism of $\underline{\mathcal{S}}$ with $f \geq 1_{\mathbb{N}}$. Given any $n \in \mathbb{N}, \boldsymbol{j}^{n} \in \boldsymbol{J}(n)$ and $\lambda \in[1, n]_{\mathbb{N}}$, put

$$
t_{\lambda}=\max \left\{j \mid f(j)<j_{\lambda+1}, j \in\left[j_{\lambda}, j_{\lambda+1}-1\right]_{\mathbb{N}}\right\} .
$$

For each $\lambda=1, \ldots, n$, if $t_{\lambda}$ does not exist, let $\alpha_{\lambda}$ be empty, and if $t_{\lambda}$ exists, choose any $\alpha_{\lambda} \in\left[j_{\lambda}, t_{\lambda}\right]_{\mathbb{N}}$. Then the restriction of $\boldsymbol{f}$ to $\boldsymbol{X} \mid \boldsymbol{j}^{n}$ and $\boldsymbol{Y} \mid \boldsymbol{j}^{n}$ yields a certain (possible empty) $n$-ladder $\boldsymbol{f}_{\boldsymbol{j}^{n}}=\left(f,\left(\left[f_{j}\right]\right)\right)$ of $\boldsymbol{X}$ to $\boldsymbol{Y}$ over $\boldsymbol{j}^{n}$. One can easily verify conditions $\left(L(n)_{1}\right)$ and $\left(L(n)_{2}\right)$. In the case of $\alpha_{\lambda}=t_{\lambda}$ for every $\lambda$, the ("maximal") $n$-ladder $\boldsymbol{f}_{\boldsymbol{j}^{n}}: \boldsymbol{X} \rightarrow \boldsymbol{Y}$ over $\boldsymbol{j}^{n}$ is said to be induced by $\boldsymbol{f}$. Notice that, for every $\boldsymbol{X}$ and every $\boldsymbol{i}^{n} \in \boldsymbol{J}(n)$, the identity morphism $\mathbf{1}_{\boldsymbol{X}}: \boldsymbol{X} \rightarrow \boldsymbol{X}$ induces the identity $n$-ladder $\mathbf{1}_{\boldsymbol{X} \boldsymbol{i}^{n}}: \boldsymbol{X} \rightarrow \boldsymbol{X}$ over $\boldsymbol{i}^{n}$.

If $\boldsymbol{f}_{\boldsymbol{j}^{n}}: \boldsymbol{X} \rightarrow \boldsymbol{Y}$ and $\boldsymbol{g}_{\boldsymbol{k}^{n}}=\left(g,\left[g_{k}\right]\right): \boldsymbol{Y} \rightarrow \boldsymbol{Z}$ are $n$-ladders, then we compose them only in the case $\boldsymbol{j}^{n}=\boldsymbol{k}^{n}$ by using the ordinary rule, i.e.

$$
\boldsymbol{g}_{\boldsymbol{k}^{n}} \boldsymbol{f}_{\boldsymbol{k}^{n}} \equiv \boldsymbol{u}_{\boldsymbol{k}^{n}}=\left(u,\left[u_{k}\right]\right),
$$

such that $u=f g$ (wherever it is defined) and $u_{k}=g_{k} f_{g(k)}, k \in \bigcup_{\lambda=1}^{n}\left[k_{\lambda}, \gamma_{\lambda}\right]_{\mathbb{N}}$, $\gamma_{\lambda} \leq \beta_{\lambda}$. Clearly, $\boldsymbol{g}_{\boldsymbol{k}^{n}} \boldsymbol{f}_{\boldsymbol{k}^{n}}: \boldsymbol{X} \rightarrow \boldsymbol{Z}$ is an $n$-ladder of $\boldsymbol{X}$ to $\boldsymbol{Z}$ over $\boldsymbol{k}^{n}$. Notice 
that its $\lambda$-block is empty whenever the corresponding block of $\boldsymbol{f}_{\boldsymbol{k}^{n}}$ or $\boldsymbol{g}_{\boldsymbol{k}^{n}}$ is empty, or $g\left(k_{\lambda}\right)>\alpha_{\lambda}$. It is obvious that the composition of $n$-ladders is associative, and that

$$
\begin{aligned}
& \boldsymbol{f}_{\boldsymbol{j}^{n}} \mathbf{1}_{\boldsymbol{X} \boldsymbol{j}^{n}}=\boldsymbol{f}_{\boldsymbol{j}^{n}}, \\
& \mathbf{1}_{\boldsymbol{X} \boldsymbol{i}^{n}} \boldsymbol{g}_{\boldsymbol{i}^{n}}=\boldsymbol{g}_{\boldsymbol{i}^{n}}
\end{aligned}
$$

hold for all $n$-ladders $\boldsymbol{f}_{\boldsymbol{j}^{n}}: \boldsymbol{X} \rightarrow \boldsymbol{Y}$ and $\boldsymbol{g}_{\boldsymbol{i}^{n}}: \boldsymbol{Z} \rightarrow \boldsymbol{X}$. Therefore, for each $\boldsymbol{j}^{n} \in \boldsymbol{J}(n)$, there exists a certain category whose class of objects is Ob $\underline{\mathcal{S}}$, and the sets of morphisms consist of all the corresponding $n$-ladders.

Let $\boldsymbol{f}_{\boldsymbol{j}^{1}}, \boldsymbol{f}_{\boldsymbol{j}^{1}}^{\prime}=\left(f^{\prime},\left[f_{j}^{\prime}\right]\right): \boldsymbol{X} \rightarrow \boldsymbol{Y}$ be 1-ladders over the same $\boldsymbol{j}^{1}$. Then $\boldsymbol{f}_{\boldsymbol{j}^{1}}$ is said to be homotopic to $\boldsymbol{f}_{\boldsymbol{j}^{1}}^{\prime}$ provided they both are empty or there exists a $j_{1}^{*} \in\left[j_{1}, \min \left\{\alpha_{1}, \alpha_{1}^{\prime}\right\}\right]_{\mathbb{N}}$ such that

$$
\begin{aligned}
& \left(\forall j \in\left[j_{1}, j_{1}^{*}\right]_{\mathbb{N}}\right)\left(\exists i=i(j) \in\left[\max \left\{f(j), f^{\prime}(j)\right\}, j_{2}-1\right]_{\mathbb{N}}\right) \\
& {\left[f_{j}\right]\left[p_{f(j) i}\right]=\left[f_{j}^{\prime}\right]\left[p_{f^{\prime}(j) i}\right] .}
\end{aligned}
$$

In the general case of a pair of $n$-ladders the definition of being $m$-homotopic, $m \leq n$, is as follows:

Definition 2.3. Let $n, m \in \mathbb{N} \cup\{\omega\}, m \leq n$, and let $\boldsymbol{f}_{\boldsymbol{j}^{n}}, \boldsymbol{f}_{\boldsymbol{j}^{n}}^{\prime}: \boldsymbol{X} \rightarrow \boldsymbol{Y}$ be n-ladders over the same $\boldsymbol{j}^{n}$. Then $\boldsymbol{f}_{\boldsymbol{j}^{n}}$ is said to be $m$-homotopic to $\boldsymbol{f}_{\boldsymbol{j}^{n}}^{\prime}$, denoted by $\boldsymbol{f}_{\boldsymbol{j}^{n}} \simeq_{m} \boldsymbol{f}_{\boldsymbol{j}^{n}}^{\prime}$, provided, for every $\lambda \in[1, m]_{\mathbb{N}}$, the both $\boldsymbol{f}_{\boldsymbol{j}^{n}}$ and $\boldsymbol{f}_{\boldsymbol{j}^{n}}^{\prime}$ have the $\lambda$-block empty or there exists a $\left.j_{\lambda}^{*} \in\left[j_{\lambda}, \min \left\{\alpha_{\lambda}, \alpha_{\lambda}^{\prime}\right\}\right]_{\mathbb{N}}\right)$ such that

$$
\begin{aligned}
& \left(\forall j \in\left[j_{\lambda}, j_{\lambda}^{*}\right]_{\mathbb{N}}\right)\left(\exists i=i(j) \in\left[\max \left\{f(j), f^{\prime}(j)\right\}, j_{\lambda+1}-1\right]_{\mathbb{N}}\right) \\
& {\left[f_{j}\right]\left[p_{f(j) i}\right]=\left[f_{j}^{\prime}\right]\left[p_{f^{\prime}(j) i}\right] .}
\end{aligned}
$$

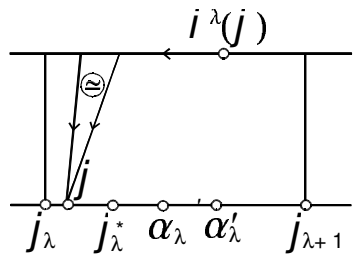

Notice that $\boldsymbol{f}_{\boldsymbol{j}^{n}} \simeq_{m^{\prime}} \boldsymbol{f}_{\boldsymbol{j}^{n}}^{\prime}$ implies $\boldsymbol{f}_{\boldsymbol{j}^{n}} \simeq_{m} \boldsymbol{f}_{\boldsymbol{j}^{n}}^{\prime}$ whenever $m \leq m^{\prime}$. Clearly, the $m$-homotopy relation of $n$-ladders is an equivalence relation on the corresponding set. In the case of $m=n$, we simply write $\boldsymbol{f}_{\boldsymbol{j}^{n}} \simeq \boldsymbol{f}_{\boldsymbol{j}^{n}}^{\prime}$.

A 1-hyperladder (of $\boldsymbol{X}$ to $\boldsymbol{Y}$ ) is a certain family $F_{1}$ of 1-ladders (of $\boldsymbol{X}$ to $\boldsymbol{Y}$ ) indexed by all pairs $\boldsymbol{j}^{1}=\left(j_{1}, j_{2}\right) \in \boldsymbol{J}(1)$. We require that every two elements $j_{1} \leq j_{1}^{\prime}$ of $\mathbb{N}$ admit an $i^{1} \in \mathbb{N}, i^{1} \geq j_{1}^{\prime}$, such that, for every $j_{2}>i^{1}$, the 1-ladder $\boldsymbol{f}_{\boldsymbol{j}^{1}}=\left(f,\left[f_{j}\right]\right) \in F_{1}$, assigned to the pair $\boldsymbol{j}^{1}=\left(j_{1}, j_{2}\right) \in \boldsymbol{J}(1)$, has the following two properties:

the domain $\left[j_{1}, \alpha_{1}\right]_{\mathbb{N}}$ of the index function $f$ contains $\left[j_{1}, j_{1}^{\prime}\right]_{\mathbb{N}}$;

the image $f\left[j_{1}, j_{1}^{\prime}\right]_{\mathbb{N}}$ is contained in $\left[j_{1}, i^{1}\right]_{\mathbb{N}}$. 
Briefly, a family $F_{1}=\left(\boldsymbol{f}_{\boldsymbol{j}^{1}}\right)$ of 1-ladders $\boldsymbol{f}_{\boldsymbol{j}^{1}}: \boldsymbol{X} \rightarrow \boldsymbol{Y}, \boldsymbol{j}^{1} \in \boldsymbol{J}(1)$, s said to be a 1-hyperladder of $\boldsymbol{X}$ to $\boldsymbol{Y}$, provided

$$
\left(\forall j_{1} \in \mathbb{N}\right)\left(\forall j_{1}^{\prime} \geq j_{1}\right)\left(\exists i^{1} \geq j_{1}^{\prime}\right)\left(\forall j_{2}>i^{1}\right)
$$

the index function of the corresponding $\boldsymbol{f}_{\boldsymbol{j}^{1}}=\left(f,\left[f_{j}\right]\right) \in F_{1}$ fulfills the following two conditions:

$$
\alpha_{1} \geq j_{1}^{\prime} \text { and } f\left(j_{1}^{\prime}\right) \leq i^{1} .
$$

Notice that, since $f$ increases, the second condition implies $f(j) \leq i^{1}$ for every $j \in\left[j_{1}, j_{1}^{\prime}\right]_{\mathbb{N}}$.

We are now ready to define the main notion.

Definition 2.4. Let $\boldsymbol{X}, \boldsymbol{Y} \in \mathrm{Ob} \underline{\mathcal{S}}$ and let $n \in \mathbb{N} \cup\{\omega\}$. A family $F_{n}=$ $\left(\boldsymbol{f}_{\boldsymbol{j}^{n}}\right)$ of $n$-ladders $\boldsymbol{f}_{\boldsymbol{j}^{n}}: \boldsymbol{X} \rightarrow \boldsymbol{Y}$, indexed by all $\boldsymbol{j}^{n} \in \boldsymbol{J}(n)$, is said to be an $n$-hyperladder of $\boldsymbol{X}$ to $\boldsymbol{Y}$, denoted by $F_{n}: \boldsymbol{X} \rightarrow \boldsymbol{Y}$, provided

$$
\begin{aligned}
& (\forall m \leq n) \\
& \left(\forall j_{1} \in \mathbb{N}\right)\left(\forall j_{1}^{\prime} \geq j_{1}\right)\left(\exists i^{1} \geq j_{1}^{\prime}\right)\left(\forall j_{2}>i^{1}\right) \ldots \\
& \left(\forall j_{m}>i^{m-1}\right)\left(\forall j_{m}^{\prime} \geq j_{m}\right)\left(\exists i^{m} \geq j_{m}^{\prime}\right)\left(\forall j_{m+1}>i^{m}\right) \\
& \left(\forall j_{m+2}>j_{m+1}\right) \ldots\left(\forall j_{n+1}>j_{n}\right)
\end{aligned}
$$

the index function of the corresponding $\boldsymbol{f}_{\boldsymbol{j}^{n}}=\left(f,\left[f_{j}\right]\right) \in F_{n}$ fulfills the following two conditions:

$$
\begin{aligned}
& \left(S(n, m)_{1}\right)\left(\forall \lambda \in[1, m]_{\mathbb{N}}\right) \alpha_{\lambda} \geq j_{\lambda}^{\prime} ; \\
& \left(S(n, m)_{2}\right)\left(\forall \lambda \in[1, m]_{\mathbb{N}}\right) f\left(j_{\lambda}^{\prime}\right) \leq i^{\lambda} .
\end{aligned}
$$

The set of all n-hyperladders $F_{n}: \boldsymbol{X} \rightarrow \boldsymbol{Y}$ is denoted by $\underline{L}_{n}(\boldsymbol{X}, \boldsymbol{Y})$.

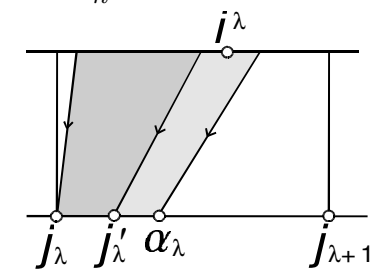

EXAmple 2.5. Let $\boldsymbol{f}=\left(f,\left[f_{j}\right]\right): \boldsymbol{X} \rightarrow \boldsymbol{Y}$ be a special morphism of $\underline{\mathcal{S}}$ with $f \geq 1_{\mathbb{N}}$, and let $n \in \mathbb{N} \cup\{\omega\}$. For every $\boldsymbol{j}^{n} \in \boldsymbol{J}(n)$, let $\boldsymbol{f}_{\boldsymbol{j}^{n}}: \boldsymbol{X} \rightarrow \underline{\boldsymbol{Y}}$ be the $n$-ladder induced by $\boldsymbol{f}$ according to Example 2.2. Then, $\boldsymbol{f}$ yields the family $F_{n}=\left(\boldsymbol{f}_{\boldsymbol{j}^{n}}\right), \boldsymbol{j}^{n} \in \boldsymbol{J}(n)$. Let us show that $F_{n}$ is an $n$-hyperladder of $\boldsymbol{X}$ to $\boldsymbol{Y}$. Given an $m \leq n$, for every $j_{1} \geq 1$ and every $j_{1}^{\prime} \geq j_{1}$ put $i^{1}=f\left(j_{1}^{\prime}\right) ; \ldots$ ; for every $j_{m}>i^{m-1}$ and every $j_{m}^{\prime} \geq j_{m}$ put $i^{m}=f\left(j_{m}^{\prime}\right)$, and let $j_{m+1}>i^{m}$; let $j_{m+2}>j_{m+1}, \ldots, j_{n+1}>j_{n}$. (If $n=\omega$, the construction proceeds by induction.) Then by construction, for every $\lambda \in[1, m]_{\mathbb{N}}, \boldsymbol{f}_{\boldsymbol{j}^{n}}$ has $\alpha_{\lambda} \geq j_{\lambda}^{\prime}$ since $f$ increases and $f\left(j_{\lambda}^{\prime}\right)=i^{\lambda}<j_{\lambda+1}$. Further, $f(j) \leq f\left(j_{\lambda}^{\prime}\right)=i^{\lambda}$ for every $\lambda \in[1, m]_{\mathbb{N}}$ and every $j \in\left[j_{\lambda}, j_{\lambda}^{\prime}\right]_{\mathbb{N}}$. This verifies conditions $\left(S(n, m)_{1,2}\right)$ for $F_{n}$. In such a case, $F_{n}: \boldsymbol{X} \rightarrow \boldsymbol{Y}$ is said to be the $n$-hyperladder induced by a special 
morphism $\boldsymbol{f}: \boldsymbol{X} \rightarrow \boldsymbol{Y}$. In particular, for every $\boldsymbol{X}$, the identity morphism $\mathbf{1}_{\boldsymbol{X}}=\left(1_{\mathbb{N}},\left(\left[1_{X_{i}}\right]\right)\right)$ induces the $n$-hyperladder $1_{\boldsymbol{X} n}=\left(\mathbf{1}_{\boldsymbol{X} \boldsymbol{i}^{n}}\right): \boldsymbol{X} \rightarrow \boldsymbol{X}$, $\boldsymbol{i}^{n} \in \boldsymbol{J}(n)$, (see also Example 2.2).

If $F_{n}=\left(\boldsymbol{f}_{\boldsymbol{j}^{n}}\right): \boldsymbol{X} \rightarrow \boldsymbol{Y}$ and $G_{n}=\left(\boldsymbol{g}_{\boldsymbol{k}^{n}}\right): \boldsymbol{Y} \rightarrow \boldsymbol{Z}, \boldsymbol{k}^{n} \in \boldsymbol{J}(n)$, are $n$-hyperladders, then we compose them by composing the appropriate $n$ ladders $\boldsymbol{f}_{\boldsymbol{j}^{n}}$ and $\boldsymbol{g}_{\boldsymbol{k}^{n}}$ such that $\boldsymbol{j}^{n}=\boldsymbol{k}^{n}$. Hence,

$$
G_{n} F_{n} \equiv U_{n}=\left(\boldsymbol{u}_{\boldsymbol{k}^{n}}\right),
$$

where $\boldsymbol{u}_{\boldsymbol{k}^{n}} \equiv \boldsymbol{g}_{\boldsymbol{k}^{n}} \boldsymbol{f}_{\boldsymbol{k}^{n}}, \boldsymbol{k}^{n} \in \boldsymbol{J}(n)$.

Lemma 2.6. For every $n \in \mathbb{N} \cup\{\omega\}$, the composition of $n$-hyperladders is well defined, i.e.

$$
\left(\forall F_{n} \in \underline{L}_{n}(\boldsymbol{X}, \boldsymbol{Y})\right)\left(\forall G_{n} \in \underline{L}_{n}(\boldsymbol{Y}, \boldsymbol{Z})\right) G_{n} F_{n} \in \underline{L}_{n}(\boldsymbol{X}, \boldsymbol{Z}) .
$$

Proof. Given arbitrary $n \in \mathbb{N} \cup\{\omega\}, m \leq n, F_{n}=\left(\boldsymbol{f}_{\boldsymbol{j}^{n}}\right) \in \underline{L}_{n}(\boldsymbol{X}, \boldsymbol{Y})$ and $G_{n}=\left(\boldsymbol{g}_{\boldsymbol{k}^{n}}\right) \in \underline{L}_{n}(\boldsymbol{Y}, \boldsymbol{Z})$, Definition 2.4 allows the followimg procedure: For every $k_{1} \in \mathbb{N}$ and every $k_{1}^{\prime} \geq k_{1}$ there exists a $j^{1} \geq k_{1}^{\prime}$, and for $j_{1}=k_{1}$ and $j_{1}^{\prime}=j^{1} \geq j_{1}$ there exists an $i^{1} \geq j_{1}^{\prime}$; for every $k_{2}>i^{1} \ldots$; for every $k_{m}>i^{m-1} \geq j^{m-1}$ and every $k_{m}^{\prime} \geq k_{m}$ there exists a $j^{m} \geq k_{m}^{\prime}$, and for $j_{m}=k_{m}$ and $j_{m}^{\prime}=j^{m} \geq j_{m}$ there exists an $i^{m} \geq j_{m}^{\prime}$; let $k_{m+1}=j_{m+1}>i^{m}$, $k_{m+2}=j_{m+2}>k_{m+1}, \ldots, k_{n+1}=j_{n+1}>k_{n}$ (and so on if $n=\omega$ ). Then, $\boldsymbol{j}^{n}=\boldsymbol{k}^{n} \in \boldsymbol{J}(n)$. Observe that, by $\left(S(n, m)_{1,2}\right)$ of $F_{n}, \boldsymbol{f}_{\boldsymbol{j}^{n}} \in F_{n}$ satisfies

$$
\left(\forall \lambda \in[1, m]_{\mathbb{N}}\right) \alpha_{\lambda} \geq j^{\prime \lambda}
$$

and

$$
\left(\forall \lambda \in[1, m]_{\mathbb{N}}\right) f\left(j_{\lambda}^{\prime}\right) \leq i^{\lambda} .
$$

Further, by $\left(S(n, m)_{1,2}\right)$ of $G_{n}, \boldsymbol{g}_{\boldsymbol{k}^{n}} \in G_{n}$ satisfies

$$
\left(\forall \lambda \in[1, m]_{\mathbb{N}}\right) \beta_{\lambda} \geq k_{\lambda}^{\prime}
$$

and

$$
\left(\forall \lambda \in[1, m]_{\mathbb{N}}\right) g\left(k_{\lambda}^{\prime}\right) \leq j^{\lambda} .
$$

Thus, $\boldsymbol{g}_{\boldsymbol{k}^{n}} \boldsymbol{f}_{\boldsymbol{k}^{n}} \in G_{n} F_{n}$ has

$$
\gamma_{\lambda}=\max \left\{k \in\left[k_{\lambda}, \beta_{\lambda}\right]_{\mathbb{N}} \mid g(k) \leq \alpha_{\lambda}\right\},
$$

for every $\lambda \in[1, m]_{\mathbb{N}}$. Moreover, since

$$
\left(\forall \lambda \in[1, m]_{\mathbb{N}}\right) f g\left(k_{\lambda}^{\prime}\right) \leq f\left(j_{\lambda}^{\prime}\right) \leq i^{\lambda},
$$

we infer that

$$
\left(\forall \lambda \in[1, m]_{\mathbb{N}}\right) \gamma_{\lambda} \in\left[k_{\lambda}^{\prime}, \beta_{\lambda}\right]_{\mathbb{N}} .
$$


Therefore, concerning the composition $G_{n} F_{n} \equiv U_{n}=\left(\boldsymbol{u}_{\boldsymbol{k}^{n}}\right), \boldsymbol{u}_{\boldsymbol{k}^{n}} \equiv \boldsymbol{g}_{\boldsymbol{k}^{n}} \boldsymbol{f}_{\boldsymbol{k}^{n}}$, $\boldsymbol{k}^{n} \in \boldsymbol{J}(n)$, we have established that

$$
\begin{aligned}
& (\forall m \leq n) \\
& \left(\forall k_{1} \in \mathbb{N}\right)\left(\forall k_{1}^{\prime} \geq k_{1}\right)\left(\exists i^{1} \geq k_{1}^{\prime}\right)\left(\forall k_{2}>i^{1}\right) \ldots \\
& \left(\forall k_{m}>i^{m-1}\right)\left(\forall k_{m}^{\prime} \geq k_{m}\right)\left(\exists i^{m} \geq k_{m}^{\prime}\right)\left(\forall k_{m+1}>i^{m}\right) \\
& \left(\forall k_{m+2}>k_{m+1}\right) \ldots\left(\forall k_{n+1}>k_{n}\right),
\end{aligned}
$$

the index function $u$ of the corresponding $\boldsymbol{u}_{\boldsymbol{k}^{n}}$ satisfies the following two conditions:

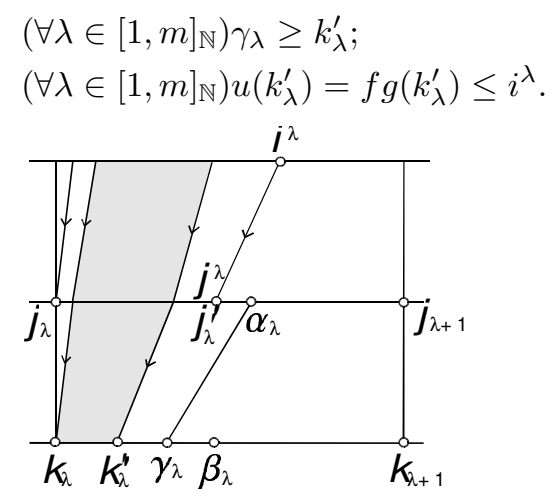

Hence, the family $G_{n} F_{n}=\left(\boldsymbol{g}_{\boldsymbol{k}^{n}} \boldsymbol{f}_{\boldsymbol{k}^{n}}\right)$ fulfills conditions $\left(S(n, m)_{1}\right)$ and $\left(S(n, m)_{2}\right)$. Thus, $G_{n} F_{n} \in \underline{L}_{n}(\boldsymbol{X}, \boldsymbol{Z})$, and the lemma is proved.

Since the composition of $n$-ladders is associative, the composition of $n$ hyperladders is associative. Notice that $1_{\boldsymbol{X} n}=\left(\mathbf{1}_{\boldsymbol{X} \boldsymbol{i}^{n}}\right), \boldsymbol{i}^{n} \in \boldsymbol{J}(n)$, is the identity n-hyperladder on $\boldsymbol{X}$ (see Example 2.5). Indeed,

$$
\begin{aligned}
& F_{n} 1_{\boldsymbol{X} n}=\left(\boldsymbol{f}_{\boldsymbol{j}^{n}}\right)\left(\mathbf{1}_{\boldsymbol{X} \boldsymbol{i}^{n}}\right)=\left(\boldsymbol{f}_{\boldsymbol{j}^{n}} \mathbf{1}_{\boldsymbol{X} \boldsymbol{j}^{n}}\right)=\left(\boldsymbol{f}_{\boldsymbol{j}^{n}}\right)=F_{n}, \\
& 1_{\boldsymbol{X} n} G_{n}=\left(\mathbf{1}_{\boldsymbol{X} \boldsymbol{i}^{n}}\right)\left(\boldsymbol{g}_{\boldsymbol{i}^{n}}\right)=\left(\mathbf{1}_{\boldsymbol{X} \boldsymbol{i}^{n}} \boldsymbol{g}_{\boldsymbol{i}^{n}}\right)=\left(\boldsymbol{g}_{\boldsymbol{i}^{n}}\right)=G_{n}
\end{aligned}
$$

hold for all $n$-hyperladders $F_{n}: \boldsymbol{X} \rightarrow \boldsymbol{Y}$ and $G_{n}: \boldsymbol{Z} \rightarrow \boldsymbol{X}$. Thus, for every $n \in \mathbb{N} \cup\{\omega\}$, there exists a category $\underline{\mathcal{L}}(n)$ consisting of the object class $\operatorname{Ob} \underline{\mathcal{L}}(n)=\operatorname{Ob} \underline{\mathcal{S}}$ and of the class Mor $\underline{\mathcal{L}}(n)$ of all the morphism sets $\underline{L}_{n}(\boldsymbol{X}, \boldsymbol{Y})$.

In order to define a certain equivalence (homotopy) relation on each set $\underline{L}_{n}(\boldsymbol{X}, \boldsymbol{Y})$, let us first consider the simplest case $n=1$. Recall that $\boldsymbol{f}_{\boldsymbol{j}^{1}} \simeq$ $\boldsymbol{f}_{j^{1}}^{\prime}: \boldsymbol{X} \rightarrow \boldsymbol{Y}$ means

$$
\begin{aligned}
& \left(\exists j_{1}^{*} \in\left[j_{1}, \min \left\{\alpha_{1}, \alpha_{1}^{\prime}\right\}\right]_{\mathbb{N}}\right)\left(\forall j \in\left[j_{1}, j_{1}^{*}\right]_{\mathbb{N}}\right)\left(\exists i \in\left[\max \left\{f(j), f^{\prime}(j)\right\}, j_{2}-1\right]_{\mathbb{N}}\right) \\
& {\left[f_{j}\right]\left[p_{f(j) i}\right]=\left[f_{j}^{\prime}\right]\left[p_{f^{\prime}(j) i}\right] .}
\end{aligned}
$$

Let $F_{1}=\left(\boldsymbol{f}_{\boldsymbol{j}^{1}}\right), F_{1}^{\prime}=\left(\boldsymbol{f}_{\boldsymbol{j}^{1}}^{\prime}\right): \boldsymbol{X} \rightarrow \boldsymbol{Y}$ be a pair of 1-hyperladders. Then $F_{1}$ is said to be homotopic. to $F_{1}^{\prime}$, provided every two elements $j_{1} \leq j_{1}^{\prime}$ of $\mathbb{N}$ admit an $i_{*}^{1} \in \mathbb{N}, i_{*}^{1} \geq j_{1}^{\prime}$, such that, for every $j_{2}>i_{*}^{1}$, the corresponding 
1-ladders $\boldsymbol{f}_{\boldsymbol{j}^{1}} \in F_{1}$ and $\boldsymbol{f}_{\boldsymbol{j}^{1}}^{\prime} \in F_{1}^{\prime}$ (assigned to the pair $\boldsymbol{j}^{1}=\left(j_{1}, j_{2}\right) \in \boldsymbol{J}(1)$ ) are homotopic, $\boldsymbol{f}_{\boldsymbol{j}^{1}} \simeq \boldsymbol{f}_{\boldsymbol{j}^{1}}^{\prime}$ and, in addition, the occuring $j_{1}^{*} \geq j_{1}^{\prime}$ and $i=$ $i\left(j_{1}^{\prime}\right) \leq i_{*}^{1}$.

Briefly, $F_{1} \simeq F_{1}^{\prime}$ provided

$$
\left(\forall j_{1} \in \mathbb{N}\right)\left(\forall j_{1}^{\prime} \geq j_{1}\right)\left(\exists i_{*}^{1} \geq j_{1}^{\prime}\right)\left(\forall j_{2}>i_{*}^{1}\right)
$$

the corresponding $\boldsymbol{f}_{\boldsymbol{j}^{1}} \in F_{1}$ and $\boldsymbol{f}_{\boldsymbol{j}^{1}}^{\prime} \in F_{1}^{\prime}$ are homotopic, $\boldsymbol{f}_{\boldsymbol{j}^{1}} \simeq \boldsymbol{f}_{\boldsymbol{j}^{1}}^{\prime}$, such that $j_{1}^{*} \geq j_{\lambda}^{\prime}$ and $i=i\left(j_{1}^{\prime}\right) \leq i_{*}^{1}$.

Notice that the last condition implies that $i=i(j) \leq i_{*}^{1}$ for every $j \in$ $\left[j_{1}, j_{1}^{\prime}\right]_{\mathbb{N}}$.

Definition 2.7. Let $\boldsymbol{X}, \boldsymbol{Y} \in \mathrm{Ob} \underline{\mathcal{S}}, n \in \mathbb{N} \cup\{\omega\}$ and let $F_{n}=\left(\boldsymbol{f}_{\boldsymbol{j}^{n}}\right), F_{n}^{\prime}=$ $\left(\boldsymbol{f}_{\boldsymbol{j}^{n}}^{\prime}\right): \boldsymbol{X} \rightarrow \boldsymbol{Y}$ be n-hyperladders. Then $F_{n}$ is said to be homotopic to $F_{n}^{\prime}$, denoted by $F_{n} \simeq F_{n}^{\prime}$, provided

$$
\begin{aligned}
& (\forall m \leq n) \\
& \left(\forall j_{1} \in \mathbb{N}\right)\left(\forall j_{1}^{\prime} \geq j_{1}\right)\left(\exists i_{*}^{1} \geq j_{1}^{\prime}\right)\left(\forall j_{2}>i_{*}^{1}\right) \ldots \\
& \left(\forall j_{m}>i_{*}^{m-1}\right)\left(\forall j_{m}^{\prime} \geq j_{m}\right)\left(\exists i_{*}^{m} \geq j_{m}^{\prime}\right)\left(\forall j_{m+1}>i_{*}^{m}\right) \\
& \left(\forall j_{m+2}>j_{m+1}\right) \ldots\left(\forall j_{n+1}>j_{n}\right)
\end{aligned}
$$

the corresponding n-ladders $\boldsymbol{f}_{\boldsymbol{j}^{n}} \in F_{n}$ and $\boldsymbol{f}_{\boldsymbol{j}^{n}}^{\prime} \in F_{n}^{\prime}$ satisfy the following condition:

$$
(H(n, m)) \boldsymbol{f}_{\boldsymbol{j}^{n}} \simeq_{m} \boldsymbol{f}_{\boldsymbol{j}^{n}},
$$

i.e. for every $\lambda \in[1, m]_{\mathbb{N}}$ there exists a $\left.j_{\lambda}^{*} \in\left[j_{\lambda}, \min \left\{\alpha_{\lambda}, \alpha_{\lambda}^{\prime}\right\}\right]_{\mathbb{N}}\right)$ for which

$$
\begin{aligned}
& \left(\forall j \in\left[j_{\lambda}, j_{\lambda}^{*}\right]_{\mathbb{N}}\right)\left(\exists i=i(j) \in\left[\max \left\{f(j), f^{\prime}(j)\right\}, j_{\lambda+1}-1\right]_{\mathbb{N}}\right) \\
& {\left[f_{j}\right]\left[p_{f(j) i}\right]=\left[f_{j}^{\prime}\right]\left[p_{f^{\prime}(j) i}\right],}
\end{aligned}
$$

such that, in addition,

$$
\left(\forall \lambda \in[1, m]_{\mathbb{N}}\right), j_{\lambda}^{*} \geq j_{\lambda}^{\prime}
$$

and

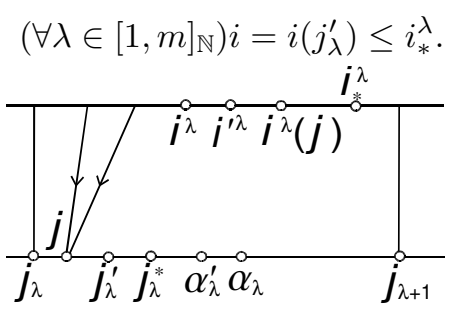

Observe that the last condition implies that $i=i(j) \leq i_{*}^{\lambda}$, for every $\lambda$ and every $j \in\left[j_{\lambda}, j_{\lambda}^{\prime}\right]_{\mathbb{N}}$. Further, for the indices $i_{*}^{\lambda}$ in Definition 2.7 and for the indices $i^{\lambda}, i^{\prime \lambda}$ (for $F_{n}, F_{n}^{\prime}$ respectively) in Definition 2.4,

$$
\left(\forall \lambda \in[1, m]_{\mathbb{N}}\right) i_{*}^{\lambda} \geq \max \left\{i^{\lambda}, i^{\prime \lambda}\right\}
$$

must hold. 
Lemma 2.8. For every $n \in \mathbb{N} \cup\{\omega\}$, the homotopy relation of the $n$ hyperladders is a natural equivalence relation in the category $\underline{\mathcal{L}}(n)$.

Proof. Since the $m$-homotopy of $n$-ladders is an equivalence relation, to prove that the homotopy of $n$-hyperladders is an equivalence relation, we only need to show that it is transitive. The verification is straightforward by means of $(H(n, m))$, of $F_{n} \simeq F_{n}^{\prime}$ and $F_{n}^{\prime} \simeq F_{n}^{\prime \prime}, m=1, \ldots, n$. Namely, given an $m \leq n$, one should choose $i_{*}^{\lambda}=\max \left\{i_{*}^{\prime \lambda}, i_{*}^{\prime \prime \lambda}\right\}$ and $j_{\lambda}^{*}=\min \left\{j_{\lambda}^{\prime *}, j_{\lambda}^{\prime \prime *}\right\}$ for every $\lambda \in[1, m]_{\mathbb{N}}$.

Let $F_{n}, F_{n}^{\prime} \in \underline{L}_{n}(\boldsymbol{X}, \boldsymbol{Y})$ such that $F_{n} \simeq F_{n}^{\prime}$, and let $G_{n}=\left(\boldsymbol{g}_{\boldsymbol{k}^{n}}\right) \in \underline{L}_{n}(\boldsymbol{Y}, \boldsymbol{Z})$. Then, for each $m \leq n$, Definitions 2.4 and 2.7 allow the following procedure: $F$ or every $k_{1}$ and every $k_{1}^{\prime} \geq k_{1}$ there exists a $j^{1} \geq k_{1}^{\prime}$, and for $j_{1}=k_{1}$ and $j_{1}^{\prime}=j^{1} \geq j_{1}$ there exists an $i_{*}^{1} \geq j_{1}^{\prime} ; \ldots$; for every $k_{m}>i_{*}^{m-1}$ and every $k_{m}^{\prime} \geq k_{m}$ there exists a $j^{m} \geq k_{m}^{\prime}$, and for $j_{m}=k_{m}$ and $j_{m}^{\prime}=j^{m} \geq j_{m}$ there exists an $i_{*}^{m} \geq j_{m}^{\prime}$; let $k_{m+1}=j_{m+1}>i_{*}^{m}, k_{m+2}=j_{m+2}>k_{m+1}, \ldots$, $k_{n+1}=j_{n+1}>k_{n}$ (and so on if $n=\omega$ ). Then, $\boldsymbol{j}^{n}=\boldsymbol{k}^{n} \in \boldsymbol{J}(n)$. Consider the $n$-ladders $\boldsymbol{f}_{k^{n}} \in F_{n}, \boldsymbol{f}_{\boldsymbol{k}^{n}}^{\prime} \in F_{n}^{\prime}$ and $\boldsymbol{g}_{\boldsymbol{k}^{n}} \in G_{n}$. By condition $\left(S(n, m)_{2}\right)$ of $G_{n}$,

$$
\left(\forall \lambda \in[1, m]_{\mathbb{N}}\right) g\left(k_{\lambda}^{\prime}\right) \leq j^{\lambda}=j_{\lambda}^{\prime},
$$

and by condition $(H(n, m))$ of $F_{n} \simeq F_{n}^{\prime}$,

$$
\left(\forall \lambda \in[1, m]_{\mathbb{N}}\right)\left(\forall j \in\left[j_{\lambda}, j_{\lambda}^{*}\right]_{\mathbb{N}}\right)\left[f_{j}\right]\left[p_{f(j) i^{\lambda}(j)}\right]=\left[f_{j}^{\prime}\right]\left[p_{f^{\prime}(j) i^{\lambda}(j)}\right],
$$

where $j_{\lambda}^{*} \geq j_{\lambda}^{\prime}$ and $i^{\lambda}(j) \leq i_{*}^{\lambda}, j \in\left[j_{\lambda}, j_{\lambda}^{\prime}\right]$. Thus,

$$
\begin{aligned}
& \left(\forall \lambda \in[1, m]_{\mathbb{N}}\right)\left(\exists k_{\lambda}^{*} \geq k_{\lambda}^{\prime}\right)\left(\forall k \in\left[k_{\lambda}, k_{\lambda}^{*}\right]_{\mathbb{N}}\right) \\
& {\left[g_{k}\right]\left[f_{g(k)}\right]\left[p_{f(g(k)) i^{\lambda}(g(k))}\right]=\left[g_{k}\right]\left[f_{g(k)}^{\prime}\right]\left[p_{f^{\prime}(g(k)) i^{\lambda}(g(k))}\right] .}
\end{aligned}
$$

Therefore, concerning the compositions $G_{n} F_{n}$ and $G_{n} F_{n}^{\prime}$, for each $m \leq n$ the following is fulfilled:

$$
\begin{aligned}
& \left(\forall k_{1} \in \mathbb{N}\right)\left(\forall k_{1}^{\prime} \geq k_{1}\right)\left(\exists i_{*}^{1} \geq k_{1}^{\prime}\right)\left(\forall k_{2}>i_{*}^{1}\right) \ldots \\
& \left(\forall k_{m}>i_{*}^{m-1}\right)\left(\forall k_{m}^{\prime} \geq k_{m}\right)\left(\exists i_{*}^{m} \geq k_{m}^{\prime}\right)\left(\forall k_{m+1}>i_{*}^{m}\right) \\
& \left(\forall k_{m+2}>k_{m+1}\right) \ldots\left(\forall k_{n+1}>k_{n}\right),
\end{aligned}
$$

the following condition is satisfied:

$$
\boldsymbol{g}_{\boldsymbol{k}^{n}} \boldsymbol{f}_{\boldsymbol{k}^{n}} \simeq_{m} \boldsymbol{g}_{\boldsymbol{k}^{n}} \boldsymbol{f}_{\boldsymbol{k}^{n}}^{\prime}
$$

such that

$$
\left(\forall \lambda \in[1, m]_{\mathbb{N}}\right), k_{\lambda}^{*} \geq k_{\lambda}^{\prime}
$$

and

$$
\left(\forall \lambda \in[1, m]_{\mathbb{N}}\right) i\left(k_{\lambda}^{\prime}\right) \equiv i^{\lambda}\left(g\left(k_{\lambda}^{\prime}\right)\right) \leq i_{*}^{\lambda} .
$$




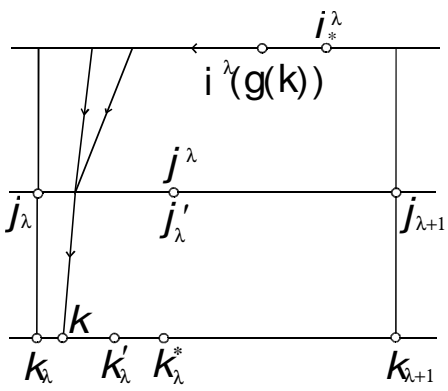

This fulfills condition $(H(n, m))$ for $G_{n} F_{n}$ and $G_{n} F_{n}^{\prime}$. Consequently, $G_{n} F_{n} \simeq$ $G_{n} F_{n}^{\prime}$.

Let $H_{n}=\left(\boldsymbol{h}_{\boldsymbol{i}^{n}}\right) \in \underline{L}_{n}(\boldsymbol{W}, \boldsymbol{X})$ and let $F_{n}, F_{n}^{\prime} \in \underline{L}_{n}(\boldsymbol{X}, \boldsymbol{Y})$ such that $F_{n} \simeq F_{n}^{\prime}$. Then, for every $m \leq n$, Definitions 2.7 and 2.4 allow the following procedure: $F$ or every $j_{1}$ and every $j_{1}^{\prime} \geq j_{1}$ there exists an $i_{*}^{1} \geq j_{1}^{\prime}$, and for $i_{1}=j_{1}$ and $i_{1}^{\prime}=i_{*}^{1} \geq i_{1}$ there exists a $t^{1} \geq i_{1}^{\prime} ; \ldots$; for every $j_{m}>t^{m-1}$ and every $j_{m}^{\prime} \geq j_{m}$ there exists an $i_{*}^{m} \geq j_{m}^{\prime}$, and for $i_{m}=j_{m}$ and $i_{m}^{\prime}=i_{*}^{m} \geq i_{m}$ there exists a $t^{m} \geq i_{m}^{\prime}$; let $j_{m+1}=i_{m+1}>t^{m}, j_{m+2}=i_{m+2}>j_{m+1}, \ldots$, $j_{n+1}=i_{n+1}>j_{n}$ (and so on if $n=\omega$ ). Then, $\boldsymbol{i}^{n}=\boldsymbol{j}^{n} \in \boldsymbol{J}(n)$. Consider the $n$-ladders $\boldsymbol{h}_{\boldsymbol{j}^{n}} \in H_{n}, \boldsymbol{f}_{\boldsymbol{j}^{n}} \in F_{n}$ and $\boldsymbol{f}_{\boldsymbol{j}^{n}}^{\prime} \in F_{n}^{\prime}$. By condition $(H(n, m))$ of $F_{n} \simeq F_{n}^{\prime}$,

$$
\left(\forall \lambda \in[1, m]_{\mathbb{N}}\right)\left(\forall j \in\left[j_{\lambda}, j_{\lambda}^{*}\right]_{\mathbb{N}}\right)\left[f_{j}\right]\left[p_{f(j) i^{\lambda}(j)}\right]=\left[f_{j}^{\prime}\right]\left[p_{f^{\prime}(j) i^{\lambda}(j)}\right],
$$

where $j_{\lambda}^{*} \geq j_{\lambda}^{\prime}$ and $i^{\lambda}(j) \leq i_{*}^{\lambda}$. By $\left(S(n, m)_{2}\right)$ of $H_{n}$,

$$
\left(\forall \lambda \in[1, m]_{\mathbb{N}}\right) h\left(i_{\lambda}^{\prime}\right) \leq t^{\lambda} .
$$

Thus,

$$
\begin{aligned}
& \left(\forall \lambda \in[1, m]_{\mathbb{N}}\right)\left(\exists j_{\lambda}^{\prime *} \geq j_{\lambda}^{*}\right)\left(\forall j \in\left[j_{\lambda}, j_{\lambda}^{\prime *}\right]_{\mathbb{N}}\right) \\
& {\left[f_{j}\right]\left[h_{f(j)}\right]\left[u_{h(f(j)) t^{\lambda}}\right]=\left[f_{j}^{\prime}\right]\left[h_{f^{\prime}(j)}\right]\left[u_{h\left(f^{\prime}(j)\right) t^{\lambda}}\right] .}
\end{aligned}
$$

Therefore, concerning the compositions $F_{n} H_{n}$ and $F_{n}^{\prime} H_{n}$, for each $m \leq n$ the following is fulfilled:

$$
\begin{aligned}
& \left(\forall j_{1} \in \mathbb{N}\right)\left(\forall j_{1}^{\prime} \geq j_{1}\right)\left(\exists t_{*}^{1}=t^{1}\right)\left(\forall j_{2}>t_{*}^{1}\right) \ldots \\
& \left(\forall j_{m}>t_{*}^{m-1}\right)\left(\forall j_{m}^{\prime} \geq j_{m}\right)\left(\exists t_{*}^{m}=t^{m} \geq j_{m}^{\prime}\right)\left(\forall j_{m+1}>t_{*}^{m}\right) \\
& \left(\forall j_{m+2}>j_{m+1}\right) \ldots\left(\forall j_{n+1}>j_{n}\right),
\end{aligned}
$$

the relation $\boldsymbol{f}_{\boldsymbol{j}^{n}} \boldsymbol{h}_{\boldsymbol{j}^{n}} \simeq_{m} \boldsymbol{f}_{\boldsymbol{j}^{n}}^{\prime} \boldsymbol{h}_{\boldsymbol{j}^{n}}$ is satisfied and

$$
\left(\forall \lambda \in[1, m]_{\mathbb{N}}\right), j_{\lambda}^{* *} \geq k_{\lambda}^{\prime}
$$

and

$$
\left(\forall \lambda \in[1, m]_{\mathbb{N}}\right) t^{\lambda}\left(j_{\lambda}^{*}\right) \equiv h\left(i^{\lambda}\left(j_{\lambda}^{\prime *}\right)\right) \leq t_{*}^{\lambda} .
$$




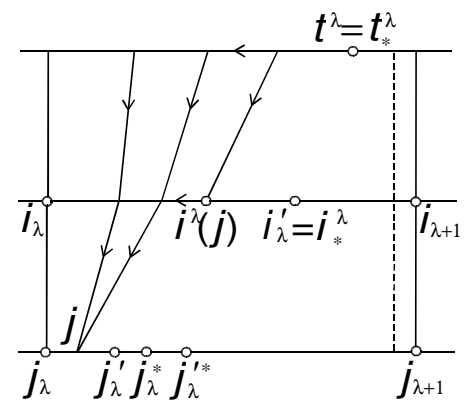

This fulfills condition $(H(n, m))$ for $F_{n} H_{n}$ and $F_{n}^{\prime} H_{n}$, and thus, $F_{n} H_{n} \simeq$ $F_{n}^{\prime} H_{n}$.

The homotopy class $\left[F_{n}\right]$ of an $F_{n} \in \underline{L}_{n}(\boldsymbol{X}, \boldsymbol{Y})$, denoted by $\boldsymbol{F}_{n}: \boldsymbol{X} \rightarrow \boldsymbol{Y}$, is said to be an $\underline{S}_{n}$-morphism. The obtained results are summarized in the next theorem:

TheOREM 2.9. For every $n \in \mathbb{N} \cup\{\omega\}$, there exists a quotient (homotopy) category $\underline{\mathcal{S}}(n)=\underline{\mathcal{L}}(n) / \simeq$ consisting of the class of objects $\mathrm{Ob}(\underline{\mathcal{S}}(n))=\mathrm{Ob} \underline{\mathcal{S}}$ and of the class $\operatorname{Mor}(\underline{\mathcal{S}}(n))$ of all the sets of $\underline{S}_{n}$-morphisms,

$$
\underline{\mathcal{S}}(n)(\boldsymbol{X}, \boldsymbol{Y})=\left\{\boldsymbol{F}_{n}=\left[F_{n}\right] \mid F_{n} \in \underline{L}_{n}(\boldsymbol{X}, \boldsymbol{Y})\right\}=\underline{\mathcal{L}}(n)(\boldsymbol{X}, \boldsymbol{Y}) / \simeq,
$$

with composition defined by

$$
\boldsymbol{G}_{n} \boldsymbol{F}_{n}=\left[G_{n}\right]\left[F_{n}\right]=\left[G_{n} F_{n}\right],
$$

and with the identity $\underline{S}_{n}$-morphism $\mathbf{1}_{\boldsymbol{X} n}=\left[1_{\boldsymbol{X}_{n}}\right]$ on each object $\boldsymbol{X} \in$ $\mathrm{Ob}(\underline{\mathcal{S}}(n))$.

Consider now a sequence $\boldsymbol{F} \equiv\left(\boldsymbol{F}_{n}\right)$ of $\underline{S}_{n}$-morphisms $\boldsymbol{F}_{n} \in \underline{\mathcal{S}}(n)(\boldsymbol{X}, \boldsymbol{Y})$, $n \in \mathbb{N}$, as a new arrow of $\boldsymbol{X}$ to $\boldsymbol{Y}$. Such a sequence $\boldsymbol{F}: \boldsymbol{X} \rightarrow \boldsymbol{Y}$ is said to be an $\underline{S}_{\mathbb{N}}$-morphism of $\boldsymbol{X}$ to $\boldsymbol{Y}$. If any $\underline{S}_{\mathbb{N}}$-morphism $\boldsymbol{G}=\left(\boldsymbol{G}_{n}\right): \boldsymbol{Y} \rightarrow \boldsymbol{Z}$ is given, we define the composition of $\boldsymbol{F}$ and $\boldsymbol{G}$ coordinatewise, i.e.

$$
\boldsymbol{G} \boldsymbol{F}=\left(\boldsymbol{G}_{n}\right)\left(\boldsymbol{F}_{n}\right)=\left(\boldsymbol{G}_{n} \boldsymbol{F}_{n}\right) .
$$

Clearly, $\boldsymbol{G F}: \boldsymbol{X} \rightarrow \boldsymbol{Z}$ is a well-defined $\underline{S}_{\mathbb{N}}$-morphism. Moreover, this composition is associative, because of

$$
\begin{aligned}
\boldsymbol{H}(\boldsymbol{G} \boldsymbol{F}) & =\left(\boldsymbol{H}_{n}\right)\left(\left(\boldsymbol{G}_{n} \boldsymbol{F}_{n}\right)\right)=\left(\boldsymbol{H}_{n}\left(\boldsymbol{G}_{n} \boldsymbol{F}_{n}\right)\right) \\
& =\left(\left(\boldsymbol{H}_{n} \boldsymbol{G}_{n}\right) \boldsymbol{F}_{n}\right)=\left(\left(\boldsymbol{H}_{n} \boldsymbol{G}_{n}\right)\right)\left(\boldsymbol{F}_{n}\right)=(\boldsymbol{H} \boldsymbol{G}) \boldsymbol{F} .
\end{aligned}
$$

Further, there exists the identity $\underline{S}_{\mathbb{N}}$-morphism $\mathbf{1}_{\boldsymbol{X}}=\left(\mathbf{1}_{\boldsymbol{X} n}\right): \boldsymbol{X} \rightarrow \boldsymbol{X}$ on each object $\boldsymbol{X}$. Indeed,

$$
(\forall \boldsymbol{F}: \boldsymbol{X} \rightarrow \boldsymbol{Y}) \boldsymbol{F} \mathbf{1}_{\boldsymbol{X}}=\left(\boldsymbol{F}_{n} \mathbf{1}_{\boldsymbol{X} n}\right)=\left(\boldsymbol{F}_{n}\right)=\boldsymbol{F}
$$

and

$$
(\forall \boldsymbol{G}: \boldsymbol{Z} \rightarrow \boldsymbol{X}) \mathbf{1}_{\boldsymbol{X}} \boldsymbol{G}=\left(\mathbf{1}_{\boldsymbol{X} n} \boldsymbol{G}_{n}\right)=\left(\boldsymbol{G}_{n}\right)=\boldsymbol{G} .
$$

Hence, the following theorem is obtained: 
THEOREM 2.10. There exists a (homotopy) category $\underline{\mathcal{S}}(\mathbb{N})$ consisting of the class of objects $\operatorname{Ob}(\underline{\mathcal{S}}(\mathbb{N}))=\operatorname{Ob} \underline{\mathcal{S}}$ and of the class $\operatorname{Mor}(\underline{\mathcal{S}}(\mathbb{N}))$ of all the sets of $\underline{S}_{\mathbb{N}}$-morphisms,

$$
\underline{\mathcal{S}}(\mathbb{N})(\boldsymbol{X}, \boldsymbol{Y})=\left\{\boldsymbol{F} \mid \boldsymbol{F}=\left(\boldsymbol{F}_{n}\right), \boldsymbol{F}_{n}=\left[F_{n}\right] \in \underline{\mathcal{S}}(n)(\boldsymbol{X}, \boldsymbol{Y}), n \in \mathbb{N}\right\},
$$

with composition defined by

$$
\boldsymbol{G F}=\left(\boldsymbol{G}_{n}\right)\left(\boldsymbol{F}_{n}\right)=\left(\boldsymbol{G}_{n} \boldsymbol{F}_{n}\right),
$$

and with the identity $\underline{S}_{\mathbb{N}}$-morphism $\mathbf{1}_{\boldsymbol{X}}=\left(\mathbf{1}_{\boldsymbol{X}_{n}}\right)=\left(\left[1_{\boldsymbol{X}_{n}}\right]\right)$ on each object $\boldsymbol{X} \in \operatorname{Ob}(\underline{\mathcal{S}}(\mathbb{N}))$.

Let us now exhibit some functorial relationships between the previously constructed categories.

Theorem 2.11. For every pair $n, n^{\prime} \in \mathbb{N} \cup\{\omega\}, n \leq n^{\prime}$, there exists a restriction functor $\underline{R}_{n n^{\prime}}: \underline{\mathcal{S}}\left(n^{\prime}\right) \rightarrow \underline{\mathcal{S}}(n)$ (which is not unique) keeping the objects fixed. $\underline{R}_{n n}$ is the identity functor. Furthermore, for all $n \leq n^{\prime} \leq n^{\prime \prime}$, there exist $\underline{R}_{n n^{\prime}}, \underline{R}_{n^{\prime} n^{\prime \prime}}$ and $\underline{R}_{n n^{\prime \prime}}$ such that $\underline{R}_{n n^{\prime}} \underline{R}_{n^{\prime} n^{\prime \prime}}=\underline{R}_{n n^{\prime \prime}}$, i.e. the diagram

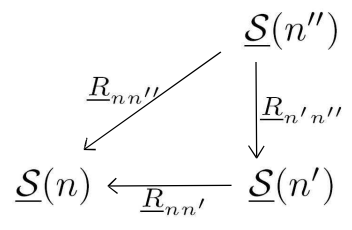

commutes.

Proof. Let $n \leq n^{\prime}$ and let $F_{n^{\prime}}=\left(\boldsymbol{f}_{\boldsymbol{j}^{n^{\prime}}}\right) \in \underline{L}_{n^{\prime}}(\boldsymbol{X}, \boldsymbol{Y})$. For every $\boldsymbol{j}^{n^{\prime}} \in$ $\boldsymbol{J}\left(n^{\prime}\right)$, let $\boldsymbol{f}_{\boldsymbol{j}^{n^{\prime}}}^{n^{\prime}}$ be the restriction of $\boldsymbol{f}_{\boldsymbol{j}^{n^{\prime}}} \in F_{n^{\prime}}$ to $\boldsymbol{j}^{n} \in \boldsymbol{J}(n)$. This yields, for all $\boldsymbol{j}^{n}$, a certain family $\left(\boldsymbol{f}_{\boldsymbol{j}^{n}}^{\alpha}\right)$ of $n$-ladders $\boldsymbol{f}_{\boldsymbol{j}^{n}}^{\alpha}: \boldsymbol{X} \rightarrow \boldsymbol{Y}, \alpha \equiv \boldsymbol{j}^{n^{\prime}} \in A\left(\boldsymbol{j}^{n}\right) \subseteq$ $\boldsymbol{J}\left(n^{\prime}\right)$. Let

$$
\psi: \boldsymbol{J}(n) \rightarrow \boldsymbol{J}\left(n^{\prime}\right), \psi\left(\boldsymbol{j}^{n}\right)=\boldsymbol{j}^{n^{\prime}},
$$

be an injective function such that

$$
\left(\forall \lambda \in[1, n]_{\mathbb{N}}\right) j_{\lambda}^{\prime}=j_{\lambda} \text {, and } j_{n+1}^{\prime}=j_{n+1} .
$$

Notice that, for every $\boldsymbol{j}^{n} \in \boldsymbol{J}(n)$, the value $\psi\left(\boldsymbol{j}^{n}\right) \in A\left(\boldsymbol{j}^{n}\right)$. Let $F_{n}=\left(\boldsymbol{f}_{\boldsymbol{j}^{n}}^{\psi\left(\boldsymbol{j}^{n}\right)}\right)$ be indexed by all $\boldsymbol{j}^{n} \in \boldsymbol{J}(n)$. One readily sees that conditions $\left(S\left(n^{\prime}, m\right)_{1,2}\right)$, $m \leq n^{\prime}$, of $F_{n^{\prime}}$ imply conditions $\left(S(n, m)_{1,2}\right), m \leq n$, for $F_{n}$. Thus, $F_{n} \in$ $\underline{L}_{n}(\boldsymbol{X}, \boldsymbol{Y})$. Hence, the correspondence $F_{n^{\prime}} \mapsto F_{n}$ yields, for every pair $\boldsymbol{X}, \boldsymbol{Y}$, function

$$
\Psi \equiv \Psi_{\psi, \boldsymbol{X}, \boldsymbol{Y}}: \underline{L}_{n^{\prime}}(\boldsymbol{X}, \boldsymbol{Y}) \rightarrow \underline{L}_{n}(\boldsymbol{X}, \boldsymbol{Y}), \Psi\left(F_{n^{\prime}}\right)=F_{n} .
$$

Notice that this construction assures that $\Psi\left(1_{\boldsymbol{X}_{n^{\prime}}}\right)=1_{\boldsymbol{X} n}$. Moreover, since the composition of $n$-hyperladders is defined coordinatewise (by indices), the following fact is obvious: 
If $G_{n^{\prime}} F_{n^{\prime}}=U_{n^{\prime}} \mapsto U_{n}, G_{n^{\prime}} \mapsto G_{n}$ and $F_{n^{\prime}} \mapsto F_{n}$, then $U_{n}=G_{n} F_{n}$.

It implies that $\Psi\left(G_{n^{\prime}} F_{n^{\prime}}\right)=\Psi\left(G_{n^{\prime}}\right) \Psi\left(F_{n^{\prime}}\right)$. Therefore, the function $\psi$ induces a functor $\Psi: \underline{\mathcal{L}}\left(n^{\prime}\right) \rightarrow \underline{\mathcal{L}}(n)$ keeping the objects fixed. Let $F_{n^{\prime}}^{\prime}=$ $\left(\boldsymbol{f}_{\boldsymbol{j}^{n^{\prime}}}^{\prime}\right) \in \underline{L}_{n^{\prime}}(\boldsymbol{X}, \boldsymbol{Y})$ be an $n^{\prime}$-hyperladder such that $F_{n^{\prime}}^{\prime} \simeq F_{n^{\prime}}$, and let $F_{n}^{\prime}=$ $\left(\boldsymbol{f}_{\boldsymbol{j}^{n}}^{\prime}\right) \in \underline{L}_{n}(\boldsymbol{X}, \boldsymbol{Y})$ be obtained in the same way by means of $F_{n^{\prime}}^{\prime}$ and (the same function) $\psi$. Then one readily sees that conditions $\left(H\left(n^{\prime}, m\right)\right), m \leq n^{\prime}$, of $F_{n^{\prime}} \simeq F_{n^{\prime}}^{\prime}$ imply conditions $(H(n, m)), m \leq n$, for $F_{n}=\Psi\left(F_{n^{\prime}}\right)$ and $F_{n}^{\prime}=\Psi\left(F_{n^{\prime}}^{\prime}\right)$, i.e. $\Psi\left(F_{n^{\prime}}\right) \simeq \Psi\left(F_{n^{\prime}}^{\prime}\right)$ Thus, the functor $\Psi: \underline{\mathcal{L}}\left(n^{\prime}\right) \rightarrow \underline{\mathcal{L}}(n)$ induces the functor $\underline{R}_{n n^{\prime}}^{\psi}: \underline{\mathcal{S}}\left(n^{\prime}\right) \rightarrow \underline{\mathcal{S}}(n)$, which keeps the objects fixed and $\underline{R}_{n n^{\prime}}^{\psi}\left(\boldsymbol{F}_{n^{\prime}}\right)=\underline{R}_{n n^{\prime}}^{\psi}\left(\left[F_{n^{\prime}}\right]\right)=\left[\Psi\left(F_{n^{\prime}}\right)\right] \equiv \boldsymbol{F}_{n}^{\psi}$.

Notice that $n^{\prime}=n$ implies that $\psi=1_{\underline{J}(n)}$ is unique. Hence, $\Psi=1_{\underline{\mathcal{L}}(n)}$ is unique, and thus, the functor $\underline{R}_{n n}^{1}=1_{\underline{\mathcal{S}}(n)}$ is the unique identity functor. Finally, if $n \leq n^{\prime} \leq n^{\prime \prime}$ then, for every pair $\psi, \psi^{\prime}$ as above, the functors $\underline{R}_{n n^{\prime}}^{\psi}: \underline{\mathcal{S}}\left(n^{\prime}\right) \rightarrow \underline{\mathcal{S}}(n), \underline{R}_{n^{\prime} n^{\prime \prime}}^{\psi^{\prime}}: \underline{\mathcal{S}}\left(n^{\prime \prime}\right) \rightarrow \underline{\mathcal{S}}\left(n^{\prime}\right)$ and $\underline{R}_{n n^{\prime \prime}}^{\psi^{\prime} \psi}: \underline{\mathcal{S}}\left(n^{\prime \prime}\right) \rightarrow \underline{\mathcal{S}}(n)$ satisfy $\underline{R}_{n n^{\prime}}^{\psi} \underline{R}_{n^{\prime} n^{\prime \prime}}^{\psi^{\prime}}=\underline{R}_{n n^{\prime \prime}}^{\psi^{\prime} \psi}$.

Let $\psi=\left(\psi_{n}\right)$ be a sequence of injective functions

$$
\psi_{n}: \boldsymbol{J}(n) \rightarrow \boldsymbol{J}(n+1), \psi_{n}\left(\boldsymbol{j}^{n}\right)=\boldsymbol{j}^{n^{\prime}}, n \in \mathbb{N},
$$

such that

$$
(\forall n \in \mathbb{N})\left(\forall \lambda \in[1, n]_{\mathbb{N}}\right) j_{\lambda}^{\prime}=j_{\lambda} \text { and } j_{n+1}^{\prime}=j_{n+1} .
$$

Then, according to Theorem 2.11, $\psi$ determines a subcategory $\underline{\mathcal{S}}^{\psi}(\mathbb{N}) \subseteq \underline{\mathcal{S}}(\mathbb{N})$ containing all the objects, while an $\boldsymbol{F} \in \underline{\mathcal{S}}^{\psi}(\mathbb{N})(\boldsymbol{X}, \boldsymbol{Y})$ is a sequence $\left(\boldsymbol{F}_{n}\right)$ satisfying $\boldsymbol{F}_{n}=\underline{R}_{n, n+1}^{\psi_{n}}\left(\boldsymbol{F}_{n+1}\right)$ for every $n \in \mathbb{N}$.

Theorem 2.12. For every $n \in \mathbb{N}$, there exists an $n$-projection functor $\underline{P}_{n}: \underline{\mathcal{S}}(\mathbb{N}) \rightarrow \underline{\mathcal{S}}(n)$, which keeps the objects fixed. Further, for every sequence $\psi=\left(\psi_{n}\right)$ as above and every pair $n \leq n^{\prime}$, the following diagram of the functors commutes:

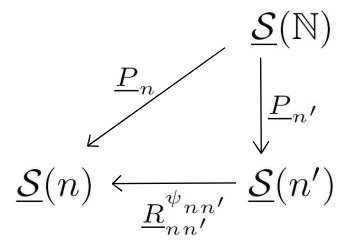

$\left(\psi_{n n^{\prime}}\right.$ denotes the composition $\psi_{n} \psi_{n+1} \cdots \psi_{n^{\prime}}$.)

Proof. For every $n$ and every $\boldsymbol{X} \in \mathrm{Ob}(\underline{\mathcal{S}})$, put $\underline{P}_{n}(\boldsymbol{X})=\boldsymbol{X}$ and, for every $\boldsymbol{F}=\left(\boldsymbol{F}_{n}\right) \in \underline{\mathcal{S}}(\mathbb{N})(\boldsymbol{X}, \boldsymbol{Y})$, put $\underline{P}_{n}(\boldsymbol{F})=\boldsymbol{F}_{n} \in \underline{\mathcal{S}}(n)(\boldsymbol{X}, \boldsymbol{Y})$. Then $\underline{P}_{n}\left(\mathbf{1}_{\boldsymbol{X}}\right)=\mathbf{1}_{\boldsymbol{X} n}$ and

$$
\underline{P}_{n}(\boldsymbol{G F})=\underline{P}_{n}\left(\left(\boldsymbol{G}_{n} \boldsymbol{F}_{n}\right)\right)=\boldsymbol{G}_{n} \boldsymbol{F}_{n}=\underline{P}_{n}(\boldsymbol{G}) \underline{P}_{n}(\boldsymbol{F}) .
$$

Thus, $\underline{P}_{n}: \underline{\mathcal{S}}(\mathbb{N}) \rightarrow \underline{\mathcal{S}}(n)$ is a functor. The rest of the proof is straightforward by Theorem 2.11 and by the definition of $\underline{\mathcal{S}}^{\psi}(\mathbb{N})$. 
THEOREM 2.13. For every sequence $\psi=\left(\psi_{n}\right)$ as above, the sequence $\left(\underline{P}_{n}\right): \underline{\mathcal{S}}^{\psi}(\mathbb{N}) \rightarrow\left(\underline{\mathcal{S}}(n), \underline{R}_{n n^{\prime}}^{\psi_{n n^{\prime}}}, \mathbb{N}\right)$ of the projection functors $\underline{P}_{n}: \underline{\mathcal{S}}^{\psi}(\mathbb{N}) \rightarrow$ $\underline{\mathcal{S}}(n)$ is the (inverse) limit of the inverse sequence $\left(\underline{\mathcal{S}}(n), \underline{R}_{n n^{\prime}}^{\psi_{n n^{\prime}}}, \mathbb{N}\right)$ in the category CAT of all small categories.

Proof. Recall that every compact metrizable space admits an embedding into the Hilbert cube. Therefore, we may consider $H c \mathcal{M}$ to be a small category. Consequently, $\underline{\mathcal{S}}$ as well as each $\underline{\mathcal{S}}(n), n \in \mathbb{N}$, and $\underline{\mathcal{S}}(\mathbb{N})$ may be considered to be small categories. Thus, according to Theorems 2.11 and 2.12 , we only need to verify the universal property. Let $\left(A_{n}\right)$ be a sequence of functors $A_{n}: \mathcal{A} \rightarrow \underline{\mathcal{S}}(n), n \in \mathbb{N}$, in CAT such that, for a given sequence $\psi=\left(\psi_{n}\right)$,

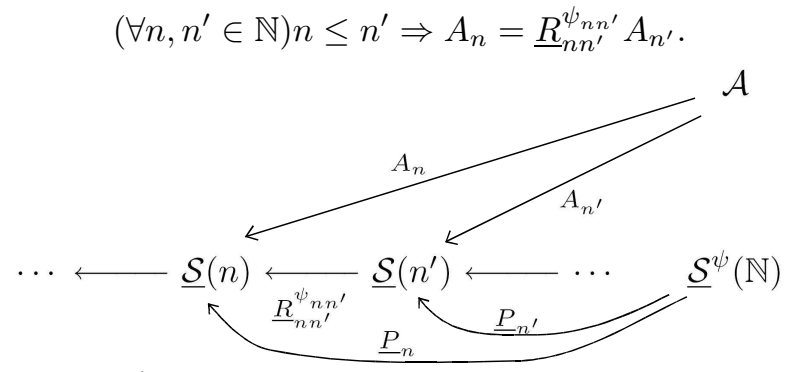

Since the functors $\underline{R}_{n n^{\prime}}^{\psi_{n n^{\prime}}}$ are identities on the object class, it follows that

$$
(\forall \xi \in \mathrm{Ob} \mathcal{A})\left(\forall n, n^{\prime} \in \mathbb{N}\right) A_{n}(\xi)=A_{n^{\prime}}(\xi) .
$$

Let us denote $A_{n}(\xi)=\boldsymbol{X}_{\xi}$ and, for a $u \in \mathcal{A}(\xi, \theta), A_{n}(u)=\boldsymbol{F}_{n}^{u} \in$ $\underline{\mathcal{S}}(n)\left(\boldsymbol{X}_{\xi}, \boldsymbol{Y}_{\theta}\right)$. For every $\xi \in \operatorname{Ob} \mathcal{A}$, put $A(\xi)=\boldsymbol{X}_{\xi}$, and for every $u \in \mathcal{A}(\xi, \theta)$, put $A(u)=\left(A_{n}(u)\right)=\left(\boldsymbol{F}_{n}^{u}\right) \equiv \boldsymbol{F}^{u} \in \underline{\mathcal{S}}^{\psi}(\mathbb{N})\left(\boldsymbol{X}_{\xi}, \boldsymbol{Y}_{\theta}\right)$. Notice that $A(u)$ is well defined because of $A_{n}=\underline{R}_{n n^{\prime}}^{\psi_{n n^{\prime}}} A_{n^{\prime}}, n \leq n^{\prime}$. Since every $A_{n}$ is a functor,

$$
A\left(1_{\xi}\right)=\left(A_{n}\left(1_{\xi}\right)\right)=\left(\mathbf{1}_{\boldsymbol{X}_{\xi} n}\right)=\mathbf{1}_{\boldsymbol{X}_{\xi}} \in \underline{\mathcal{S}}^{\psi}(\mathbb{N})\left(\boldsymbol{X}_{\xi}, \boldsymbol{X}_{\xi}\right)
$$

and

$$
\begin{aligned}
A(v u) & =\left(A_{n}(v u)\right)=\left(A_{n}(v) A_{n}(u)\right)=\left(\boldsymbol{G}_{n}^{v} \boldsymbol{F}_{n}^{u}\right)= \\
& =\left(\boldsymbol{G}_{n}^{v}\right)\left(\boldsymbol{F}_{n}^{u}\right)=\left(A_{n}(v)\right)\left(A_{n}(u)\right)=A(v) A(u) .
\end{aligned}
$$

Thus, $A: \mathcal{A} \rightarrow \underline{\mathcal{S}}^{\psi}(\mathbb{N})$ is a functor. Clearly, $\underline{P}_{n} A=A_{n}$ holds for every $n \in \mathbb{N}$. Let $B: \mathcal{A} \rightarrow \underline{\mathcal{S}}^{\psi}(\mathbb{N})$ be any functor satisfying $\underline{P}_{n} B=A_{n}$ for every $n \in \mathbb{N}$. Then

$$
(\forall \xi \in \mathrm{Ob} \mathcal{A})(\forall n \in \mathbb{N}) B(\xi)=\underline{P}^{n}(B(\xi))=A_{n}(\xi)=A(\xi) .
$$

Further, if $u \in \mathcal{A}(\xi, \theta)$ and $B(u) \equiv \boldsymbol{F}^{\prime u}=\left(\boldsymbol{F}_{n}^{\prime u}\right) \in \underline{\mathcal{S}}^{\psi}(\mathbb{N})\left(\boldsymbol{X}_{\xi}, \boldsymbol{Y}_{\theta}\right)$, then

$$
\boldsymbol{F}_{n}^{\prime u}=\underline{P}_{n}\left(\boldsymbol{F}^{\prime u}\right)=\underline{P}_{n}(B(u))=\underline{P}_{n} B(u) \in \underline{\mathcal{S}}(n)\left(\boldsymbol{X}_{\xi}, \boldsymbol{Y}_{\theta}\right), n \in \mathbb{N} .
$$

Therefore,

$$
(\forall u \in \mathcal{A}(\xi, \theta)) B(u)=\left(\underline{P}_{n} B(u)\right)=\left(A_{n}(u)\right)=A(u) .
$$


Thus, $B=A$, and the universal property is verified.

\section{The Shape CATEgory versus the subshape CATEgories}

We consider hereby the relationships between the "realizing" shape category $\underline{\mathcal{S}} h=\underline{\mathcal{S}} / \simeq$ of compacta and the previously constructed subshape categories $\underline{\mathcal{S}}(n), n \in \mathbb{N} \cup\{\omega\}$, as well as $\underline{\mathcal{S}}(\mathbb{N})$ and $\underline{\mathcal{S}}^{\psi}(\mathbb{N})$. First of all, we point out the following two simple facts:

Lemma 3.1. (i) Let $\boldsymbol{f}, \boldsymbol{f}^{\prime}: \boldsymbol{X} \rightarrow \boldsymbol{Y}$ be special morphisms of $\underline{\mathcal{S}}$ with $f, f^{\prime} \geq 1_{\mathbb{N}}$, and letn $\in \mathbb{N} \cup\{\omega\}$. If $\boldsymbol{f} \simeq \boldsymbol{f}^{\prime}$ then the induced $n$ hyperladders $F_{n}, F_{n}^{\prime}: \boldsymbol{X} \rightarrow \boldsymbol{Y}$ are homotopic, i.e. $F_{n} \simeq F_{n}^{\prime}$.

(ii) Let $\boldsymbol{f}: \boldsymbol{X} \rightarrow \boldsymbol{Y}$ and $\boldsymbol{g}: \boldsymbol{Y} \rightarrow \boldsymbol{Z}$ be special morphisms of $\underline{\mathcal{S}}$ with $f, g \geq 1_{\mathbb{N}}$, and let $n \in \mathbb{N} \cup\{\omega\}$. If $F_{n} \in \underline{L}_{n}(\boldsymbol{X}, \boldsymbol{Y}), G_{n} \in \underline{L}_{n}(\boldsymbol{Y}, \boldsymbol{Z})$ and $H_{n} \in \underline{L}_{n}(\boldsymbol{X}, \boldsymbol{Z})$ are the n-hyperladders induced by $\boldsymbol{f}, \boldsymbol{g}$ and $\boldsymbol{g} \boldsymbol{f}$ respectively, then $G_{n} F_{n}=H_{n}$.

Proof. Recall that the homotopy relation $f \simeq f^{\prime}$ in $\underline{\mathcal{S}}$ means

$$
(\forall j \in \mathbb{N})\left(\exists i \geq f(j), f^{\prime}(j)\right)\left[f_{j}\right]\left[p_{f(j) i}\right]=\left[f_{j}^{\prime}\right]\left[p_{f^{\prime}(j) i}\right] .
$$

Therefore (see Examples 2.2 and 2.5), for every $n \in \mathbb{N} \cup\{\omega\}$, one can easily choose the appropriate indices and obtain a desired $\boldsymbol{j}^{n}$ which confirms that $F_{n} \simeq F_{n}^{\prime}$. This proves claim (i). Further, if $\boldsymbol{f}$ and $\boldsymbol{g}$ are special morphisms with $f, g \geq 1_{\mathbb{N}}$, then the induced $n$-hyperladders $F_{n}, G_{n}$ and $H_{n}$ obviously satisfy $\boldsymbol{h}_{\boldsymbol{k}^{n}}=\boldsymbol{g}_{\boldsymbol{k}^{n}} \boldsymbol{f}_{\boldsymbol{k}^{n}}$ for every $\boldsymbol{k}^{n} \in \boldsymbol{J}(n)$. Thus, claim (ii) follows.

Theorem 3.2. (i) For every $n \in \mathbb{N} \cup\{\omega\}$, there exists a functor $\underline{T}_{n}$ : $\underline{\mathcal{S}} h \rightarrow \underline{\mathcal{S}}(n)$ keeping the objects fixed. Further, for every restriction functor $\underline{R}_{n n^{\prime}}: \underline{\mathcal{S}}\left(n^{\prime}\right) \rightarrow \underline{\mathcal{S}}(n)$ the following diagram commutes:

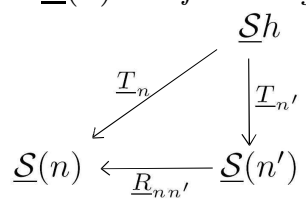

(ii) There exists a unique functor $\underline{T}: \underline{\mathcal{S}} h \rightarrow \underline{\mathcal{S}}(\mathbb{N})$, which keeps the objects fixed and

$$
(\forall n \in \mathbb{N}) \underline{P}_{n} \underline{T}=\underline{T}_{n},
$$

where $\underline{P}_{n}: \underline{\mathcal{S}}(\mathbb{N}) \rightarrow \underline{\mathcal{S}}(n)$ is the $n$-projection functor.

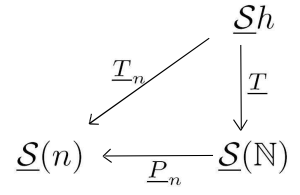

Proof. For every $n$ and every $\boldsymbol{X} \in \mathrm{Ob} \underline{\mathcal{S}} h$, put $\underline{T}_{n}(\boldsymbol{X})=\boldsymbol{X}$. For every $\Phi \equiv[\boldsymbol{f}] \in \underline{\mathcal{S}} h(\boldsymbol{X}, \boldsymbol{Y}), \boldsymbol{f}=\left(f,\left[f_{j}\right]\right): \boldsymbol{X} \rightarrow \boldsymbol{Y}$, put $\underline{T}_{n}(\Phi) \equiv \boldsymbol{F}_{n}=\left[F_{n}\right] \in$ 
$\underline{\mathcal{S}}(n)(\boldsymbol{X}, \boldsymbol{Y})$, where $F_{n}=\left(\boldsymbol{f}_{\boldsymbol{j}^{n}}\right) \in \underline{L}_{n}(\boldsymbol{X}, \boldsymbol{Y}), \boldsymbol{j}^{n} \in \boldsymbol{J}(n)$, is the $n$-hyperladder induced by a special representative $\boldsymbol{f} \in \Phi$ (see Examples 2.2 and 2.5). By Lemma 3.1 (i), the correspondence $\Phi \mapsto \underline{T}_{n}(\Phi)=\boldsymbol{F}_{n}$ is well defined. Namely, $\boldsymbol{f} \simeq \boldsymbol{f}^{\prime}$ implies $F_{n} \simeq F_{n}^{\prime}$. Further, $\underline{T}_{n}\left(1_{\boldsymbol{X}}\right)=\left[1_{\boldsymbol{X}_{n}}\right]=\mathbf{1}_{\boldsymbol{X} n}$, and, by Lemma 3.1 (ii),

$$
\begin{aligned}
\underline{T}_{n}(\Psi \Phi) & =\underline{T}_{n}([\boldsymbol{g} \boldsymbol{f}])=\left[G_{n} F_{n}\right]=\left[G_{n}\right]\left[F_{n}\right] \\
& =\underline{T}_{n}([\boldsymbol{g}]) \underline{T}_{n}([\boldsymbol{f}])=\underline{T}_{n}(\Psi) \underline{T}_{n}(\Phi) .
\end{aligned}
$$

Hence, $\underline{T}_{n}: \underline{\mathcal{S}} h \rightarrow \underline{\mathcal{S}}(n)$ is a functor. Further, notice (see the proof of Theorem 2.11) that a restriction functor $\underline{R}_{n n^{\prime}}^{\psi}: \underline{\mathcal{S}}\left(n^{\prime}\right) \rightarrow \underline{\mathcal{S}}(n)$ restricted to the image $\underline{T}_{n^{\prime}}[\underline{\mathcal{S}} h] \subseteq \underline{\mathcal{S}}\left(n^{\prime}\right)$ does not depend on $\psi$ any more. (The restrictions of all the $n^{\prime}$-ladders $\boldsymbol{f}_{\boldsymbol{j}^{n^{\prime}}}$ to a $\boldsymbol{j}^{n}$ are the same $n$-ladder $\boldsymbol{f}_{\boldsymbol{j}^{n}}$.) Thus, $\underline{R}_{n n^{\prime}} \underline{T}_{n^{\prime}}=\underline{T}_{n}$. This proves claim (i).

To prove claim (ii), put $\underline{T}=\left(\underline{T}_{n}\right)$, i.e. $\underline{T}(\boldsymbol{X})=\boldsymbol{X}$ and

$$
\underline{T}(\Phi)=\left(\underline{T}_{n}(\Phi)\right)=\left(\boldsymbol{F}_{n}\right)=\boldsymbol{F} \in \underline{\mathcal{S}}(\mathbb{N})(\boldsymbol{X}, \boldsymbol{Y}) .
$$

Hence, the image $\underline{T}[\underline{\mathcal{S}} h] \subseteq \underline{\mathcal{S}}(\mathbb{N})$ is an $\underline{\mathcal{S}}^{\psi}(\mathbb{N})$-type subcategory. Therefore, the conclusion follows by Theorem 2.13.

Corollary 3.3. For every $n \in \mathbb{N} \cup\{\omega\}$ there exists an $n$-subshape functor $\underline{S}_{n}: H c \mathcal{M} \rightarrow \underline{\mathcal{S}}(n)$, which keeps the objects fixed and $\underline{T}_{n} \underline{S}=\underline{S}_{n}$, where $\underline{S}: H c \mathcal{M} \rightarrow \underline{\mathcal{S}} h$ is the (ordinary) shape functor. Further, there exists an $\underline{\mathbb{N}}$-subshape functor $\underline{\Sigma}: H c \mathcal{M} \rightarrow \underline{\mathcal{S}}(\mathbb{N})$, which keeps the objects fixed and $\underline{T S}=\underline{\Sigma}$.

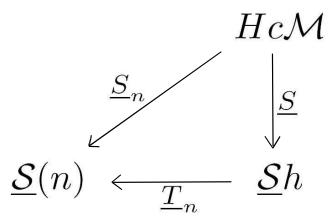

Proof. Put $\underline{S}_{n}(X)=\boldsymbol{X}$, where $\boldsymbol{X}$ is a compact ANR-sequence associated with $X(\lim \boldsymbol{X}=X)$. Further, if $f: X \rightarrow Y$ is an ordinary mapping, put $\underline{S}_{n}([f])=\boldsymbol{F}_{n}=\left[F_{n}\right]$, where $F_{n}=\left(\boldsymbol{f}_{\boldsymbol{j}^{n}}\right) \in \underline{L}_{n}(\boldsymbol{X}, \boldsymbol{Y})$ is the $n$-hyperladder induced by a special morphism $\boldsymbol{f}: \boldsymbol{X} \rightarrow \boldsymbol{Y}$, and $\boldsymbol{f}$ is associated with the mapping $f$. The functor $\underline{\Sigma}$ is defined by means of the sequence $\left(\underline{S}_{n}\right)$. The conclusions follow by Theorem 3.2.

TheOREm 3.4. (i) Two inverse sequences $\boldsymbol{X}, \boldsymbol{Y}$ of compact ANR's are isomorphic in the shape category $\underline{S}$ h (i.e. they have the same shape) if and only if they are isomorphic objects of the category $\underline{\mathcal{S}}(\omega)$.

(ii) There exists a subcategory of $\underline{\mathcal{S}}(\omega)$ which is isomorphic to the shape category $\underline{\mathcal{S}} h$.

Proof. The necessity part of (i) holds by Theorem 3.2 (i). Conversely, let $\boldsymbol{X} \cong \boldsymbol{Y}$ in $\underline{\mathcal{S}}(\omega)$. Then there exists a pair of $\omega$-hyperladders $F_{\omega}=\left(\boldsymbol{f}_{\boldsymbol{j}^{\omega}}\right) \in$ 
$\underline{L}_{\omega}(\boldsymbol{X}, \boldsymbol{Y}), \boldsymbol{j}_{\omega}^{\omega} \in \boldsymbol{J}(\omega), G_{\omega}=\left(\boldsymbol{g}_{i^{\omega}}\right) \in \underline{L}_{\omega}(\boldsymbol{Y}, \boldsymbol{X}), \boldsymbol{i}^{\omega} \in \boldsymbol{J}(\omega)$, such that $G_{\omega} F_{\omega} \simeq 1_{\boldsymbol{X} \omega}$ and $F_{\omega} G_{\omega} \simeq 1_{\boldsymbol{Y} \omega}$. Notice that, by Definitions 2.4 and 2.7 ( $m=n=\omega)$, one has the following inductive construction:

For $j_{1}=1$ and every $j_{1}^{\prime} \geq j_{1}$, there exist an $i^{1} \geq j_{1}^{\prime}$ and a $j_{*}^{1} \geq j_{1}^{\prime}$; for $i_{1}=j_{1}$ and $i_{1}^{\prime}=i^{1}$, there exist a $j^{1} \geq i_{1}^{\prime}$ and an $i_{*}^{1} \geq i_{1}^{\prime}$; for $j_{1}$ and $j_{1}^{\prime \prime}=\max \left\{j_{*}^{1}, j^{1}\right\}$, there exists an $i^{\prime 1} \geq j_{1}^{\prime \prime}$; put $i^{\prime \prime 1}=\max \left\{i_{*}^{1}, i^{\prime 1}\right\} ; \ldots$;

for every $j_{\lambda+1}>i^{\prime \prime \lambda}$ and every $j_{\lambda+1}^{\prime} \geq j_{\lambda+1}$, there exist an $i^{\lambda+1} \geq j_{\lambda+1}^{\prime}$ and a $j_{*}^{\lambda+1} \geq j_{\lambda+1}^{\prime}$; for $i_{\lambda+1}=j_{\lambda+1}$ and $i_{\lambda+1}^{\prime}=i^{\lambda+1}$, there exist a $j^{\lambda+1} \geq i_{\lambda+1}^{\prime}$ and an $i_{*}^{\lambda+1} \geq i_{\lambda+1}^{\prime}$; for $j_{\lambda+1}$ and $j_{\lambda+1}^{\prime \prime}=\max \left\{j_{*}^{\lambda+1}, j^{\prime \lambda+1}\right\}$, there exists an $i^{\prime \lambda+1} \geq j_{\lambda+1}^{\prime \prime}$, put $i^{\prime \prime \lambda+1}=\max \left\{i_{*}^{\lambda+1}, i^{\prime \lambda+1}\right\}$; for every $j_{\lambda+2}>i^{\prime \prime \lambda+1} \ldots$, and so on, by induction on $\lambda \in \mathbb{N}$.

Then $\boldsymbol{j}^{\omega}=\boldsymbol{i}^{\omega} \in \boldsymbol{J}(\omega)$ and

$$
\boldsymbol{g}_{\boldsymbol{i}^{\omega}} \boldsymbol{f}_{\boldsymbol{i}^{\omega}} \simeq \mathbf{1}_{\boldsymbol{X} \boldsymbol{i}^{\omega}}
$$

such that

$$
(\forall \lambda \in \mathbb{N}), i_{\lambda}^{*} \geq i_{\lambda}^{\prime}
$$

and

as well as

$$
(\forall \lambda \in \mathbb{N}) i\left(i_{\lambda}^{\prime}\right) \leq i_{*}^{\lambda},
$$

$\boldsymbol{f}_{\boldsymbol{j}^{\omega}} \boldsymbol{g}_{\boldsymbol{j}^{\omega}} \simeq \mathbf{1}_{\boldsymbol{Y} \boldsymbol{j}^{\omega}}$

such that

$$
(\forall \lambda \in \mathbb{N}), j_{\lambda}^{*} \geq j_{\lambda}^{\prime}
$$

and

$$
(\forall \lambda \in \mathbb{N}) j\left(j_{\lambda}^{\prime}\right) \leq j_{*}^{\lambda} .
$$

Observe that the $\boldsymbol{\omega}$-ladder $\boldsymbol{f}_{\boldsymbol{j}^{\omega}}$ yields the morphism of inverse sequences $\boldsymbol{f}$ : $\boldsymbol{X} \rightarrow \boldsymbol{Y}$. (Each "missing" mapping $f_{j}: X_{f(j)} \rightarrow Y_{j}$ is the composition $q_{j j^{\prime}} f_{j^{\prime}}$, where $j^{\prime} \geq j$ is the closest index such that there exists an $f_{j^{\prime}}$ belonging to $\boldsymbol{f}_{\boldsymbol{j}^{\omega}}$.) In the same way, the $\omega$-ladder $\boldsymbol{g}_{\boldsymbol{i}^{\omega}}$ yields the morphism of inverse sequences $\boldsymbol{g}: \boldsymbol{Y} \rightarrow \boldsymbol{X}$. Then the compositions $\boldsymbol{g} \boldsymbol{f}: \boldsymbol{X} \rightarrow \boldsymbol{X}$ and $\boldsymbol{f g}: \boldsymbol{Y} \rightarrow \boldsymbol{Y}$ satisfy the following condition:

$$
(\forall i \in \mathbb{N})\left(\exists i^{\prime} \geq \max \{i, f g(i)\}\right)\left[g_{i}\right]\left[f_{g(i)}\right]\left[p_{f g(i) i^{\prime}}\right]=\left[p_{i i^{\prime}}\right]
$$

and

$$
(\forall j \in \mathbb{N})\left(\exists j^{\prime} \geq \max \{j, g f(j)\}\right)\left[f_{j}\right]\left[g_{f(j)}\right]\left[q_{g f(j) j^{\prime}}\right]=\left[q_{j j^{\prime}}\right] .
$$

This means that $\boldsymbol{g} \boldsymbol{f} \simeq \mathbf{1}_{\boldsymbol{X}}$ and $\boldsymbol{f g} \simeq \mathbf{1}_{\boldsymbol{Y}}$, and thus $\boldsymbol{X} \cong \boldsymbol{Y}$ in the category $\underline{\mathcal{S}} h$.

To prove claim (ii), one should only observe that all the $\omega$-hyperladders $F_{\omega}=\left(\boldsymbol{f}_{\boldsymbol{j}^{\omega}}\right)$ which are induced by the special morphisms (see Example 2.5 and Lemma 3.1) form a subcategory of $\underline{\mathcal{S}}(\omega)$. More precisely, the restriction of the functor $\underline{T}_{\omega}: \underline{\mathcal{S}} h \rightarrow \underline{\mathcal{S}}(\omega)$ to its image $\underline{T}_{\omega}[\underline{\mathcal{S}} h] \subseteq \underline{\mathcal{S}}(\omega)$ is an isomorphism of categories. 
Recall that the shape category $\mathcal{S} h$ of compacta, $\mathrm{Ob}(\mathcal{S} h)=\mathrm{Ob}(c \mathcal{M})$, is realized via the (shape) category $\underline{\mathcal{S}} h$ of compact $A N R$-inverse sequences. This means that

$$
\mathcal{S} h(X, Y) \approx \underline{\mathcal{S}} h(\boldsymbol{X}, \boldsymbol{Y}),
$$

where $\boldsymbol{X}, \boldsymbol{Y}$ are any with $X, Y$ associated compact $A N R$-sequences respectively. In the same way, we introduce, beside the realizing subshape categories $\underline{\mathcal{S}}(n), \underline{\mathcal{S}}(\mathbb{N})$ and $\underline{\mathcal{S}}^{\psi}(\mathbb{N})$, the corresponding subshape categories $\mathcal{S}(n), \mathcal{S}(\mathbb{N})$ and $\mathcal{S}^{\psi}(\mathbb{N})$ on compacta, respectively. For instance, the corresponding category of $\underline{\mathcal{S}}(n)$ is the category $\mathcal{S}(n), n \in \mathbb{N} \cup\{\omega\}$, determined by

$$
\mathrm{Ob}(\mathcal{S}(n))=\mathrm{Ob}(c \mathcal{M})
$$

and

$$
\mathcal{S}(n)(X, Y) \approx \underline{\mathcal{S}}(n)(\boldsymbol{X}, \boldsymbol{Y})
$$

where $\boldsymbol{X}, \boldsymbol{Y}$ are any with $X, Y$ associated compact $A N R$-sequences, respectively. The definition is correct since, by Theorem 3.2, if $\boldsymbol{X}^{\prime} \cong \boldsymbol{X}$ and $\boldsymbol{Y}^{\prime} \cong \boldsymbol{Y}$ in $\underline{\mathcal{S}} h$, then $\underline{\mathcal{S}}(n)\left(\boldsymbol{X}^{\prime}, \boldsymbol{Y}^{\prime}\right) \approx \underline{\mathcal{S}}(n)(\boldsymbol{X}, \boldsymbol{Y})$. Clearly, every fact of the previous section, as well as of the first part of this section, yields a corresponding fact in this setting. The functors corresponding to $\underline{R}_{n n^{\prime}}, \underline{P}_{n}, \underline{T}_{n}, \underline{T}$ and $\underline{\Sigma}$ are denoted by $R_{n n^{\prime}}, P_{n}, T_{n}, T$ and $\Sigma$, respectively.

Corollary 3.5. (i) For every $n \in \mathbb{N} \cup\{\omega\}$, there exists a functor $T_{n}: \mathcal{S} h \rightarrow \mathcal{S}(n)$, which keeps the objects fixed and is such that

$$
R_{n n^{\prime}} T_{n^{\prime}}=T_{n}, n \leq n^{\prime},
$$

where $R_{n n^{\prime}}: \mathcal{S}\left(n^{\prime}\right) \rightarrow \mathcal{S}(n)$ is any restriction functor.

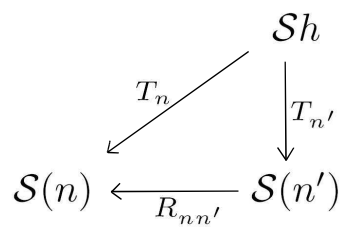

(ii) There exists a unique functor $T: \mathcal{S} h \rightarrow \mathcal{S}(\mathbb{N})$, which keeps the objects fixed and is such that

$$
(\forall n \in \mathbb{N}) P_{n} T=T_{n},
$$

where $P_{n}: \mathcal{S} \rightarrow \mathcal{S}(n)$ is the n-projection functor.

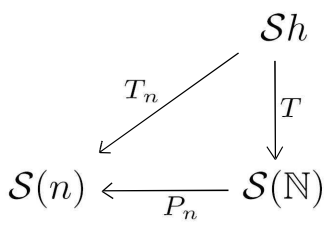


(iii) For every $n \in \mathbb{N} \cup\{\omega\}$, there exists an $n$-subshape functor $S_{n}: H c \mathcal{M} \rightarrow$ $\mathcal{S}(n)$, which keeps the objects fixed and is such that $T_{n} S=S_{n}$, where $S: H c \mathcal{M} \rightarrow \mathcal{S h}$ is the (ordinary) shape functor.

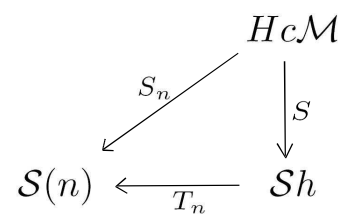

(iv) There exists an $\mathbb{N}$-subshape functor $\Sigma: H c \mathcal{M} \rightarrow \mathcal{S}(\mathbb{N})$, which keeps the objects fixed and is such that $T S=\Sigma$.

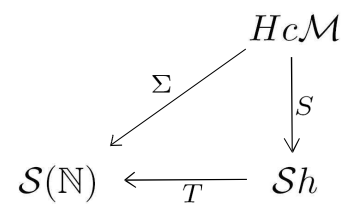

(v) Two compacta $X$ and $Y$ have the same shape if and only if they are isomorphic objects of the category $\mathcal{S}(\omega)$. Furthermore, the restriction of the functor $T_{\omega}: \mathcal{S} h \rightarrow \mathcal{S}(\omega)$ to its image $T_{\omega}[\mathcal{S} h] \subseteq \mathcal{S}(\omega)$ is an isomorphism of categories.

Proof. Statements (i) and (ii) correspond to Theorem 3.2, statements (iii) and (iv) correspond to Corollary 3.3, while statement (v) corresponds to Theorem 3.4.

REMARK 3.6. In Definition 2.7 one may reduce condition $(H(n, m))$ to $m \in[1, s]_{\mathbb{N}}$ for a fixed $s \leq n$. This yields the ("relative") $s$-homotopy relation on appropriate sets of $n$-hyperladders, $F_{n} \simeq_{s} F_{n}^{\prime}$. Then $F_{n} \simeq F_{n}^{\prime}$ is the special ("absolute") case of $F_{n} \simeq_{s} F_{n}^{\prime}$ for $s=n$. It is interesting that the whole "subshape theory" also works in the relative cases. Even more, it brings a few new phenomena.

\section{The $S^{*}$-EQUivalence}

We hereby apply the previously exhibited "subshape theory" to characterize the Mardešić-Uglešić $S^{*}$-equivalence (see [6] and [7]) as the isomorphisms classification in a certain subshape category.

Recall that two inverse sequences $\boldsymbol{X}, \boldsymbol{Y} \in \mathrm{Ob}(\underline{\mathcal{S}})$ are said to be $S$ equivalent, denoted by $S(\boldsymbol{X})=S(\boldsymbol{Y})$, provided, for every $n \in \mathbb{N}$,

$$
\begin{aligned}
& \left(\forall j_{1}\right)\left(\exists i_{1}\right)\left(\forall i_{1}^{\prime} \geq i_{1}\right)\left(\exists j_{1}^{\prime} \geq j_{1}\right)\left(\forall j_{2} \geq j_{1}^{\prime}\right)\left(\exists i_{2} \geq i_{1}^{\prime}\right) \cdots \\
& \cdots\left(\forall i_{n-1}^{\prime} \geq i_{n-1}\right)\left(\exists j_{n-1}^{\prime} \geq j_{n-1}\right)\left(\forall j_{n} \geq j_{n-1}^{\prime}\right)\left(\exists i_{n} \geq i_{n-1}^{\prime}\right)
\end{aligned}
$$

and there exist mappings $f_{k} \equiv f_{j_{k}}^{n}: X_{i_{k}} \rightarrow Y_{j_{k}}, k=1, \ldots, n$, and $g_{k} \equiv g_{i_{k}^{\prime}}^{n}$ : $Y_{j_{k}^{\prime}} \rightarrow X_{i_{k}^{\prime}}, k=1, \ldots, n-1$, making the following diagram 


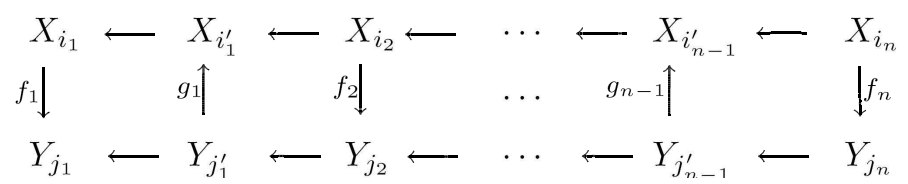

commutative up to homotopy. Two compacta $X$ and $Y$ are said to be $S$ equivalent, denoted by $S(X)=S(Y)$, provided there exist limits $\boldsymbol{p}: X \rightarrow \boldsymbol{X}$ and $\boldsymbol{q}: Y \rightarrow \boldsymbol{Y}$ of inverse sequences consisting of compact ANR's such that $S(\boldsymbol{X})=S(\boldsymbol{Y})$.

Lemma 4.1. Let $\boldsymbol{X}, \boldsymbol{Y} \in \mathrm{Ob}(\underline{\mathcal{S}})$. Then $S(\boldsymbol{X})=S(\boldsymbol{Y})$ if and only if the following two conditions are satisfied:

(1) For every $n \in \mathbb{N}$, there exists a pair $\left(f^{n},\left(F_{j}^{n}\right)_{j \in \mathbb{N}}\right)$ consisting of a strictly increasing function $f^{n}: \mathbb{N} \rightarrow \mathbb{N}$ and, for each $j \in \mathbb{N}$, of a countable family $F_{j}^{n}$ of mappings $f_{\alpha j}^{n}: X_{f^{n}(j)} \rightarrow Y_{j}, \alpha \in A_{j}^{n}$, such that

(i) $(\forall j \in \mathbb{N}) f^{n}(j) \geq j$;

(ii) $\left(\forall j_{1}<\cdots<j_{n}\right.$ in $\left.\mathbb{N}\right)\left(\forall \lambda \in[1, n]_{\mathbb{N}}\right)\left(\exists f_{j_{\lambda}}^{n} \in F_{j_{\lambda}}^{n}\right)$ $\lambda \leq \lambda^{\prime} \Rightarrow f_{j_{\lambda}}^{n} p_{f^{n}\left(j_{\lambda}\right) f^{n}\left(j_{\lambda^{\prime}}\right)} \simeq q_{j_{\lambda} j_{\lambda^{\prime}}} f_{j_{\lambda^{\prime}}}^{n} ;$

(2) For every $n>1$ there exists a pair $\left(g^{n-1},\left(G_{i}^{n-1}\right)_{i \in \mathbb{N}}\right)$ having the properties (i)' and (ii)' analogue to (i) and (ii) respectively, where $g^{n-1}: \mathbb{N} \rightarrow \mathbb{N}$ is increasing, $g_{\beta i}^{n-1}: Y_{g^{n-1}(i)} \rightarrow X_{i}$ is a mapping, $\beta \in B_{i}^{n-1}$, and

(iii) $\left(\forall j_{1}\right)\left(\forall i_{1} \geq f^{n}\left(j_{1}\right)\right)\left(\forall j_{2} \geq g^{n-1}\left(i_{1}\right)\right) \cdots\left(\forall j_{n} \geq f^{n}\left(j_{n-1}\right)\right)$ there exist mappings $f_{j_{1}}^{n} \in F_{j_{1}}^{n}, \ldots, f_{j_{n}}^{n} \in F_{j_{n}}^{n}, g_{i_{1}}^{n-1} \in G_{i_{1}}^{n-1}, \ldots$, $g_{i_{n-1}}^{n-1} \in G_{i_{n-1}}^{n-1}$ such that the corresponding diagram

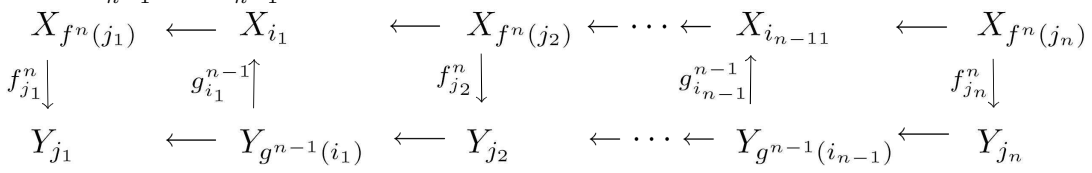
commutes up to homotopy.

Proof. Let $S(\boldsymbol{X})=S(\boldsymbol{Y})$. First, we may assume that, for each $n \in \mathbb{N}$, a choice of indices in the appropriate condition is strictly increasing and that $i_{k}\left(j_{k}\right)>j_{k}, j_{k} \in \mathbb{N}, k=1, \ldots, n$. Thus, if $n=1$, the appropriate condition of $S(\boldsymbol{X})=S(\boldsymbol{Y})$ implies that for every $j \in \mathbb{N}$ there exist an $i \in \mathbb{N}, i>j$, and a mapping $f_{j}: X_{i} \rightarrow Y_{j}$. By moving indices, inductively, one easily obtains a strictly increasing function $f^{1}: \mathbb{N} \rightarrow \mathbb{N}$ satisfying condition (i) as well as, for each $j \in \mathbb{N}$, the singleton family $F_{j}^{1}=\left\{f_{j}^{1}\right\}, f_{j}^{1}=f_{j}: X_{f^{1}(j)} \rightarrow Y_{j}$. Since there is no commutativity condition, $\left(f^{1},\left(F_{j}^{1}\right)\right)$ is a desired pair. Let $n \geq 2$. Consider first the simplest case $n=2$ by using the corresponding condition of $S(\boldsymbol{X})=S(\boldsymbol{Y})$. First, we shall inductively construct a desired strictly increasing function $f^{2}: \mathbb{N} \rightarrow \mathbb{N}$. For $j_{1}=1$,

$$
\left(\exists i_{1} \geq 1\right)\left(\forall i_{1}^{\prime} \geq i_{1}\right)\left(\exists j_{1}^{\prime} \geq i_{1}^{\prime}\right)\left(\forall j_{2} \geq j_{1}^{\prime}\right)\left(\exists i_{2} \geq j_{2}\right)
$$


and there exist mappings $f_{1}: X_{i_{1}} \rightarrow Y_{1}, f_{2}: X_{i_{2}} \rightarrow Y_{j_{2}}, g_{1}: Y_{j_{1}^{\prime}} \rightarrow X_{i_{1}^{\prime}}$ such that the corresponding diagram commutes up to homotopy. The index $i_{1}$ depends only on $j_{1}=1$ (and on $n=2$, of course). Thus, put $f^{2}(1)=i_{1}(1)$. Assume that $f^{2}(1)<\cdots<f^{2}(j)$ are defined according to (i). As before, for $j_{1}=j+1$,

$$
\left(\exists i_{1} \geq j+1\right)\left(\forall i_{1}^{\prime} \geq i_{1}\right)\left(\exists j_{1}^{\prime} \geq i_{1}^{\prime}\right)\left(\forall j_{2} \geq j_{1}^{\prime}\right)\left(\exists i_{2} \geq j_{2}\right)
$$

and there exist mappings $f_{1}: X_{i_{1}} \rightarrow Y_{1}, f_{2}: X_{i_{2}} \rightarrow Y_{j_{2}}, g_{1}: Y_{j_{1}^{\prime}} \rightarrow X_{i_{1}^{\prime}}$ such that the corresponding diagram commutes up to homotopy. The index $i_{1}$ depends only on $j_{1}=j+1$. Notice that in each of the previous cases, i.e. $j_{1} \in[1, j]_{\mathbb{N}}$, the index $j_{2}=j+1$ may occur at most finitely many times. Therefore, in all those cases, $j_{2}=j+1$ occurs only finitely many times. Denote the set of all the corresponding indices $i_{2}$ by $I\left(j_{1}, j_{2}=j+1\right)$. Put

$$
f^{2}(j+1)=\max \left\{f^{2}(j)+1, i_{2}^{*}, i_{1}(j+1)\right\},
$$

where $i_{2}^{*}=\max I\left(j_{1}, j_{2}=j+1\right)$. Let $F_{1}^{2}$ consist of all the occurring mappings $f_{1 \alpha}^{2}: X_{f^{2}(1)} \rightarrow Y_{1}, \alpha \in A_{1}^{2}$. If $j>1$, let $F_{j}^{2}$ consist of all the mappings $f_{j \alpha}^{2}: X_{f^{2}(j)} \rightarrow Y_{j}, \alpha \in A_{j}^{2}$, which are the compositions of the occurring mappings with the appropriate bonding mappings. Properties (i) and (ii) for the pair $\left(f^{2},\left(F_{j}^{2}\right)\right)$ follow by the condition of $S(\boldsymbol{X})=S(\boldsymbol{Y})$ for $n=2$.

To clarify the general case, let us carefully explain the case $n=3$. Observe that each $j \in \mathbb{N}$ occurs in a $\boldsymbol{j}^{3}=\left(j_{1}, j_{2}, j_{3}\right)$ as one of its coordinate. If $j=j_{3}$ then there are only finitely many triples containing $j$, and thus, there are only finitely many corresponding indices $i(j)$. If $j=j_{2}$ then there are infinitely many triples containing $j$; only finitely many of them (by varying $j_{1}$ ) require different choices of $i(j)$. If $j=j_{1}$ then there are infinitely many triples containing $j$; however, in these cases $i(j)$ depends only of $j$, so it may be a unique index. Therefore, for each $j \in \mathbb{N}$, there exists a finite set $I_{j}\left(j^{3}\right) \subseteq \mathbb{N}$, $j \in \boldsymbol{j}^{3}$ and $\boldsymbol{j}^{3} \in \boldsymbol{J}(3)$, containing all the indices $i(j)$ which comes from the condition $S(\boldsymbol{X})=S(\boldsymbol{Y})$ for $n=3$. Quite similarly to the previous case, one can inductively construct a desired pair $\left(f^{3},\left(F_{j}^{3}\right)\right)$. To conclude the proof of the existence of every pair $\left(f^{n},\left(F_{j}^{n}\right)\right), n \in \mathbb{N}$, it suffices to observe that our main argument (i.e. for every $j \in \mathbb{N}$, the corresponding set $I_{j}\left(j^{n}\right), j \in j^{n}$ and $\boldsymbol{j}^{n} \in \boldsymbol{J}(\mathrm{n})$, is finite), holds in general.

Let us now construct a desired pair $\left(g^{n-1},\left(G_{i}^{n-1}\right)\right)$, whenever $n>1$. First, consider the case $n=2$. Denote $f^{2}(1)=i_{1}$. By $S(\boldsymbol{X})=S(\boldsymbol{Y})$ for $n=2$ and our construction of $\left(f^{2},\left(F_{j}^{2}\right)\right)$, for $j_{1}=1$,

$$
\left(\exists i_{1}=f^{2}(1) \geq 1\right)\left(\forall i_{1}^{\prime} \geq i_{1}\right)\left(\exists j_{1}^{\prime} \geq i_{1}^{\prime}\right)\left(\forall j_{2} \geq j_{1}^{\prime}\right)\left(\exists i_{2} \geq j_{2}\right)
$$

and there exist mappings $f_{1}: X_{f^{2}(1)} \rightarrow Y_{1}, f_{2}: X_{i_{2}} \rightarrow Y_{j_{2}}, g_{1}: Y_{j_{1}^{\prime}} \rightarrow X_{i_{1}^{\prime}}$ such that the corresponding diagram commutes up to homotopy. Notice that for $i_{1}^{\prime}=f^{2}(1)$, the index $j_{1}^{\prime}\left(f^{2}(1)\right)$ is a unique one. Put $g^{1}(i)=j_{1}^{\prime}\left(f^{2}(1)\right)$ for every $i \in\left[1, f^{2}(1)\right]_{\mathbb{N}}$. For $i>f^{2}(1)$, we may proceed inductively as in the 
first part of the proof, since for every $i \in \mathbb{N}$, the set $J_{i}\left(i^{2}\right) \subseteq \mathbb{N}, i \in i^{2}$ and $\boldsymbol{i}^{2} \in \boldsymbol{J}(2)$, (of all the indices $j^{\prime}(i)$ coming from the condition $S(\boldsymbol{X})=S(\boldsymbol{Y})$ for $n=2)$ is finite. Thus, we can obtain a desired pair $\left(g^{1},\left(G_{i}^{1}\right)\right)$. Condition (iii) follows by construction and the condition of $S(\boldsymbol{X})=S(\boldsymbol{Y})$ for $n=2$.

Since the main argument (every $J_{i}\left(\boldsymbol{i}^{n}\right)$ is a finite set) holds in general, the conclusion follows. The converse, i.e. that conditions (1) and (2) imply $S(\boldsymbol{X})=S(\boldsymbol{Y})$, is obvious.

EXAmPle 4.2. Let $S(\boldsymbol{X})=S(\boldsymbol{Y})$. Then, according to Lemma 4.1, for every $k \in \mathbb{N}$ there exists a pair $\left(f^{k},\left(F_{j}^{k}\right)_{j \in \mathbb{N}}\right)$. Given an $n \in \mathbb{N}$ and a $\boldsymbol{j}^{n} \in \boldsymbol{J}(n)$, one can easily obtain an $n$-ladder $\boldsymbol{f}_{\boldsymbol{j}^{n}}^{k}: \boldsymbol{X} \rightarrow \boldsymbol{Y}$ by means of $\left(f^{k},\left(F_{j}^{k}\right)_{j \in \mathbb{N}}\right)$, where $k \geq n$ is chosen arbitrarily. Moreover, a $\lambda$-block of $\boldsymbol{f}_{\boldsymbol{j}^{n}}^{k}$ is not empty whenever $j_{\lambda+1}-j_{\lambda}$ is sufficiently large. However, an easy examination shows that, in general, the family $\left(\boldsymbol{f}_{\boldsymbol{j}^{n}}^{k}\right), \boldsymbol{j}^{n} \in \boldsymbol{J}(n)$, is not an $n$ hyperladder. Namely, in general, both conditions $\left(S(n, m)_{1}\right)$ and $\left(S(n, m)_{2}\right)$ cannot be fulfilled. The obstruction also remains if one defines the family $\left(\boldsymbol{f}_{\boldsymbol{j}^{n}}\right), \boldsymbol{j}^{n} \in \boldsymbol{J}(n)$, by using any or all $k \geq n$.

If $S(\boldsymbol{X})=S(\boldsymbol{Y})$ can be obtained so that the choice of indices $i_{k}$ and $j_{k}^{\prime}$ does not depend on a given $n \in \mathbb{N}$, then the (formally) stronger relation $S^{*}(\boldsymbol{X})=S^{*}(\boldsymbol{Y})$, as well as $S^{*}(X)=S^{*}(Y)$, occurs. According to Lemma 4.1 , it can be characterized by conditions (1) and (2) such that, in addition, $f^{n}=f$ for every $n$ and $g^{n-1}=g$ for every $n>1$. It is now an easy exercise to exhibit the next lemma by means of Lemma 4.1.

LEMMA 4.3. $S^{*}(\boldsymbol{X})=S^{*}(\boldsymbol{Y})$ if and only if there exists a pair of strictly increasing sequences $\left(u_{k}\right)$ and $\left(v_{k}\right)$ in $\mathbb{N}, v_{k} \leq u_{k} \leq v_{k+1}$ for every $k \in \mathbb{N}$, such that, for every $n \in \mathbb{N}$ and every $k \in \mathbb{N}$, there exist mappings $f_{l}^{n} \equiv f_{v_{k+l-1}}^{n}$ : $X_{u_{k+l-1}} \rightarrow Y_{v_{k+l-1}}, l=1, \ldots, n$, and $g_{l}^{n} \equiv g_{u_{k+l-1}}^{n}: Y_{v_{k+l}} \rightarrow X_{u_{k+l-1}}, l=$ $1, \ldots, n-1$, making the diagram

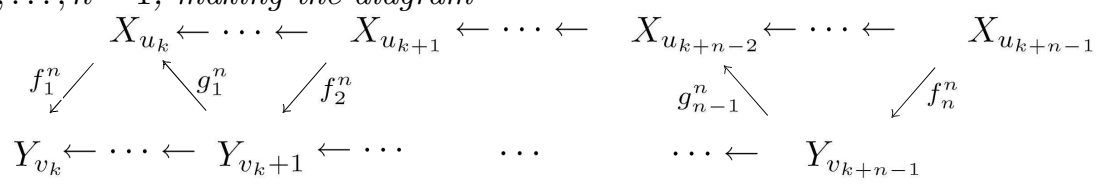

homotopy commutative.

S. Mardešić and the first named author (see [7]) constructed a certain category $\underline{\mathcal{S}}^{*}$ (and the functor $\underline{S}^{*}: \mathcal{S} h \rightarrow \underline{\mathcal{S}}^{*}$ ), such that $\boldsymbol{X} \cong \boldsymbol{Y}$ in $\underline{\mathcal{S}}^{*}$ if and only if $S^{*}(\boldsymbol{X})=S^{*}(\boldsymbol{Y})$. Further, in the corresponding category $\mathcal{S}^{*}$ on compacta, $X \cong Y$ in $\mathcal{S}^{*}$ if and only if $S^{*}(X)=S^{*}(Y)$.

We first prove that the $S^{*}$-equivalence implies an isomorphism in the sequence subshape category $\underline{\mathcal{S}}(\mathbb{N})$. 
Theorem 4.4. Let $\boldsymbol{X}, \boldsymbol{Y} \in \mathrm{Ob}(\underline{\mathcal{S}})$. If $S^{*}(\boldsymbol{X})=S^{*}(\boldsymbol{Y})$ then $\boldsymbol{X} \cong \boldsymbol{Y}$ in the category $\underline{\mathcal{S}}(\mathbb{N})$ and, consequently, in every category $\underline{\mathcal{S}}(n), n \in \mathbb{N}$. The same holds for $X, Y \in \mathrm{Ob}(c \mathcal{M})$ and the categories $\mathcal{S}(\mathbb{N})$ and $\mathcal{S}(n), n \in \mathbb{N}$.

Proof. Let $S^{*}(\boldsymbol{X})=S^{*}(\boldsymbol{Y})$. We have to construct, for every $n \in \mathbb{N}$, a pair of morphisms $\boldsymbol{F}_{n}=\left[F_{n}\right] \in \underline{\mathcal{S}}(n)(\boldsymbol{X}, \boldsymbol{Y}), \boldsymbol{G}_{n}=\left[G_{n}\right] \in \underline{\mathcal{S}}(n)(\boldsymbol{Y}, \boldsymbol{X})$ such that $\boldsymbol{G}_{n} \boldsymbol{F}_{n}=\mathbf{1}_{\boldsymbol{X} n}$ and $\boldsymbol{F}_{n} \boldsymbol{G}_{n}=\mathbf{1}_{\boldsymbol{Y} n}$, i.e. $G_{n} F_{n} \simeq 1_{\boldsymbol{X} n}$ and $F_{n} G_{n} \simeq 1_{\boldsymbol{Y} n}$ for every pair of the representatives. By Lemma 4.3, for every $n \in \mathbb{N}$, every $\boldsymbol{j}^{n} \in \boldsymbol{J}(n)$ determines two (finite) sets of indices, belonging to the existing sequences,

$$
\begin{aligned}
V\left(\boldsymbol{j}^{n}\right) & =\left\{v_{k^{\prime}} \mid v_{k^{\prime}} \in\left[j_{1}, j_{n+1}-1\right]_{\mathbb{N}}, k^{\prime} \geq k, v_{k-1}<j_{1}, u_{k^{\prime}}<j_{n+1}\right\}, \\
U\left(\boldsymbol{j}^{n}\right) & \left.=\left\{u_{k^{\prime}} \mid u_{k^{\prime}} \in\left[j_{1}, j_{n+1}-1\right]_{\mathbb{N}}\right\}, k^{\prime} \geq k, v_{k-1}<j_{1}\right\} .
\end{aligned}
$$

Then $\left|V\left(\boldsymbol{j}^{n}\right)\right|=\left|U\left(\boldsymbol{j}^{n}\right)\right| \equiv n^{\prime} \in \mathbb{N}$ (depending only on $j_{1}$ and $j_{n+1}$ ). By considering the corresponding diagram of Lemma 4.3, let us denote, for every $\lambda \in[1, n]_{\mathbb{N}}$,

$$
v_{k_{\lambda}}, v_{k_{\lambda}+1}, \ldots, v_{k_{\lambda}+r_{\lambda}} \in V\left(j^{n}\right) \cap\left[j_{\lambda}, j_{\lambda+1}-1\right]_{\mathbb{N}},
$$

i.e. $u_{k_{\lambda}+r_{\lambda}}<j_{\lambda+1}-1$ and $u_{k_{\lambda}+r_{\lambda}+1} \geq j_{\lambda+1}$. Then we define the $n$-ladder $\boldsymbol{f}_{\boldsymbol{j}^{n}}: \boldsymbol{X} \rightarrow \boldsymbol{Y}$ by putting

$$
\begin{gathered}
\left(\forall \lambda \in[1, n]_{\mathbb{N}}\right)\left(\forall s_{\lambda} \in\left[0, r_{\lambda}\right]_{\mathbb{N}_{0}}\right)\left(\forall j \in\left[v_{k_{\lambda}+s_{\lambda}-1}+1, v_{k_{\lambda}+s_{\lambda}}\right]_{\mathbb{N}}\right) \\
f_{j}=q_{j v_{k_{\lambda}+s_{\lambda}}} f_{\lambda, s_{\lambda}+1}^{n^{\prime}},
\end{gathered}
$$

where $v_{k_{\lambda}-1} \equiv j_{\lambda}-1$ and $f_{\lambda, s_{\lambda}+1}^{n^{\prime}} \equiv f_{v_{k_{\lambda}+s_{\lambda}+1}}^{n^{\prime}}: X_{u_{k_{\lambda}+s_{\lambda}}} \rightarrow Y_{v_{k_{\lambda}+s_{\lambda}}}$.

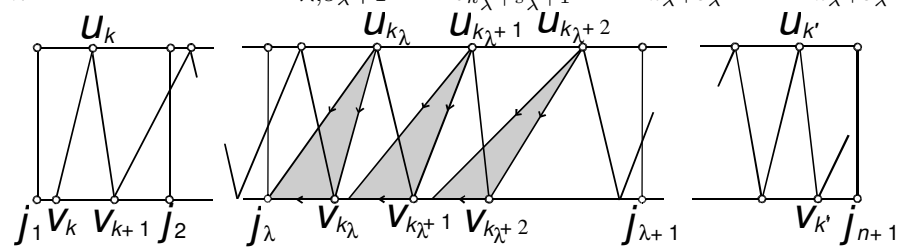

One can easily verify that $F_{n}=\left(\boldsymbol{f}_{\boldsymbol{j}^{n}}\right)$, indexed by all $\boldsymbol{j}^{n} \in \boldsymbol{J}(n)$, is an $n$ hyperladder of $\boldsymbol{X}$ to $\boldsymbol{Y}$. (Given a $j_{\lambda}^{\prime} \geq j_{\lambda}$, choose the minimal $v_{k} \equiv v_{k_{\lambda}} \geq j_{\lambda}^{\prime}$ and put $i^{\lambda}=u_{k_{\lambda}}$.) The $n$-hyperladder $G_{n}=\left(\boldsymbol{g}_{\boldsymbol{i}^{n}}\right)$ of $\boldsymbol{Y}$ to $\boldsymbol{X}$ is defined in the same way by means of $U\left(\boldsymbol{j}^{n}\right)=V\left(\boldsymbol{j}^{n}\right)$ and the mappings $g_{u_{k_{\lambda}+s_{\lambda}+1}}^{n^{\prime}}$.

Let us prove that $G_{n} F_{n} \simeq 1_{\boldsymbol{X} n}$ and $F_{n} G_{n} \simeq 1_{\boldsymbol{Y} n}$. Let $n \stackrel{\mathbb{N}}{\text { and }}$ let $m \leq n$. Then, for every $j_{1} \in \mathbb{N}$ and every $j_{1}^{\prime} \geq j_{1}$ there exists a minimal $v_{k_{1}} \geq j_{1}^{\prime}$; put $j_{*}^{1}=v_{k_{1}+1} ; \ldots$; for every $j_{m}>j_{*}^{m-1}$ and every $j_{m}^{\prime} \geq j_{m}$ there exists a minimal $v_{k_{m}} \geq j_{m}^{\prime}$; put $j_{*}^{m}=v_{k_{m}+1}$; let $j_{m+1}>j_{*}^{m}, j_{m+2}>j_{m+1}$, $\ldots, j_{n+1}>j^{n}$. 

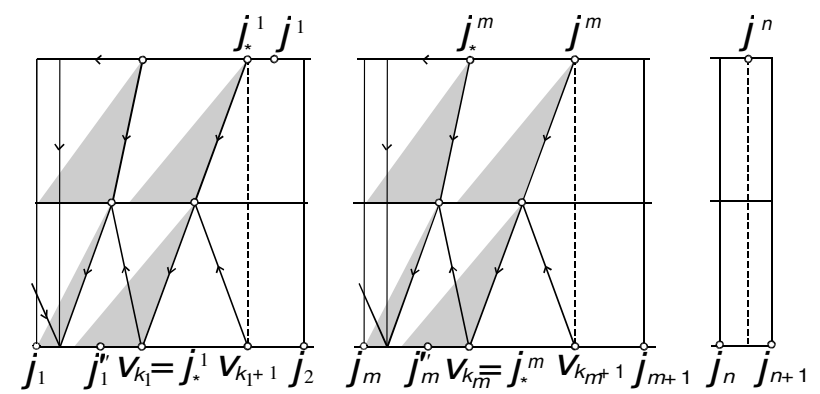

Then condition $(H(n, m))$ for $F_{n} G_{n}$ and $1_{\boldsymbol{Y} n}$, i.e. the $m$-homotopy relation $\boldsymbol{f}_{\boldsymbol{j}^{n}} \boldsymbol{g}_{\boldsymbol{j}^{n}} \simeq_{m} \mathbf{1}_{\boldsymbol{Y} \boldsymbol{j}^{n}}$ holds by construction of the $n$-ladders. Therefore, $F_{n} G_{n} \simeq 1_{\boldsymbol{Y} n}$. Further, for every $i_{1} \in \mathbb{N}$ and every $i_{1}^{\prime} \geq i_{1}$ there exists a minimal $u_{k_{1}} \geq i_{1}^{\prime}$; put $i_{*}^{1}=u_{k_{1}+1} ; \ldots$; for every $i_{m}>i_{*}^{m-1}$ and every $i_{m}^{\prime} \geq i_{m}$ there exists a minimal $u_{k_{m}} \geq i_{m}^{\prime}$; put $i_{*}^{m}=u_{k_{m}+1}$; let $i_{m+1}>i_{*}^{m}$, $i_{m+2}>i_{m+1}, \ldots, i_{n+1}>i_{n}$.

Then condition $(H(n, m))$ for $G_{n} F_{n}$ and $1_{\boldsymbol{X} n}$, i.e. the $m$-homotopy relation $\boldsymbol{g}_{\boldsymbol{i}^{n}} \boldsymbol{f}_{\boldsymbol{i}^{n}} \simeq_{m} \mathbf{1}_{\boldsymbol{X} \boldsymbol{i}^{n}}$ holds by construction of the $n$-ladders. Therefore, $G_{n} F_{n} \simeq 1_{\boldsymbol{X} n}$. The proof of the rest of the theorem is quite similar.

An $n$-hyperladder $F_{n}=\left(\boldsymbol{f}_{\boldsymbol{j}^{n}}\right): \boldsymbol{X} \rightarrow \boldsymbol{Y}, \boldsymbol{j}^{n} \in \boldsymbol{J}(n), n \in \mathbb{N} \cup\{\omega\}$, is said to be uniform and is denoted by $F_{n}=\left(f_{n}, \boldsymbol{f}_{\boldsymbol{j}^{n}}\right)$, provided there exists a strictly increasing function $f_{n}: \mathbb{N} \rightarrow \mathbb{N}$ such that the index function of every $n$-ladder $\boldsymbol{f}_{\boldsymbol{j}^{n}} \in F_{n}$ is the appropriate restriction of $f_{n}$. If $G_{n}=\left(g_{n}, \boldsymbol{g}_{\boldsymbol{k}^{n}}\right): \boldsymbol{Y} \rightarrow \boldsymbol{Z}$ is an other uniform $n$-hyperladder, then the composition

$$
G_{n} F_{n}=\left(f_{n} g_{n}, \boldsymbol{g}_{\boldsymbol{k}^{n}} \boldsymbol{f}_{\boldsymbol{k}^{n}}\right): \boldsymbol{X} \rightarrow \boldsymbol{Z}
$$

is a uniform $n$-hyperladder. Further, each identity $n$-hyperladder $1_{\boldsymbol{X} n}=$ $\left(1_{\mathbb{N}}, \mathbf{1}_{\boldsymbol{X} \boldsymbol{i}^{n}}\right), \boldsymbol{X} \in \mathrm{Ob} \mathcal{S}$, is uniform. Thus, there exists the corresponding subcategory $\underline{\mathcal{L}}_{0}(n) \subseteq \underline{\mathcal{L}}(n)$ determined by all the uniform $n$-hyperladders. The appropriate quotient (homotopy) category is denoted by $\underline{\mathcal{S}}_{0}(n)$. Let $\underline{\mathcal{S}}_{*}(n)$ be the largest subcategory of $\underline{\mathcal{S}}(n)$ such that every morphism $\boldsymbol{F}_{n}: \underline{X}_{\rightarrow} \boldsymbol{Y}$ of $\underline{\mathcal{S}}_{*}(n)$ admits a uniform representative $F_{n}=\left(f_{n}, \boldsymbol{f}_{\boldsymbol{j}^{n}}\right)$. Clearly, $\underline{\mathcal{S}}_{0}(n) \subseteq$ $\underline{\mathcal{S}}_{*}(n)$ and, for the corresponding sequence categories, $\underline{\mathcal{S}}_{0}(\mathbb{N}) \subseteq \underline{\mathcal{S}}_{*}(\mathbb{N})$.

Lemma 4.5. If $\boldsymbol{X} \cong \boldsymbol{Y}$ in $\underline{\mathcal{S}}_{*}(\mathbb{N})$, then $S(\boldsymbol{X})=S(\boldsymbol{Y})$. Consequently, if $X \cong Y$ in $\mathcal{S}_{*}(\mathbb{N})$, then $S(X)=S(Y)$.

Proof. Let $\boldsymbol{X} \cong \boldsymbol{Y}$ in $\underline{\mathcal{S}}_{*}(\mathbb{N})$. Then there exists a pair of sequences $\left(F_{k}\right),\left(G_{k}\right)$ such that, for every $k \in \mathbb{N}, F_{k}=\left(f_{k}, \boldsymbol{f}_{\boldsymbol{j}^{k}}\right) \in \underline{\mathcal{L}}_{0}(k)(\boldsymbol{X}, \boldsymbol{Y}), G_{k}=$ $\left(g_{k}, \boldsymbol{g}_{\boldsymbol{i}^{k}}\right) \in \underline{\mathcal{L}}_{0}(k)(\boldsymbol{Y}, \boldsymbol{X}), G_{k} F_{k} \simeq 1_{\boldsymbol{X} k}$ and $F_{k} G_{k} \simeq 1_{\boldsymbol{Y} k}$. This also means that $\boldsymbol{X} \cong \boldsymbol{Y}$ in each $\underline{\mathcal{S}}_{*}(k), k \in \mathbb{N}$. We have to exhibit, for a given $n \in \mathbb{N}$, a homotopy commutative diagram corresponding to $S(\boldsymbol{X})=S(\boldsymbol{Y})$. The case $n=1$ is trivial. Consider the case $n=2$. Let us denote $U_{1}=\left(u_{1}, \boldsymbol{u}_{\boldsymbol{s}^{1}}\right)=G_{1} F_{1}$ and $V_{1}=\left(v_{1}, \boldsymbol{v}_{\boldsymbol{t}^{1}}\right)=F_{1} G_{1}$. Then $u_{1}=f_{1} g_{1}, \boldsymbol{u}_{\boldsymbol{s}^{1}}=\boldsymbol{g}_{\boldsymbol{s}^{1}} \boldsymbol{f}_{\boldsymbol{s}^{1}}, \boldsymbol{s}^{1} \in \boldsymbol{J}(1)$, and $v_{1}=g_{1} f_{1}, \boldsymbol{v}_{\boldsymbol{t}^{1}}=\boldsymbol{f}_{\boldsymbol{t}^{1}} \boldsymbol{g}_{\boldsymbol{t}^{1}}, \boldsymbol{t}^{1} \in \boldsymbol{J}(1)$. 
Now, for every $j_{1} \in \mathbb{N}$, put $t_{1}=s_{1}=j_{1}$ and $i_{1}=f_{1}\left(t_{1}\right)$. Let $i_{1}^{\prime} \geq i_{1}$. Since $f_{1}$ strictly increases, there exists a $t_{1}^{\prime} \geq t_{1}$ such that $f_{1}\left(t_{1}^{\prime}\right) \geq i_{1}^{\prime}$. By $V_{1} \simeq 1_{Y 1}$, for $t_{1}$ and $t_{1}^{\prime}$ there exists a $t_{*}^{1} \geq t_{1}^{\prime}$. Put $j_{1}^{\prime}=t_{*}^{1}$ and let $j_{2} \geq j_{1}^{\prime}$. Since $g_{1}$ strictly increases, there exists an $s_{1}^{\prime} \geq f_{1}\left(t_{1}^{\prime}\right)$ such that $g_{1}\left(s_{1}^{\prime}\right) \geq j_{2}$. By $U_{1} \simeq 1_{X 1}$, for $s_{1}$ and $s_{1}^{\prime}$, there exists an $s_{*}^{1} \geq s_{1}^{\prime}$. Put $i_{2}=s_{*}^{1}$, and choose an $s_{2}=t_{2} \geq g_{1}\left(i_{2}\right)+1$. Then $\boldsymbol{s}^{1}=\boldsymbol{t}^{1}$ and corresponding 1-ladders $\boldsymbol{f}_{\boldsymbol{t}^{1}}: \boldsymbol{X} \rightarrow \boldsymbol{Y}$ and $\boldsymbol{g}_{s^{1}}: \boldsymbol{Y} \rightarrow \boldsymbol{X}$ yield the mappings

$$
\begin{aligned}
f_{1}^{2} & =f_{t_{1}}: X_{i_{1}}=X_{f_{1}\left(t_{1}\right)} \rightarrow Y_{t_{1}}=Y_{j_{1}}, \\
g_{1}^{2} & =g_{i_{1}^{\prime}} q_{g_{1}\left(i_{1}^{\prime}\right) t_{*}^{1}}: Y_{j_{1}^{\prime}}=Y_{t_{*}^{1}} \rightarrow X_{i_{1}^{\prime}}, \\
f_{2}^{2} & =f_{j_{2}} p_{f_{1}\left(j_{2}\right) s_{*}^{1}}: X_{i_{2}}=X_{s_{*}^{1}} \rightarrow Y_{j_{2}} .
\end{aligned}
$$

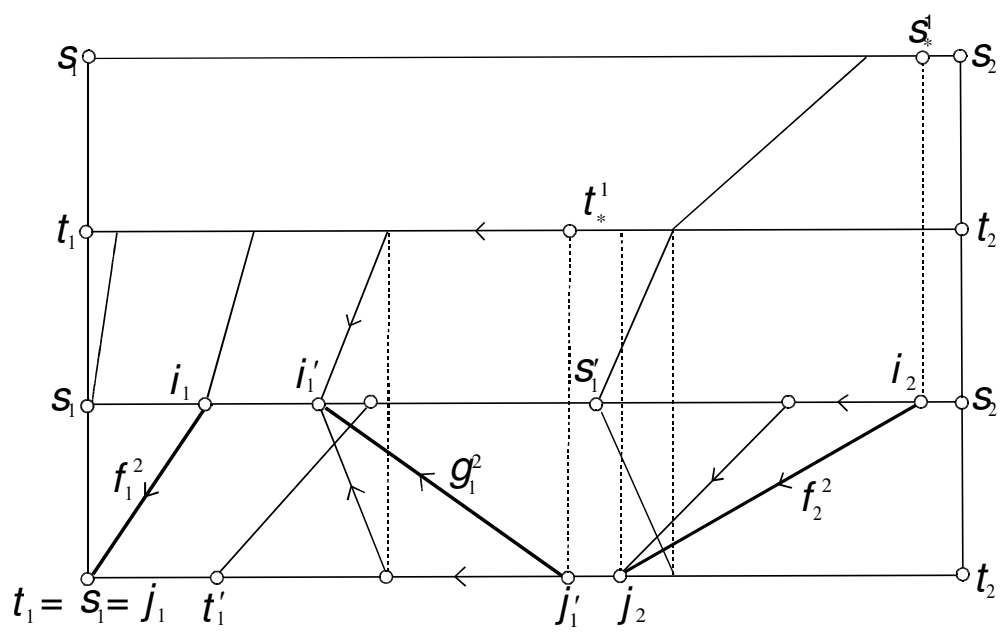

Clearly, the mappings $f_{t_{1}}$ and $f_{j_{2}}$ belong to $f_{t^{1}}$, while the mapping $g_{i_{1}^{\prime}}$ belongs to $\boldsymbol{g}_{\boldsymbol{s}^{1}}, \boldsymbol{s}^{1}=\boldsymbol{t}^{1}$. To obtain the homotopy commutative diagram which corresponds to $S(\boldsymbol{X})=S(\boldsymbol{Y})$ in the case $n=2$ follows as straightforward by the homotopy relations $\boldsymbol{f}_{\boldsymbol{t}^{1}} \boldsymbol{g}_{\boldsymbol{t}^{1}} \simeq \mathbf{1}_{\boldsymbol{Y} \boldsymbol{t}^{1}}$ and $\boldsymbol{g}_{\boldsymbol{s}^{1}} \boldsymbol{f}_{\boldsymbol{s}^{1}} \simeq \mathbf{1}_{\boldsymbol{X} \boldsymbol{s}^{1}}$ of the composite 1-ladders.

If $n>2$, the procedure of the construction is similar. For instance, if $n=3$, we use $F_{2}$ and $G_{2}$. More precisely (after $i_{2}$ is chosen), for every $i_{2}^{\prime} \geq i_{2}=s_{*}^{1}$, put $s_{2}=t_{2}=\max \left\{g_{2}\left(i_{2}\right)+1, i_{2}^{\prime}\right\}$ and proceed as before. The desired mappings $f_{1}^{3}, g_{1}^{3}$ and $f_{2}^{3}$ occur in the first block, while $g_{2}^{3}$ and $f_{3}^{3}$ occur in the second block.

Let $\underline{\mathcal{S}}^{*}(\mathbb{N}) \subseteq \underline{\mathcal{S}}_{*}(\mathbb{N})$ be the largest subcategory such that every morphism $\boldsymbol{F}=\left(\boldsymbol{F}_{n}\right) \in \underline{\mathcal{S}}^{*}(\mathbb{N})(\boldsymbol{X}, \boldsymbol{Y})$ admits a representative $\left(F_{n}\right), F_{n}=\left(f_{n}, \boldsymbol{f}^{\underline{j}}{ }_{n}\right) \in$ $\underline{\mathcal{S}}_{0}(\mathbb{N})(\boldsymbol{X}, \boldsymbol{Y})$, such that there exists a function $f: \mathbb{N} \rightarrow \mathbb{N}$ satisfying $f_{n}=f$, for every $n \in \mathbb{N}$. Then Lemma 4.5 and its proof yield the next corollary. 
Corollary 4.6. If $\boldsymbol{X} \cong \boldsymbol{Y}$ in $\underline{\mathcal{S}}^{*}(\mathbb{N})$, then $S^{*}(\boldsymbol{X})=S^{*}(\boldsymbol{Y})$. Consequently, if $X \cong Y$ in $\mathcal{S}^{*}(\mathbb{N})$, then $S^{*}(X)=S^{*}(Y)$.

Proof. Since the sequences $\left(F_{k}\right)$ and $\left(G_{k}\right)$ admit the unique index functions $f$ and $g$, respectively, the choice of indices for $S(\boldsymbol{X})=S(\boldsymbol{Y})$ does not depend on any $n \in \mathbb{N}$. The conclusion follows, i.e. in this case $S(\boldsymbol{X})=S(\boldsymbol{Y})$ is actually $S^{*}(\boldsymbol{X})=S^{*}(\boldsymbol{Y})$.

We shall now prove that the converse also holds.

Theorem 4.7. $S^{*}(\boldsymbol{X})=S^{*}(\boldsymbol{Y})$ if and only if $\boldsymbol{X} \cong \boldsymbol{Y}$ in the subcategory $\underline{\mathcal{S}}^{*}(\mathbb{N}) \subseteq \underline{\mathcal{S}}(\mathbb{N})$. Consequently, for every pair $X, Y$ of compacta, $S^{*}(X)=$ $S^{*}(Y)$ if and only if $X \cong Y$ in the subcategory $\mathcal{S}^{*}(\mathbb{N}) \subseteq \mathcal{S}(\mathbb{N})$.

Proof. The subcategory $\underline{\mathcal{S}}^{*}(\mathbb{N}) \subseteq \underline{\mathcal{S}}(\mathbb{N})$ is already constructed. The sufficiency holds by Corollary 4.6. Let us prove the necessity part. First, notice that the proof of Theorem 4.4 may not be used since we now need the uniform $n$-hyperladders which must have the same index function for the whole sequence. To avoid this difficulty, we shall apply Lemma 4.1 adapted to the $S^{*}$-equivalence. In that case, the pairs $\left(f^{n},\left(F_{j}^{n}\right)_{j \in \mathbb{N}}\right)$ and $\left(g^{n-1},\left(G_{i}^{n-1}\right)_{i \in \mathbb{N}}\right)$ have this additional property:

$$
(\forall n \in \mathbb{N}) f^{n}=f \text { and }(\forall n>1) g^{n-1}=g .
$$

Let $n \in \mathbb{N}$ and $\boldsymbol{j}^{n} \in \boldsymbol{J}(n)$. Then the pair $\left(f,\left(F_{j}^{k}\right)_{j \in \mathbb{N}}\right), k=j_{n+1}-j_{1}$, admits a $k$-tuple $\left(f_{j_{1}}^{k}, \ldots, f_{j_{n+1}-1}^{k}\right), f_{j}^{k} \in F_{j}^{k}, j \in\left[j_{1}, j_{n+1}-1\right]_{\mathbb{N}}$, such that

$$
\left(\forall j, j^{\prime} \in\left[j_{1}, j_{n+1}-1\right]_{\mathbb{N}}\right) j \leq j^{\prime} \Rightarrow\left[f_{j}^{k}\right]\left[p_{f(j) f\left(j^{\prime}\right)}\right]=\left[q_{j j^{\prime}}\right]\left[f_{j^{\prime}}^{k}\right] .
$$

Let the $n$-ladder $\boldsymbol{f}_{\boldsymbol{j}^{n}}: \boldsymbol{X} \rightarrow \boldsymbol{Y}$ be induced by $\left(f_{j_{1}}^{k}, \ldots, f_{j_{n+1}-1}^{k}\right)$, i.e. for every $\lambda \in[1, n]_{\mathbb{N}}$ and every $j \in\left[j_{\lambda}, j_{\lambda+1}-1\right]_{\mathbb{N}}$, the homotopy class of the mapping $f_{j}^{k}$ belongs to (the $\lambda$-th block of) $\boldsymbol{f}_{\boldsymbol{j}^{n}}$, whenever $f(j)<j_{\lambda+1}$. Then, one can easily verify that the corresponding family $F_{n}=\left(\boldsymbol{f}_{\boldsymbol{j}^{n}}\right), \boldsymbol{j}^{n} \in \boldsymbol{J}(n)$, is an $n$-hyperladder of $\boldsymbol{X}$ to $\boldsymbol{Y}$. Moreover,

$$
F_{n}=\left(\boldsymbol{f}_{\boldsymbol{j}^{n}}\right)=\left(f_{n}=f, \boldsymbol{f}_{\boldsymbol{j}^{n}}\right), \boldsymbol{j}^{n} \in \boldsymbol{J}(n),
$$

belongs to $\underline{\mathcal{S}}_{0}(n)(\boldsymbol{X}, \boldsymbol{Y})$ and in the sequence $\left(F_{n}\right)$ all the $F_{n}$ 's have the same index function $f$. Let the sequence $\left(G_{n}\right)$ of $n$-hyperladders $G_{n}=\left(g_{n}=\right.$ $\left.g, \boldsymbol{g}_{\boldsymbol{i}^{n}}\right): \boldsymbol{Y} \rightarrow \boldsymbol{X}, n \in \mathbb{N}$, be defined in the same way by means of the pair $\left(g,\left(G_{i}^{k-1}\right)_{i \in \mathbb{N}}\right), k=i_{n+1}-i_{1}$.

Let us prove that $G_{n} F_{n} \simeq 1_{\boldsymbol{X} n}$ and $F_{n} G_{n} \simeq 1_{\boldsymbol{Y} n}, n \in \mathbb{N}$, by following the procedure used in the proof of Theorem 4.4. Let $n \in \mathbb{N}$ and $m \leq n$. For every $j_{1} \in \mathbb{N}$ and every $j_{1}^{\prime} \geq j_{1}$, put $j_{*}^{1}=g f\left(j_{1}^{\prime}\right)$, and let $j_{2}>j_{*}^{1} ; \ldots$; for every $j_{m}>j_{*}^{m-1}$ and every $j_{m}^{\prime} \geq j_{m}$, put $j_{*}^{m}=g f\left(j_{m}^{\prime}\right)$, and let $j_{m+1}>j_{*}^{m}$, $j_{m+2}>j_{m+1}, \ldots, j_{n+1}>j_{n}$.

Then condition $(H(n, m))$ for $F_{n} G_{n}$ and $1_{\boldsymbol{Y} n}$, i.e. the $m$-homotopy relation $\boldsymbol{f}_{\boldsymbol{j}^{n}} \boldsymbol{g}_{\boldsymbol{j}^{n}} \simeq_{m} \mathbf{1}_{\boldsymbol{Y} \boldsymbol{j}^{n}}$ holds by construction of the $n$-ladders. Therefore, 
$F_{n} G_{n} \simeq 1_{\boldsymbol{Y} n}$. Further, for every $i_{1} \in \mathbb{N}$ and every $i_{1}^{\prime} \geq i_{1}$, put $i_{*}^{1}=f g\left(i_{1}^{\prime}\right)$, and let $i_{2}>i_{*}^{1}, \ldots$; for every $i_{m}>i_{*}^{m-1}$ and every $i_{m}^{\prime} \geq i_{m}$, put $i_{*}^{m}=f g\left(i_{m}^{\prime}\right)$, and let $i_{m+1}>i_{*}^{m}, i_{m+2}>i_{m+1}, \ldots, i_{n+1}>i_{n}$. Then condition $(H(n, m))$ for $G_{n} F_{n}$ and $1_{\boldsymbol{X} n}$, i.e. the $m$-homotopy relation $\boldsymbol{g}_{\boldsymbol{i}^{n}} \boldsymbol{f}_{\boldsymbol{i}^{n}} \simeq_{m} \mathbf{1}_{\boldsymbol{X} \boldsymbol{i}^{n}}$ holds by construction of the $n$-ladders. Therefore, $G_{n} F_{n} \simeq 1_{\boldsymbol{X} n}$. Hence, there exist morphisms $\boldsymbol{F}=\left(\boldsymbol{F}_{n}\right) \in \underline{\mathcal{S}}^{*}(\mathbb{N})(\boldsymbol{X}, \boldsymbol{Y})$ and $\boldsymbol{G}=\left(\boldsymbol{G}_{n}\right) \in \underline{\mathcal{S}}^{*}(\mathbb{N})(\boldsymbol{Y}, \boldsymbol{X})$ such that $\boldsymbol{G} \boldsymbol{F}=\mathbf{1}_{\boldsymbol{X}}$ and $\boldsymbol{F} \boldsymbol{G}=\mathbf{1}_{\boldsymbol{Y}}$. Thus, $\boldsymbol{X} \cong \boldsymbol{Y}$ in $\underline{\mathcal{S}}^{*}(\mathbb{N})$.

Let us finally construct the appropriate functors relating our categories.

TheOREM 4.8. There exists a unique functor $\underline{T}^{*}: \underline{\mathcal{S}} h \rightarrow \underline{\mathcal{S}}^{*}(\mathbb{N})$, which keeps the objects fixed and is such that

$$
(\forall n \in \mathbb{N}) \underline{P}_{n}^{*} \underline{T}^{*}=\underline{T}_{n},
$$

where $\underline{T}_{n}: \underline{\mathcal{S}} h \rightarrow \underline{\mathcal{S}}(n)$ and $\underline{P}_{n}^{*}: \underline{\mathcal{S}}^{*}(\mathbb{N}) \rightarrow \underline{\mathcal{S}}(n)$ is the restriction of the $n$-projection functor $\underline{P}_{n}: \underline{\mathcal{S}}(\mathbb{N}) \rightarrow \underline{\mathcal{S}}(n)$ to the subcategory $\underline{\mathcal{S}}^{*}(\mathbb{N})$.

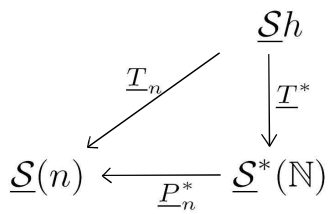

Consequently, there exists a unique functor $T^{*}: \mathcal{S} h \rightarrow \mathcal{S}^{*}(\mathbb{N})$, which keeps the objects fixed and is such that

$$
(\forall n \in \mathbb{N}) P_{n}^{*} T^{*}=T_{n},
$$

where $T_{n}: \mathcal{S} h \rightarrow \mathcal{S}_{n}$ and $P_{n}^{*}: \mathcal{S}^{*}(\mathbb{N}) \rightarrow \mathcal{S}(n)$ is the restriction of the $n$ projection functor $P_{n}: \mathcal{S}(\mathbb{N}) \rightarrow \mathcal{S}(n)$ to the subcategory $\mathcal{S}^{*}(\mathbb{N})$.

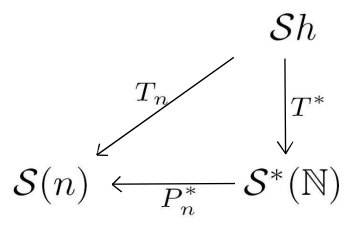

Proof. By the proof of Theorem 3.2, the image $\underline{T}[\underline{\mathcal{S}} h] \subseteq \underline{\mathcal{S}}^{*}(\mathbb{N})$. The conclusions follow by Theorem 3.2 and Corollary 3.5.

\section{The $q^{*}$-EQUivalence}

Here is another application of the "subshape theory". K. Borsuk had defined the relations of quasi-equivalence $\stackrel{q}{\simeq}$ and quasi-affinity $\stackrel{q}{\leftrightarrow}$ of compacta in terms of fundamental sequences between compacta lying in AR-spaces (see [2]). In order to characterize these relations in a category framework, the first 
named author adapted in [9] the original definitions in terms of the MardešićSegal shape theory [8]. Let us briefly sketch the indispensable definitions and facts from $[9]$.

Let $\boldsymbol{f}=\left(f,\left[f_{j}\right]\right), \boldsymbol{f}^{\prime}=\left(f^{\prime},\left[f_{j}^{\prime}\right]\right) \in \underline{\mathcal{S}}(\boldsymbol{X}, \boldsymbol{Y})$ and let $s \in \mathbb{N}$. Then $\boldsymbol{f}$ is said to be $s$-homotopic to $\boldsymbol{f}^{\prime}$, denoted by $\boldsymbol{f} \simeq{ }_{s} \boldsymbol{f}^{\prime}$, provided

$$
\left(\forall j \in[1, s]_{\mathbb{N}}\right)\left(\exists i=i(j) \geq f(j), f^{\prime}(j)\right)\left[f_{j}\right]\left[p_{f(j) i}\right]=\left[f_{j}^{\prime}\right]\left[p_{f^{\prime}(j) i}\right] .
$$

Observe that

$$
\boldsymbol{f} \simeq \boldsymbol{f}^{\prime} \Longleftrightarrow(\forall s \in \mathbb{N}) \boldsymbol{f} \simeq_{s} \boldsymbol{f}^{\prime}
$$

where $\simeq$ is the usual homotopy relation on $\underline{\mathcal{S}}$. Then,

(i) For every $s \in \mathbb{N}$, the relation $\simeq_{s}$ is an equivalence relation on each set $\underline{\mathcal{S}}(\boldsymbol{X}, \boldsymbol{Y})$

(ii) $\left(\forall s^{\prime} \leq s\right) \boldsymbol{f} \simeq{ }_{s} \boldsymbol{f}^{\prime} \Rightarrow \boldsymbol{f} \simeq{ }_{s^{\prime}} \boldsymbol{f}^{\prime}$.

Moreover, for every $s \in \mathbb{N}$, the relation $\simeq_{s}$ is natural from the right in the category $\underline{\mathcal{S}}$, i.e.

$$
(\forall \boldsymbol{h}: \boldsymbol{W} \rightarrow \boldsymbol{X}) \boldsymbol{f} \simeq_{s} \boldsymbol{f}^{\prime} \Rightarrow \boldsymbol{f h} \simeq_{s} \boldsymbol{f}^{\prime} \boldsymbol{h} .
$$

On the other side, if $\boldsymbol{g}: \boldsymbol{Y} \rightarrow \boldsymbol{Z}$ then

$$
\boldsymbol{f} \simeq_{s} \boldsymbol{f}^{\prime} \Rightarrow \boldsymbol{g f} \simeq_{s^{\prime}} \boldsymbol{g} \boldsymbol{f}^{\prime}
$$

whenever $g\left[\left[1, s^{\prime}\right]_{\mathbb{N}}\right] \subseteq[1, s]_{\mathbb{N}}$.

Let $\boldsymbol{X}$ and $\boldsymbol{Y}$ be compact ANR-sequences. Then $\boldsymbol{X}$ is said to be quasiequivalent to $\boldsymbol{Y}$, denoted by $\boldsymbol{X} \stackrel{q}{\simeq} \boldsymbol{Y}$, if for every $n \in \mathbb{N}$ there exist morphisms $\boldsymbol{f} \in \underline{\mathcal{S}}(\boldsymbol{X}, \boldsymbol{Y})$ and $\boldsymbol{g} \in \underline{\mathcal{S}}(\boldsymbol{Y}, \boldsymbol{X})$ such that $\boldsymbol{g} \boldsymbol{f} \simeq_{n} 1_{\boldsymbol{X}}$ and $\boldsymbol{f} \boldsymbol{g} \simeq_{n} 1_{\boldsymbol{Y}} . \boldsymbol{X}$ is said to be quasi-affinite to $\boldsymbol{Y}$, denoted by $\boldsymbol{X} \stackrel{q}{\leftrightarrow} \boldsymbol{Y}$, if for every $n \in \mathbb{N}$ there exist morphisms $\boldsymbol{f}, \boldsymbol{f}^{\prime} \in \underline{\mathcal{S}}(\boldsymbol{X}, \boldsymbol{Y})$ and $\boldsymbol{g}, \boldsymbol{g}^{\prime} \in \underline{\mathcal{S}}(\boldsymbol{Y}, \boldsymbol{X})$ such that $\boldsymbol{g} \boldsymbol{f} \simeq_{n} 1_{\boldsymbol{X}}$ and $\boldsymbol{f}^{\prime} \boldsymbol{g}^{\prime} \simeq_{n} 1_{\boldsymbol{Y}}$.

This relations $\stackrel{q}{\simeq}$ and $\stackrel{q}{\leftrightarrow}$ are shape invariant relations on the class $\mathrm{Ob} \underline{\mathcal{S}}$. By [9], Theorem 1, if $X, Y$ are compact metrizable spaces and if $\boldsymbol{X}, \boldsymbol{Y}$ are arbitrary with them associated compact ANR-sequences respectively, then

$$
X \stackrel{q}{\simeq} Y \Longleftrightarrow \boldsymbol{X} \stackrel{q}{\simeq} \boldsymbol{Y}
$$

and

$$
X \stackrel{q}{\leftrightarrow} Y \Longleftrightarrow X \stackrel{q}{\leftrightarrow} \boldsymbol{Y}
$$

Consequently, a compactum $X$ is quasi-equivalent to a compactum $Y, X \stackrel{q}{\simeq} Y$, if and only if, for every $n \in \mathbb{N}$, there exist morphisms $\boldsymbol{f}^{n}: \boldsymbol{X} \rightarrow \boldsymbol{Y}$ and $\boldsymbol{g}^{n}: \boldsymbol{Y} \rightarrow \boldsymbol{X}$ such that $\boldsymbol{g}^{n} \boldsymbol{f}^{n} \simeq_{n} 1_{\boldsymbol{X}}$ and $\boldsymbol{f}^{n} \boldsymbol{g}^{n} \simeq_{n} 1_{\boldsymbol{Y}}$, where $X=\lim \boldsymbol{X}$ and $Y=\boldsymbol{Y}$. Further, $X$ is quasi-affinite to $Y, X \stackrel{q}{\leftrightarrow} Y$, if and only if, for every $n \in \mathbb{N}$, there exist morphisms $\boldsymbol{f}^{n}, \boldsymbol{f}^{\prime n}: \boldsymbol{X} \rightarrow \boldsymbol{Y}$ and $\boldsymbol{g}^{n}, \boldsymbol{g}^{\prime n}: \boldsymbol{Y} \rightarrow \boldsymbol{X}$ such that $\boldsymbol{g}^{n} \boldsymbol{f}^{n} \simeq_{n} 1_{\boldsymbol{X}}$ and $\boldsymbol{f}^{\prime n} \boldsymbol{g}^{\prime n} \simeq_{n} 1_{\boldsymbol{Y}}$

One may assume, without loss of generality, that all the morphisms realizing the relations $\boldsymbol{X} \stackrel{q}{\simeq} \boldsymbol{Y}$ and $\boldsymbol{X} \stackrel{q}{\leftrightarrow} \boldsymbol{Y}$ are special morphisms. We may also 
assume that $n^{\prime} \geq n$ implies $f^{n^{\prime}} \geq f^{n}, g^{n^{\prime}} \geq g^{n}$ etc. Further, it is obvious that the conditions $\boldsymbol{g}^{n} \boldsymbol{f}^{n} \simeq_{n} 1_{\boldsymbol{X}}, \boldsymbol{f}^{n} \boldsymbol{g}^{n} \simeq_{n} 1_{\boldsymbol{Y}}$ etc. can be relaxed to condition $\boldsymbol{g}^{n} \boldsymbol{f}^{n} \simeq_{s_{n}} 1_{\boldsymbol{X}}, \boldsymbol{f}^{n} \boldsymbol{g}^{n} \simeq_{s_{n}} 1_{\boldsymbol{Y}}$ etc., where $\left(s_{n}\right)$ is an increasing unbounded sequence in $\mathbb{N} \cup\{0\}$.

In paper [9] the first named author constructed a certain category $\underline{\mathcal{K}}$ which describes the relations $\stackrel{q}{\simeq}$ and $\stackrel{q}{\leftrightarrow}$ by means of an appropriate relation on the morphisms of $\underline{\mathcal{K}}$. The objects of $\underline{\mathcal{K}}$ are all compact ANR-sequences, $\mathrm{Ob} \underline{\mathcal{K}}=\mathrm{Ob} \underline{\mathcal{S}}$, while

$$
\underline{\mathcal{K}}(\boldsymbol{X}, \boldsymbol{Y})=\left\{F=\left(\boldsymbol{f}^{n}\right) \mid \boldsymbol{f}^{n} \in \underline{\mathcal{S}}(\boldsymbol{X}, \boldsymbol{Y}) \text { special, } n \in \mathbb{N}\right\} .
$$

The composition in Mor $\underline{\mathcal{K}}$ is the coordinatewise composition, i.e.

$$
G F=\left(\boldsymbol{g}^{n} \boldsymbol{f}^{n}\right)=\left(\left(f^{n} g^{n},\left[g_{k}^{n} f_{g^{n}(k)}^{n}\right]\right)\right),
$$

while the identity morphism on an object $\boldsymbol{X} \in \mathrm{Ob} \underline{\mathcal{K}}$ is $1_{\boldsymbol{X}}=\left(1_{\boldsymbol{X}}^{n}\right)$, where $1_{\boldsymbol{X}}^{n}=1_{\boldsymbol{X}}$ for each $n \in \mathbb{N}$.

Further, a morphism $F=\left(\boldsymbol{f}^{n}\right) \in \underline{\mathcal{K}}(\boldsymbol{X}, \boldsymbol{Y})$ is said to be quasi-homotopic to a morphism $F^{\prime}=\left(\boldsymbol{f}^{\prime n}\right) \in \underline{\mathcal{K}}(\boldsymbol{X}, \boldsymbol{Y})$, denoted by $F \stackrel{q}{\simeq} F^{\prime}$, provided $\boldsymbol{f}^{n} \simeq_{n}$ $\boldsymbol{f}^{\prime n}$ for almost all $n$. The quasi-homotopy relation $\stackrel{q}{\simeq}$ is an equivalence relation on each set $\underline{\mathcal{K}}(\boldsymbol{X}, \boldsymbol{Y})$. It is also natural from the right, i.e.

$$
(\forall H \in \underline{\mathcal{K}}(\boldsymbol{W}, \boldsymbol{X}))\left(\forall F, F^{\prime} \in \underline{\mathcal{K}}(\boldsymbol{X}, \boldsymbol{Y})\right) F \stackrel{q}{\simeq} F^{\prime} \Rightarrow F H \stackrel{q}{\simeq} F^{\prime} H .
$$

Unfortunately, the quasi-homotopy relation $\stackrel{q}{\simeq}$ is not natural from the left in the category $\underline{\mathcal{K}}$, so there is no corresponding quotient category. Nevertheless, by [9], Theorem 3,

$\boldsymbol{X} \stackrel{q}{\simeq} \boldsymbol{Y}$ if and only if there exist morphisms $F \in \underline{\mathcal{K}}(\boldsymbol{X}, \boldsymbol{Y})$ and $G \in$ $\underline{\mathcal{K}}(\boldsymbol{Y}, \boldsymbol{X})$ such that $G F \stackrel{q}{\simeq} 1_{\boldsymbol{X}}$ and $F G \stackrel{q}{\simeq} 1_{\boldsymbol{Y}}$.

Similarly,

$\boldsymbol{X} \stackrel{q}{\leftrightarrow} \boldsymbol{Y}$ if and only if there exist morphisms $F, F^{\prime} \in \underline{\mathcal{K}}(\boldsymbol{X}, \boldsymbol{Y})$ and $G, G^{\prime} \in \underline{\mathcal{K}}(\boldsymbol{Y}, \boldsymbol{X})$ such that $G F \stackrel{q}{\simeq} 1_{\boldsymbol{X}}$ and $F^{\prime} G^{\prime} \stackrel{q}{\simeq} 1_{\boldsymbol{Y}}$.

In [9] it was also shown that for a slight strengthening of the Borsuk quasi-equivalence as well as the quasi-affinity, reinterpreted as above, there exists a category characterization. Let $\boldsymbol{X}, \boldsymbol{Y} \in \mathrm{Ob}(\underline{\mathcal{S}})$. Then $\boldsymbol{X}$ is said to be $\bar{q}$-equivalent to $\boldsymbol{Y}$, denoted by $\boldsymbol{X} \stackrel{\bar{q}}{\simeq} \boldsymbol{Y}$, provided $\boldsymbol{X} \stackrel{q}{\simeq} \boldsymbol{Y}$ and there exists a pair $F=\left(\boldsymbol{f}^{n}\right), G=\left(\boldsymbol{g}^{n}\right)$ of morpisms realizing this relation in the category $\underline{\mathcal{K}}$ such that

$$
(\forall i, j \in \mathbb{N})\left(f^{n}(j)\right),\left(g^{n}(i)\right) \text { are bounded sequences. }
$$

Further, $\boldsymbol{X}$ is said to be $\bar{q}$-affinite to $\boldsymbol{Y}$, denoted by $\boldsymbol{X} \stackrel{\bar{q}}{\leftrightarrow} \boldsymbol{Y}$, whenever $\boldsymbol{X} \stackrel{q}{\leftrightarrow} \boldsymbol{Y}$ and there exist morphisms $F=\left(\boldsymbol{f}^{n}\right), F^{\prime}=\left(\boldsymbol{f}^{\prime n}\right), G=\left(\boldsymbol{g}^{n}\right)$ and $G^{\prime}=\left(\boldsymbol{g}^{\prime n}\right)$ which realize this relation in the category $\underline{\mathcal{K}}$, such that

$$
(\forall i, j \in \mathbb{N})\left(f^{n}(j)\right),\left(f^{\prime n}(j)\right),\left(g^{n}(i)\right),\left(g^{\prime n}(i)\right) \text { are bounded sequences. }
$$


For a pair $X, Y$ of compacta, we define $X \stackrel{\bar{q}}{\simeq} Y(X \stackrel{\bar{q}}{\leftrightarrow} Y)$ if $\boldsymbol{X} \stackrel{\bar{q}}{\simeq} \boldsymbol{Y}$ $(\boldsymbol{X} \stackrel{\bar{q}}{\leftrightarrow} \boldsymbol{Y})$ for some (equivalently, for any) pair $\boldsymbol{X}, \boldsymbol{Y}$ of the associated compact ANR-sequences.

Let $\overline{\mathcal{K}}$ be the subcategory of $\underline{\mathcal{K}}$ consisting of $\mathrm{Ob} \overline{\mathcal{K}}=\mathrm{Ob} \underline{\mathcal{K}}=\mathrm{Ob}(\underline{\mathcal{S}})$ and Mor $\overline{\mathcal{K}} \subseteq$ Mor $\underline{\mathcal{K}}$ such that each $\overline{\mathcal{K}}(\boldsymbol{X}, \boldsymbol{Y}) \subseteq \underline{\mathcal{K}}(\boldsymbol{X}, \boldsymbol{Y})$ consists of all the morphisms $F=\left(\boldsymbol{f}^{n}\right)$, where all $\boldsymbol{f}^{n}=\left(f^{n},\left[f_{j}^{n}\right]\right)$ have a unique index function $f=f^{n}, n \in \mathbb{N}$. Such a morphism is denoted by $F=\left(f, \boldsymbol{f}^{n}\right)$. A morphism $F=\left(f, \boldsymbol{f}^{n}\right): \boldsymbol{X} \rightarrow \boldsymbol{Y}$ is said to be $\bar{q}$-homotopic to an $F^{\prime}=$ $\left(f^{\prime}, \boldsymbol{f}^{\prime n}\right): \boldsymbol{X} \rightarrow \boldsymbol{Y}$, denoted by $F \stackrel{\bar{q}}{\simeq} F^{\prime}$, provided there exists an increasing and unbounded sequence $\left(s_{n}\right)$ in $\mathbb{N}_{0}$ such that $\boldsymbol{f}^{n} \simeq s_{n} \boldsymbol{f}^{\prime n}$, whenever $s_{n} \geq 1$. The key fact is that the $\bar{q}$-homotopy relation $\stackrel{\bar{q}}{\simeq}$ is a natural equivalence relation on Mor $\overline{\mathcal{K}}$. Therefore, there exists the corresponding quotient category $\overline{\mathcal{K}} / \underset{\sim}{\sim} \equiv$ $\overline{\mathcal{Q}}$. Observe that the quotient category $\overline{\mathcal{Q}}$ yields the associated category $\overline{\mathcal{Q}}$ on compacta such that

$$
O b \mathcal{Q}=O b(c \mathcal{M})
$$

and

$$
\mathcal{Q}(X, Y) \approx \overline{\mathcal{Q}}(\boldsymbol{X}, \boldsymbol{Y})
$$

where $\boldsymbol{X}, \boldsymbol{Y}$ are any compact ANR-sequences associated with $X, Y$ respectively. (For a given pair $X, Y$, any set $\overline{\mathcal{Q}}(\boldsymbol{X}, \boldsymbol{Y})$ may represent $\mathcal{Q}(X, Y)$.) An important result is the following one ([9], Theorem 6$)$ :

For every pair $\boldsymbol{X}, \boldsymbol{Y} \in \mathrm{Ob}(\underline{\mathcal{S}})$,

$$
\begin{aligned}
& \boldsymbol{X} \stackrel{\bar{q}}{\simeq} \boldsymbol{Y} \text { if and only if } \boldsymbol{X} \cong \boldsymbol{Y} \text { in } \overline{\mathcal{Q}} ; \\
& \boldsymbol{X} \stackrel{\bar{q}}{\leftrightarrow} \boldsymbol{Y} \quad \text { if and only if } \boldsymbol{X} \leq \boldsymbol{Y} \text { and } \boldsymbol{Y} \leq \boldsymbol{X} \text { in } \overline{\mathcal{Q}} ;
\end{aligned}
$$

Consequently, for every pair $X, Y$ of compacta, $X \stackrel{\bar{q}}{\simeq} Y(X \stackrel{\bar{q}}{\leftrightarrow} Y)$ if and only if $X \cong Y(X \leq Y \wedge Y \leq X)$ in $\mathcal{Q}$.

Moreover [9], Theorem 7), there exist functors $Q: H c \mathcal{M} \rightarrow \mathcal{Q}$ and $\Gamma$ : $\mathcal{S} h \rightarrow \mathcal{Q}$, which keep the objects fixed and $\Gamma S=Q$, where $S: H c \mathcal{M} \rightarrow \mathcal{S} h$ is the ordinary shape functor.

According to [9], Remark 8. (b), the $\bar{q}$-equivalence admits a slight strengthening in the following way: A morphism $F=\left(f, \boldsymbol{f}^{n}\right) \in \overline{\mathcal{K}}(\boldsymbol{X}, \boldsymbol{Y})$ is said to be uniformly $\bar{q}$-homotopic to a morphism $F^{\prime}=\left(f^{\prime}, \boldsymbol{f}^{\prime n}\right) \in \overline{\mathcal{K}}(\boldsymbol{X}, \boldsymbol{Y})$, denoted by $F \stackrel{q^{*}}{\simeq} F^{\prime}$, provided $F \stackrel{\bar{q}}{\simeq} F^{\prime}$ and there exists a sequence $\left(i_{j}\right)$ in $\mathbb{N}$, $i_{j} \geq f(j), f^{\prime}(j)$, such that

$$
(\forall n \in \mathbb{N})\left(\forall j \in\left[1, s_{n}\right]_{\mathbb{N}}\right)\left[f_{j}^{n}\right]\left[p_{f(j) i_{j}}\right]=\left[f_{j}^{\prime n}\right]\left[p_{f^{\prime}(j) i_{j}}\right]
$$

where $\left(s_{n}\right)$ is a realizing sequence for $F \stackrel{\bar{q}}{\simeq} F^{\prime}$. One easily checks that $\stackrel{q^{*}}{\simeq}$ is a natural equivalence relation on $\overline{\mathcal{K}}$. Thus, there exist the corresponding quotient category $\overline{\mathcal{K}} / \underset{q^{*}}{\simeq} \equiv \overline{\mathcal{Q}}^{*}$ and the associated category $\mathcal{Q}^{*}$ on compacta. 
Further, there exist functors $Q^{*}: H c \mathcal{M} \rightarrow \mathcal{Q}^{*}$ and $\Gamma^{*}: \mathcal{S} h \rightarrow \mathcal{Q}^{*}$, which keep the objects fixed and $\Gamma^{*} S=Q^{*}$. Moreover, there exists a functor $\Pi: \mathcal{Q}^{*} \rightarrow \mathcal{Q}$ such that $Q=\Gamma Q^{*}$ and $\Gamma=\Pi \Gamma^{*}$.

Let $\boldsymbol{X}, \boldsymbol{Y}$ be a pair of compact ANR-sequences. Then, $\boldsymbol{X}$ is said to be $q^{*}$-equivalent to $\boldsymbol{Y}$, denoted by $\boldsymbol{X} \stackrel{q^{*}}{\simeq} \boldsymbol{Y}$, provided there exists a pair of morphisms $F: \boldsymbol{X} \rightarrow \boldsymbol{Y}, G: \boldsymbol{Y} \rightarrow \boldsymbol{X}$ in $\overline{\mathcal{K}}$ such that $G F \stackrel{q^{*}}{\simeq} 1_{\boldsymbol{X}}$ and $F G \stackrel{q^{*}}{\simeq} 1_{\boldsymbol{Y}}$. Clearly, this means $\boldsymbol{X} \cong \boldsymbol{Y}$ in $\overline{\mathcal{Q}}^{*}$. The $q^{*}$-affinity, $\boldsymbol{X} \stackrel{q^{*}}{\leftrightarrow} \boldsymbol{Y}$, means $\boldsymbol{X} \leq \boldsymbol{Y}$ and $\boldsymbol{Y} \leq \boldsymbol{X}$ in $\overline{\mathcal{Q}}^{*}$. The $q^{*}$-equivalence ( $q^{*}$-affinity) of compacta is the induced relation in the category $\mathcal{Q}^{*}$.

In order to relate the above mentioned relations with a certain subshape category, let us recall our category $\underline{\mathcal{L}}(1)$. Let $\underline{\mathcal{A}}(1)$ be the collection consisting of $\mathrm{Ob} \underline{\mathcal{A}}(1)=\mathrm{Ob} \underline{\mathcal{L}}(1)=\mathrm{Ob} \underline{\mathcal{S}}$ and of the morphism sets

$$
\underline{\mathcal{A}}(1)(\boldsymbol{X}, \boldsymbol{Y}) \subseteq \underline{\mathcal{L}}(1)(\boldsymbol{X}, \boldsymbol{Y})=\underline{L}_{1}(\boldsymbol{X}, \boldsymbol{Y}),
$$

such that a 1-hyperladder $F_{1}=\left(\boldsymbol{f}_{\boldsymbol{j}^{1}}\right): \boldsymbol{X} \rightarrow \boldsymbol{Y}$ belongs to $\underline{\mathcal{A}}(1)(\boldsymbol{X}, \boldsymbol{Y})$, whenever each of its 1-ladder $\boldsymbol{f}_{\boldsymbol{j}^{1}}, \boldsymbol{j}^{1} \in \boldsymbol{J}(1)$, is induced by a special morphism $\boldsymbol{f}: \boldsymbol{X} \rightarrow \boldsymbol{Y}$ of $\underline{\mathcal{S}}$. Clearly, the identity 1-hyperladder $1_{\boldsymbol{X} 1}=\left(\mathbf{1}_{\boldsymbol{X} \boldsymbol{i}^{1}}\right)$ belongs to $\underline{\mathcal{A}}(1)(\boldsymbol{X}, \boldsymbol{X})$. Further, if $F_{1}=\left(\boldsymbol{f}_{\boldsymbol{j}^{1}}\right) \in \underline{\mathcal{A}}(1)(\boldsymbol{X}, \boldsymbol{Y})$ and $G_{1}=$ $\left(\boldsymbol{g}_{\boldsymbol{k}^{1}}\right) \in \underline{\mathcal{A}}(1)(\boldsymbol{Y}, \boldsymbol{Z})$, then $G_{1} F_{1}=\left(\boldsymbol{g}_{\boldsymbol{k}^{1}} \boldsymbol{f}_{\boldsymbol{k}^{1}}\right) \in \underline{\mathcal{A}}(1)(\boldsymbol{X}, \boldsymbol{Z})$. Namely, if $\boldsymbol{f}_{\boldsymbol{k}^{1}}$ is induced by an $\boldsymbol{f}$, and $\boldsymbol{g}_{\boldsymbol{k}^{1}}$ is induced by a $\boldsymbol{g}$, then $\boldsymbol{g}_{\boldsymbol{k}^{1}} \boldsymbol{f}_{\boldsymbol{k}^{1}}$ is induced by $\boldsymbol{g} \boldsymbol{f}$. Therefore, $\underline{\mathcal{A}}(1)$ is a subcategory of $\underline{\mathcal{L}}(1)$. Let $\underline{\mathcal{A}}_{1}(1) \subseteq \underline{\mathcal{A}}(1)$ be the subcategory determined by the following two conditions

(i) there exists a unique index function $f$ for all 1-ladders of a 1hyperladder $F_{1}=\left(f, \boldsymbol{f}_{\boldsymbol{j}^{1}}\right) \in \underline{\mathcal{A}}_{1}(1)(\boldsymbol{X}, \boldsymbol{Y})$;

(ii) for every 1-ladder $\boldsymbol{f}_{\boldsymbol{j}^{1}}$ of a 1-hyperladder $F_{1}=\left(f, \boldsymbol{f}_{\boldsymbol{j}^{1}}\right) \in \underline{\mathcal{A}}_{1}(1)(\boldsymbol{X}, \boldsymbol{Y})$, the starting index $j_{1}=1$, i.e. $\boldsymbol{j}^{1}=\left(1, j_{2}\right)$;

Let $\underline{\mathcal{B}}_{1}(1)$ be the corresponding quotient category $\underline{\mathcal{A}}_{1}(1) / \simeq$. Finally, let $\underline{\mathcal{B}}^{*} \subseteq \underline{\mathcal{S}}(1)$ be the maximal subcategory such that every morphism $\boldsymbol{F}_{1}=$ $\left[F_{1}\right] \in \underline{\mathcal{B}}^{*}(\boldsymbol{X}, \boldsymbol{Y})$ admits a representative $F_{1}=\left(f, \boldsymbol{f}_{\boldsymbol{j}^{1}}\right) \in \underline{\mathcal{A}}_{1}(1)(\boldsymbol{X}, \boldsymbol{Y})$. The corresponding category of compacta is denoted by $\mathcal{B}^{*}$.

Notice that the functor $\underline{T}_{1}: \underline{\mathcal{S}} h \rightarrow \underline{\mathcal{S}}(1)$ yields the functor $\underline{T}_{1,1}: \underline{\mathcal{S}} h \rightarrow$ $\underline{\mathcal{S}}_{1}(1)\left(j_{1}=1\right)$. Furthermore, by the proof of Theorem 3.2, $\underline{T}_{1,1}[\underline{\mathcal{S}} h] \subseteq \underline{\mathcal{B}}^{*}$. Thus, $\underline{T}_{1}$ yields a functor $\underline{T}^{*}: \underline{\mathcal{S}} h \rightarrow \underline{\mathcal{B}}^{*}$. The corresponding functor on compacta is denoted by $B^{*}: \mathcal{S} h \rightarrow \mathcal{B}^{*}$.

Theorem 5.1. There exists a functor $\underline{A}^{*}: \underline{\mathcal{B}}^{*} \rightarrow \overline{\mathcal{Q}}^{*}$, which keeps the objects fixed and is such that $\underline{A}^{*} \underline{T}^{*}=\bar{\Gamma}^{*}$. Consequently, there exists a functor $A^{*}: \mathcal{B}^{*} \rightarrow \mathcal{Q}^{*}$, which keeps the objects fixed and is such that $A^{*} T^{*}=\Gamma^{*}$.

Proof. The category $\underline{\mathcal{B}}^{*}$ and the functor $\underline{T}^{*}$ are already constructed. For every $\boldsymbol{X} \in \mathrm{Ob}\left(\underline{\mathcal{B}}^{*}\right)=\mathrm{Ob}(\underline{\mathcal{S}})$, put $\underline{A}^{*}(\boldsymbol{X})=\overline{\boldsymbol{X}}$. Let $\boldsymbol{F}_{1} \in \underline{\mathcal{B}}^{*}(\boldsymbol{X}, \boldsymbol{Y})$. Then there exists a representative $F_{1}=\left(f, \boldsymbol{f}_{\boldsymbol{j}^{1}}\right)$ of $\boldsymbol{F}_{1}=\left[F_{1}\right]$, such that $j_{1}=1, f$ is 
the index function for all $\boldsymbol{f}_{\boldsymbol{j}^{1}}$ and every $\boldsymbol{f}_{\boldsymbol{j}^{1}}$ is induced by a special morphism of inverse sequences $\boldsymbol{f}\left(\boldsymbol{j}^{1}\right): \boldsymbol{X} \rightarrow \boldsymbol{Y}$. Recall that $\boldsymbol{j}^{1}=\left(1, j_{2}\right) \in \boldsymbol{J}(1)$. By conditions $\left(S(1,1)_{1,2}\right)$ with $j_{1}=1$, for every $n=j_{1}^{\prime} \in \mathbb{N}$, there exists an $i^{1}(n) \geq f(n)$ such that, for every $j_{2}>i^{1}$, the 1-ladder $\boldsymbol{f}_{\boldsymbol{j}^{1}}$ (i.e. the morphism $\left.\boldsymbol{f}\left(\boldsymbol{j}^{1}\right): \boldsymbol{X} \rightarrow \boldsymbol{Y}\right)$ satisfies

$$
\left(\forall j \in[1, n]_{\mathbb{N}}\right) f(j) \leq f(n) \leq i^{1} .
$$

Let $\left(j_{2, n}\right)$ be a strictly increasing sequence in $\mathbb{N}$ such that $i^{1}(n)<j_{2, n}$, for every $n$. Put

$$
\boldsymbol{f}^{n}=\boldsymbol{f}\left(\boldsymbol{j}_{n}^{1}\right), \boldsymbol{j}_{n}^{1}=\left(1, j_{2, n}\right), n \in \mathbb{N} .
$$

Then $F=\left(f, \boldsymbol{f}^{n}\right) \in \overline{\mathcal{K}}(\boldsymbol{X}, \boldsymbol{Y})$. If $F^{\prime}=\left(f^{\prime}, \boldsymbol{f}^{\prime n}\right) \in \overline{\mathcal{K}}(\boldsymbol{X}, \boldsymbol{Y})$ is defined in the same way (via the same $\left(j_{2, n}\right)$ ), by using another representative $F_{1}^{\prime}=\left(f^{\prime}, \boldsymbol{f}_{\boldsymbol{j}^{1}}^{\prime}\right) \in \boldsymbol{F}_{1}$, then condition $(H(1,1))$ implies $F \stackrel{q}{\simeq} F^{\prime}$ as well as the appropriate uniform condition, i.e. $F \stackrel{q^{*}}{\simeq} F^{\prime}$. Thus, the function

$$
\boldsymbol{F}_{1} \mapsto \underline{A}^{*}\left(\boldsymbol{F}_{1}\right)=\boldsymbol{F}^{*}=[F]_{q^{*}}
$$

is well defined. Moreover, $\underline{A}^{*}\left(\mathbf{1}_{\boldsymbol{X} 1}\right)=\mathbf{1}_{\boldsymbol{X}}^{*}$ obviously holds. Notice that, if $F$ is defined by an $F_{1}, G$ is defined by a $G_{1}$ and $U$ is defined by $G_{1} F_{1}$ (via the same sequences, i.e. the same sequence of indices $j_{n}^{1}$ in $\underline{J}(1)$ with $j_{1}=1$ ), then $U=G F$. Therefore,

$$
\begin{aligned}
\underline{A}^{*}\left(\boldsymbol{G}_{1} \boldsymbol{F}_{1}\right) & =\underline{A}^{*}\left(\left[G_{1}\right]\left[F_{1}\right]\right)=\underline{A}^{*}\left(\left[G_{1} F_{1}\right]\right)=\boldsymbol{U}^{*} \\
& =[U]_{\underline{q^{*}}}=[G F]_{\underline{q^{*}}}=[G]_{\underline{q^{*}}}[F]_{\underline{q^{*}}}=\boldsymbol{G}^{*} \boldsymbol{F}^{*}=\underline{A}^{*}\left(\boldsymbol{G}_{1}\right) \underline{A}^{*}\left(\boldsymbol{F}_{1}\right) .
\end{aligned}
$$

Hence, $\underline{A}^{*}: \underline{\mathcal{B}}^{*} \rightarrow \overline{\mathcal{Q}}^{*}$ is a functor (which is not unique!). The rest of the proof is trivial (see also Remark 8.(b) of [9]).

Corollary 5.2. $\boldsymbol{X} \cong \boldsymbol{Y}$ in $\underline{\mathcal{B}}^{*}$ implies $\boldsymbol{X} \stackrel{q^{*}}{\simeq} \boldsymbol{Y}, \boldsymbol{X} \stackrel{\bar{q}}{\simeq} \boldsymbol{Y}$ and $\boldsymbol{X} \stackrel{q}{\simeq} \boldsymbol{Y}$. Consequently, $X \cong Y$ in $\mathcal{B}^{*}$ implies $X \stackrel{q^{*}}{\simeq} Y, X \stackrel{\bar{q}}{\simeq} Y$ and $X \stackrel{q}{\simeq} Y$.

Concerning the converse, we have exhibited the following theorem:

TheOREm 5.3. There exists a functor $\underline{L}^{*}: \overline{\mathcal{Q}}^{*} \rightarrow \underline{\mathcal{B}}^{*}$, which keeps the objects fixed and is such that $\underline{A}^{*} \underline{L}^{*}=1 \overline{\mathcal{Q}}^{*}$ for every $\underline{A}^{*}$, and $\underline{L}^{*} \bar{\Gamma}^{*}=\underline{B}^{*}$, where $\bar{\Gamma}^{*}: \underline{\mathcal{S}} h \rightarrow \overline{\mathcal{Q}}^{*}$. Consequently, there exists a functor $L^{*}: \mathcal{Q}^{*} \rightarrow \mathcal{B}^{*}$, which keeps the objects fixed and is such that $A^{*} L^{*}=1_{\mathcal{Q}^{*}}$ for every $A^{*}$, and $L^{*} \Gamma^{*}=B^{*}$.

Proof. For every $\boldsymbol{X} \in O b \overline{\mathcal{Q}}^{*}$, put $\underline{L}^{*}(\boldsymbol{X})=\boldsymbol{X}$. Let $\boldsymbol{F}^{*} \in \overline{\mathcal{Q}}^{*}(\boldsymbol{X}, \boldsymbol{Y})$. Then $\boldsymbol{F}^{*}=[F]_{q^{*}}$, where $F=\left(f, \boldsymbol{f}^{n}\right) \in \overline{\bar{K}}(\boldsymbol{X}, \boldsymbol{Y})$. For every $\boldsymbol{j}^{1}=\left(1, j_{2}\right) \in$ $\boldsymbol{J}(1)$, let $\boldsymbol{f}_{\boldsymbol{j}^{1}}$ be the 1-ladder induced by the morphism $\boldsymbol{f}^{j_{2}-1}$. Let $F_{1}=$ $\left(f, \boldsymbol{f}_{\boldsymbol{j}^{1}}\right)$ be indexed by all $\boldsymbol{j}^{1}=\left(1, j_{2}\right) \in \boldsymbol{J}(1)$. Since $f$ is the index function for all 1-ladders $\boldsymbol{f}_{\boldsymbol{j}^{1}}$, conditions $\left(S(1,1)_{1,2}\right)$ with $j_{1}=1$ for $F_{1}$ hold. (Given 
any $j_{1}^{\prime} \geq 1$, put $i^{1}=f\left(j_{1}^{\prime}\right)$.) Thus, $F_{1} \in \underline{\mathcal{A}}_{1}(1)(\boldsymbol{X}, \boldsymbol{Y})$. If $F_{1}^{\prime}$ is defined in the same way by means of another representative $F^{\prime}=\left(f^{\prime}, \boldsymbol{f}^{\prime n}\right) \in \boldsymbol{F}^{*}$, then the uniformity in $F \stackrel{q^{*}}{\simeq} F^{\prime}$ implies condition $(H(1,1))$ with $j_{1}=1$ for $F_{1}$ and $F_{1}^{\prime}$, i.e. $F_{1} \simeq F_{1}^{\prime}$. Hence, the function $\boldsymbol{F}^{*} \mapsto \underline{L}^{*}\left(\boldsymbol{F}^{*}\right)=\boldsymbol{F}_{1}$ is well defined. Further, $\underline{L}^{*}\left(\mathbf{1}_{\boldsymbol{X}}^{*}\right)=\mathbf{1}_{\boldsymbol{X} 1}$. Notice that, if $F_{1}$ is defined by an $F, G_{1}$ is defined by a $G$ and $U_{1}$ is defined by $G F$, then $U_{1}=G_{1} F_{1}$. Therefore,

$$
\begin{aligned}
& \underline{L}^{*}\left(\boldsymbol{G}^{*} \boldsymbol{F}^{*}\right)=\underline{L}^{*}\left([G]_{\underline{q^{*}}}[F]_{\underline{q^{*}}}\right)=\underline{L}^{*}\left([G F]_{\underline{q^{*}}}\right)=\boldsymbol{U}_{1}=\left[U_{1}\right] \\
& =\left[G_{1} F_{1}\right]=\left[G_{1}\right]\left[F_{1}\right]=\boldsymbol{G}_{1} \boldsymbol{F}_{1}=\underline{L}^{*}\left(\boldsymbol{G}^{*}\right) \underline{L}^{*}\left(\boldsymbol{F}^{*}\right) .
\end{aligned}
$$

Hence, $\underline{L}^{*}: \overline{\mathcal{Q}}^{*} \rightarrow \underline{\mathcal{B}}^{*}$ is a functor. To verify that $\underline{A}^{*} \underline{L}^{*}=1 \overline{\mathcal{Q}}^{*}$, it suffices to observe that our constructions $F \mapsto F_{1}$ and $F_{1} \mapsto F$ (the proof of Theorem 5.1) yield $F \mapsto F^{\prime}$, where $F^{\prime}$ is a subsequence of $F$. The rest of the proof is trivial (see also Remark 8.(b) of [9]).

REMARK 5.4. The uniformity in $F \stackrel{q^{*}}{\simeq} F^{\prime}$ has been the essential assumption for the construction of the functor $\underline{L}^{*}: \overline{\mathcal{Q}}^{*} \rightarrow \underline{\mathcal{B}}^{*}$. We do not know whether there exists a similar functor from $\overline{\mathcal{Q}}$ to $\underline{\mathcal{B}}^{*}$.

Corollary 5.5. $\boldsymbol{X} \stackrel{q^{*}}{\simeq} \boldsymbol{Y}\left(\boldsymbol{X} \stackrel{q^{*}}{\leftrightarrow} \boldsymbol{Y}\right)$ if and only if $\boldsymbol{X} \cong \boldsymbol{Y}(\boldsymbol{X} \leq \boldsymbol{Y}$ and $\boldsymbol{Y} \leq \boldsymbol{X})$ in $\underline{\mathcal{B}}^{*}$. Consequently, $X \stackrel{q^{*}}{\simeq} Y\left(X \stackrel{q^{*}}{\leftrightarrow} Y\right)$ if and only if $X \cong Y$ $(X \leq Y$ and $Y \leq X)$ in $\mathcal{B}^{*}$.

The next theorem relates the $q^{*}$-equivalence and $S^{*}$-equivalence.

THEOREM 5.6. There exists a functor $\underline{B}^{*}: \underline{\mathcal{B}}^{*} \rightarrow \underline{\mathcal{S}}^{*}(\mathbb{N})$, which keeps the objects fixed. Consequently, there exists a functor $B^{*}: \mathcal{B}^{*} \rightarrow \mathcal{S}^{*}(\mathbb{N})$, which keps the object fixed.

Proof. It suffices to prove that there exists a functor $\underline{M}^{*}: \overline{\mathcal{Q}}^{*} \rightarrow \underline{\mathcal{S}}^{*}(\mathbb{N})$ which keeps the objects fixed. Then the desired functor $\underline{B}^{*}$ can be the composition $\underline{M}^{*} \underline{A}^{*}$ (see Theorem 5.1). For every object $\boldsymbol{X}$, put $\underline{M}^{*}(\boldsymbol{X})=\boldsymbol{X}$. Let $\boldsymbol{F}^{*} \in \overline{\mathcal{Q}}^{*}(\bar{X}, \boldsymbol{Y})$. Then $\boldsymbol{F}^{*}=[F]_{q^{*}}$, where $F=\left(f, \boldsymbol{f}^{n}\right) \in \overline{\mathcal{K}}(\boldsymbol{X}, \boldsymbol{Y})$. For every $n \in \mathbb{N}$ and every $\boldsymbol{j}^{n} \in \boldsymbol{J}(n)$, let the $n$-ladder $\boldsymbol{f}_{\boldsymbol{j}^{n}}$ be induced by the morphism $\boldsymbol{f}^{j_{n+1}-1}$. Let $F_{n}=\left(f, \boldsymbol{f}_{\boldsymbol{j}^{n}}\right)$ be indexed by all $\boldsymbol{j}^{n} \in \boldsymbol{J}(n)$. Since $f$ is the index function for all the $n$-ladders $\boldsymbol{f}_{-}^{j_{n}}$, conditions $\left(S(n, m)_{1,2}\right)$ for $F^{n}$ hold immediately. (Given any $j_{\lambda}^{\prime} \geq j_{\lambda}$, put $i^{\lambda}=f\left(j_{\lambda}^{\prime}\right)$.) Thus, $F_{n} \in \underline{\mathcal{L}}_{0}(n)(\boldsymbol{X}, \boldsymbol{Y})$. If $F_{n}^{\prime}=\left(f^{\prime}, \boldsymbol{f}^{\prime} \underline{-}_{n}\right)$ is defined in the same way by using another representative $F^{\prime}=\left(f^{\prime}, \boldsymbol{f}^{\prime n}\right) \in \boldsymbol{F}^{*}$, then the uniformity in $F \stackrel{q^{*}}{\simeq} F^{\prime}$ implies condition $(H(n, m)), m \leq n$, for $F_{n}$ and $F_{n}^{\prime}$, i.e. $F_{n} \simeq F_{n}^{\prime}$. (Given any $j_{\lambda}^{\prime} \geq j_{\lambda}$, put $i_{*}^{\lambda}=i_{j_{\lambda}^{\prime}}$ coming from $F \stackrel{q^{*}}{\simeq} F^{\prime}$.) Hence, for every $n \in \mathbb{N}$, the function $\boldsymbol{F}^{*} \mapsto \boldsymbol{F}_{n}$ is well defined. Therefore, the function $\boldsymbol{F}^{*} \mapsto \underline{M}^{*}\left(\boldsymbol{F}^{*}\right)=\left(\boldsymbol{F}_{n}\right)=\boldsymbol{F}$ is well defined. Moreover, since $\left(\boldsymbol{F}_{n}\right)$ has the constructed representative 
$\left(F_{n}\right), F_{n}=\left(f_{n}, \boldsymbol{f}_{\boldsymbol{j}^{n}}\right) \in \underline{\mathcal{L}}_{0}(n)(\boldsymbol{X}, \boldsymbol{Y})$, having the unique index function $f=f_{n}$ for every $n \in \mathbb{N}$, we infer that $\boldsymbol{F}=\left(\boldsymbol{F}_{n}\right) \in \underline{\mathcal{S}}^{*}(\mathbb{N})(\boldsymbol{X}, \boldsymbol{Y})$. Further, $\underline{M}^{*}\left(\mathbf{1}_{\boldsymbol{X}}^{*}\right)=\mathbf{1}_{\boldsymbol{X}}$. Notice that, for every $n \in \mathbb{N}$, if $F_{n}$ is defined by an $F$, $G_{n}$ is defined by a $G$ and $U_{n}$ is defined by $G F$, then $U_{n}=G_{n} F_{n}$. Therefore,

$$
\begin{aligned}
\underline{M}^{*}\left(\boldsymbol{G}^{*} \boldsymbol{F}^{*}\right) & =\underline{M}^{*}\left([G]_{q^{*}}[F]_{q^{*}}\right)=\underline{M}^{*}\left([G F]_{q^{*}}\right)=\boldsymbol{U} \\
& =\left(\boldsymbol{U}_{n}\right)=\left(\left[U_{n}\right]\right)=\left(\left[G_{n} F_{n}\right]\right)=\left(\left[G_{n}\right]\left[F_{n}\right]\right)=\left(\left[G_{n}\right]\right)\left(\left[F_{n}\right]\right) \\
& =\left(\boldsymbol{G}_{n}\right)\left(\boldsymbol{F}_{n}\right)=\boldsymbol{G F}=\underline{M}^{*}\left(\boldsymbol{G}^{*}\right) \underline{M}^{*}\left(\boldsymbol{F}^{*}\right) .
\end{aligned}
$$

Hence, $\underline{M}^{*}: \overline{\mathcal{Q}}^{*} \rightarrow \underline{\mathcal{S}}^{*}(\mathbb{N})$ is a functor.

Corollary 5.7. $\boldsymbol{X} \stackrel{q^{*}}{\simeq} \boldsymbol{Y}$ implies $S^{*}(\boldsymbol{X})=S^{*}(\boldsymbol{Y})$, but not conversely.

Consequently, $X \stackrel{q^{*}}{\simeq} Y$ implies $S^{*}(X)=S^{*}(Y)$, but not conversely.

Proof. By Corollary 5.5, $\boldsymbol{X} \stackrel{q^{*}}{\simeq} \boldsymbol{Y}$ if and only if $\boldsymbol{X} \cong \boldsymbol{Y}$ in $\underline{\mathcal{B}}^{*}$. By Theorem 4.7, for $\boldsymbol{X}, \boldsymbol{Y} \in O b \underline{\mathcal{S}}, S^{*}(\boldsymbol{X})=S^{*}(\boldsymbol{Y})$ if and only if $\overline{\boldsymbol{X}} \cong \boldsymbol{Y}$ in $\underline{\mathcal{S}}^{*}(\mathbb{N})$. The equality $S^{*}(\boldsymbol{X})=S^{*}(\boldsymbol{Y})$ follows by Theorem 5.6. Further, by [5], there exists a pair $X, Y$ of continua such that $S(X)=S(Y)$ and $S h(X) \neq S h(Y)$. By [7], Theorem $6, S^{*}(X)=S^{*}(Y)$ also holds. By [9], Example 5 (see also [7], Theorem 8), $X$ and $Y$ are not quasi-equivalent, and therefore, they are not $q^{*}$-equivalent.

\section{The Shape of FANR's}

First of all, let us describe a morphism $\Phi_{n} \in \mathcal{S}(n)(X, Y), n \in \mathbb{N} \cup\{\omega\}$, and a morphism $\Phi \in \mathcal{S}(\mathbb{N})(X, Y)$, where $X$ is a compactum and $Y$ is a compact ANR (or, more general, a compactum having the homotopy type of a compact ANR). Let $\boldsymbol{X}$ be a compact ANR inverse sequence associated with $X$, i.e. $X=\lim \boldsymbol{X}$, and let $\boldsymbol{Y}$ be the trivial (compact ANR) inverse sequence associated with $Y$, i.e. $Y_{j}=Y$, for every $j \in \mathbb{N}$ and $\left[q_{j j^{\prime}}\right]=\left[1_{Y}\right]$, for all $j \leq j^{\prime}$. Let $\boldsymbol{f}_{\boldsymbol{j}^{n}}: \boldsymbol{X} \rightarrow \boldsymbol{Y}$ be an $n$-ladder. Since $Y_{j}=Y$, for every $j, \boldsymbol{f}_{\boldsymbol{j}^{n}}$ determines a (special) morphism of inverse sequences $\boldsymbol{f}\left(\boldsymbol{j}^{n}\right): \boldsymbol{X} \rightarrow \boldsymbol{Y}$, and thus, it determines (up to homotopy) a mapping $f\left(j^{n}\right): X \rightarrow Y$. Consequently, every $n$-hyperladder $F_{n}=\left(\boldsymbol{f}_{\boldsymbol{j}^{n}}\right) \in \underline{L}_{n}(\boldsymbol{X}, \boldsymbol{Y})$ determines a countable family $\left(\boldsymbol{f}\left(\boldsymbol{j}^{n}\right) \equiv \boldsymbol{f}_{\alpha}\right)$ of morphisms of inverse sequences $\boldsymbol{f}_{\alpha}: \boldsymbol{X} \rightarrow \boldsymbol{Y}$, and thus, it determines a countable family $\left(f_{\alpha}\right)$ of mappings $f_{\alpha}: X \rightarrow Y$ (the chosen $n$ is irrelevant). Further, if $F_{n}=\left(\boldsymbol{f}_{\boldsymbol{j}^{n}}\right) \simeq\left(\boldsymbol{f}_{\boldsymbol{j}^{n}}^{\prime}\right)=F_{n}^{\prime}$, then (by Definition 2.7), for every $\alpha$ there exists an $\alpha^{\prime}$ such that $\boldsymbol{f}_{\alpha^{\prime}} \simeq \boldsymbol{f}_{\alpha^{\prime}}^{\prime}$, and thus, $f_{\alpha^{\prime}} \simeq f_{\alpha^{\prime}}^{\prime}$. Moreover, one can partially order the index set $\boldsymbol{J}(n)$ in an obvious way, such that

$$
(\forall \alpha \in \boldsymbol{J}(n))\left(\exists \alpha^{\prime} \geq \alpha\right)\left(\forall \alpha^{\prime \prime} \geq \alpha^{\prime}\right) f_{\alpha^{\prime \prime}} \simeq f_{\alpha^{\prime \prime}}^{\prime}
$$

Therefore, every $\underline{S}_{n}$-morphism $\boldsymbol{F}_{n} \in \underline{\mathcal{S}}(n)(\boldsymbol{X}, \boldsymbol{Y})$, i.e. $S_{n}$-morphism $\Phi_{n} \in$ $\mathcal{S}(n)(X, Y)$, is a family $\left(\left[f_{\alpha}^{\beta}\right]\right), \alpha \in \boldsymbol{J}(n), \beta \in B$, of the homotopy classes of 
mappings $f_{\alpha}^{\beta}: X \rightarrow Y$ satisfying the following condition:

$$
\left(\forall \beta, \beta^{\prime} \in B\right)(\forall \alpha \in \boldsymbol{J}(n))\left(\exists \alpha^{\prime} \geq \alpha\right)\left(\forall \alpha^{\prime \prime} \geq \alpha^{\prime}\right) f_{\alpha^{\prime \prime}}^{\beta} \simeq f_{\alpha^{\prime \prime}}^{\beta^{\prime}}
$$

Conversely, it is obvious that every family $\left(\left[f_{\alpha}\right]\right), \alpha \in \boldsymbol{J}(n)$, of the homotopy classes of mappings $f_{\alpha}: X \rightarrow Y$ determines an $n$-hyperladder $F_{n}=\left(\boldsymbol{f}_{\boldsymbol{j}^{n}}\right) \in$ $\underline{L}_{n}(\boldsymbol{X}, \boldsymbol{Y})$. (Indeed, let $\boldsymbol{f}_{\boldsymbol{j}^{n}}$ be induced by $\boldsymbol{f}_{\alpha}$ which comes from $f_{\alpha}, \alpha=\boldsymbol{j}^{n}$ !) Consequently, every family $\left(\left[f_{\alpha}^{\beta}\right]\right), \alpha \in \boldsymbol{J}(n), \beta \in B$, of the homotopy classes of mappings $f_{\alpha}^{\beta}: X \rightarrow Y$ satisfying the above condition, determines an $\underline{S}_{n}$ morphism $\boldsymbol{F}_{n} \in \underline{\mathcal{S}}(n)(\boldsymbol{X}, \boldsymbol{Y})$, i.e. $S_{n}$-morphism $\Phi_{n} \in \mathcal{S}(n)(X, Y)$, for each $n$. Recall that, in this case, every shape morphism $[\boldsymbol{f}] \in \underline{\mathcal{S}} h(\boldsymbol{X}, \boldsymbol{Y})$, i.e. shape morphism $\phi \in \mathcal{S h}(X, Y)$, is actually the homotopy class $[f]$ of a mapping $f: X \rightarrow Y$.

In the case of a morphism $\Phi \in \mathcal{S}(\mathbb{N})(X, Y)$, one should notice that $\Phi=$ $\left(\Phi_{n}\right), \Phi_{n} \in \mathcal{S}(n)(X, Y)$, and apply the previous description for every $n \in \mathbb{N}$.

Now, in view of Corollary 3.5, the next corollary arises.

Corollary 6.1. Let $X$ and $Y$ be compact ANR's. Then $X$ and $Y$ are homotopy equivalent if and only if they are isomorphic objects in the category $\mathcal{S}(1)$.

Proof. If $X \simeq Y$ then $S h(X)=S h(Y)$ and thus, by Corollary 3.5 (i), $X \cong Y$ in every category $\mathcal{S}(n)$. Conversely, if $X \cong Y$ in $\mathcal{S}(1)$ then, according to the above description, $X \simeq Y$ holds in straightforward manner. (One may also compare the proof of Theorem 3.4 (i).)

TheOREm 6.2. Let $X$ and $Y$ be FANR's. Then $X$ and $Y$ are of the same shape if and only if they are isomorphic objects in the subcategory $\mathcal{S}_{*}(2) \subseteq$ $\mathcal{S}(2)$. Even more, if $X$ is an FANR and $Y$ is a compactum such that $X \cong Y$ in $\mathcal{S}_{*}(2)$, then $Y$ is an FANR and $\operatorname{Sh}(X)=\operatorname{Sh}(Y)$.

Proof. The necessity holds by Corollary 3.5 (i). To prove the rest of the theorem, it suffices to prove the final assertion. Let $X$ be an FANR and let $Y$ be a compactum such that $X \cong Y$ in the category $\mathcal{S}_{*}(2)$. Then there exists a pair of the uniform 2-hyperladders $F_{2}=\left(f_{2}, \boldsymbol{f}_{\boldsymbol{j}^{2}}\right): \boldsymbol{X} \rightarrow \boldsymbol{Y}$, $G_{2}=\left(g_{2}, \boldsymbol{g}_{\boldsymbol{i}^{2}}\right): \boldsymbol{Y} \rightarrow \boldsymbol{X}$ such that $G_{2} F_{2} \simeq 1_{\boldsymbol{X} 2}$ and $F_{2} G_{2} \simeq 1_{\boldsymbol{Y} 2}$ in the category $\underline{\mathcal{L}}_{0}(2)$, where $\boldsymbol{X}$ and $\boldsymbol{Y}$ are associated with $X$ and $Y$, respectively. By following the proof of Lemma $4.5(n=3)$, one can verify the following fact:

$$
\left(\forall j_{1}\right)\left(\exists i_{1}\right)\left(\forall i_{1}^{\prime} \geq i_{1}\right)\left(\exists j_{1}^{\prime} \geq j_{1}\right)\left(\forall j_{2} \geq j_{1}^{\prime}\right)\left(\exists i_{2} \geq i_{1}^{\prime}\right)\left(\forall i_{2}^{\prime} \geq i_{2}\right)\left(\exists j_{2}^{\prime} \geq j_{2}\right)
$$

and there exist mappings $f_{l}^{3}: X_{i_{l}} \rightarrow Y_{j_{l}}, l=1,2,3$, and $g_{l}^{3}: Y_{j_{l}^{\prime}} \rightarrow X_{i_{l}^{\prime}}$, $l=1,2$, making the following diagram commutative up to homotopy. 


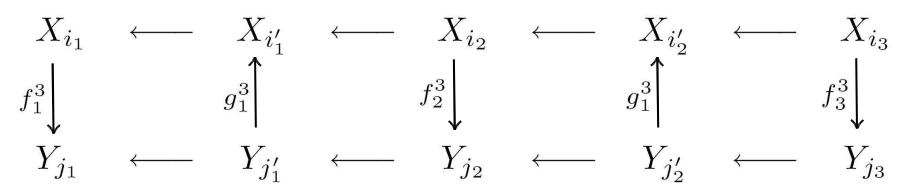

By [6], Theorems 6 (and its proof, $n=3$ ), 7 and $7^{\prime}$ (the assumption that $X$ is a pointed FANR is superfluous), we infer that $Y$ is an FANR and $\operatorname{Sh}(X)=\operatorname{Sh}(Y)$.

\section{REFERENCES}

[1] K. Borsuk, Theory of Shape, Monografie Matematyczne 59, Polish Scientific Publishers, Warszawa, 1975.

[2] K. Borsuk, Some quantitative properties of shapes, Fund. Math. 93 (1976), 197-212.

[3] K. R. Goodearl and T. B. Rushing, Direct limit groups and the Keesling-Mardešić shape fibration, Pacific J. Math. 86 (1980), 471-476.

[4] H. Herlich and G. E. Strecker, Category Theory, Allyn and Bacon Inc., Boston, 1973.

[5] J. Keesling and S. Mardešić, A shape fibration with fibers of different shape, Pacific J. Math. 84 (1979), 319-331.

[6] S. Mardešić, Comparing fibres in a shape fibration, Glasnik Mat. 13(33) (1978), 317333.

[7] S. Mardešić and N. Uglešić, A category whose isomorphisms induce an equivalence relation coarser than shape, Top. Appl. (to appear).

[8] S. Mardešić and J. Segal, Shape Theory, North Holland, Amsterdam, 1982.

[9] N. Uglešić, A note on the Borsuk quasi-equivalence, submitted.

N. Uglešić

University of Split

Department of Mathematics

21000 Split, Teslina 12/III

Croatia

E-mail: uglesic@pmfst.hr

B. Červar

University of Split

Department of Mathematics

21000 Split, Teslina 12/III

Croatia

E-mail: brankoch@pmfst.hr

Received: 5.4.2004.

Revised: 6.7.2004. \& 13.5.2005. 